\title{
Determining Remaining Useful Life of Aging Cables in Nuclear Power Plants - Interim Study FY13
}

KL Simmons

LS Fifield

MP Westman

P Ramuhalli
AF Pardini

JR Tedeschi

AM Jones

September 2013
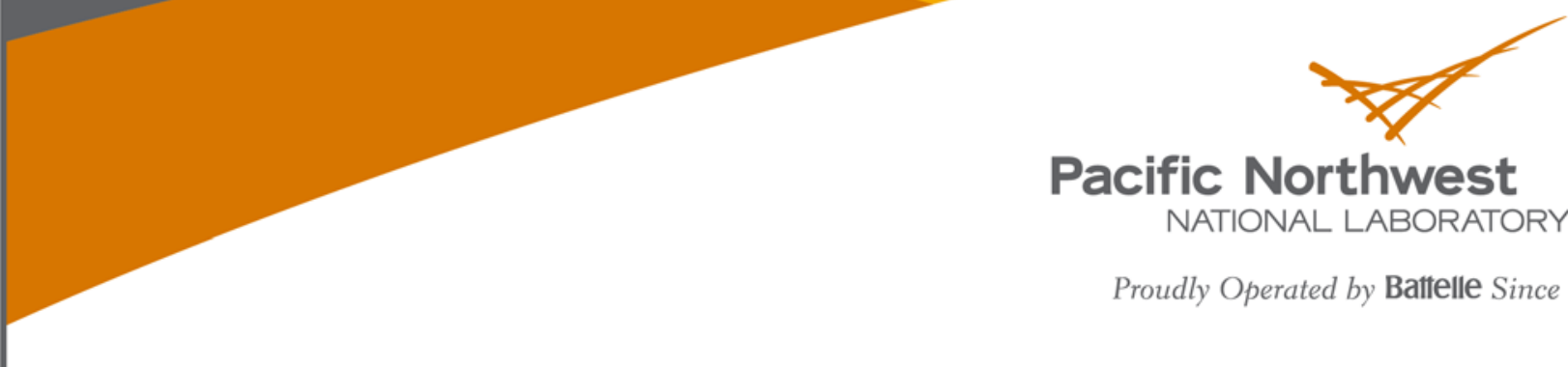

Pacific Northwest

NATIONAL LABORATORY

Proudly Operated by Battelle Since 1965 


\title{
DISCLAIMER
}

This report was prepared as an account of work sponsored by an agency of the United States Government. Neither the United States Government nor any agency thereof, nor Battelle Memorial Institute, nor any of their employees, makes any warranty, express or implied, or assumes any legal liability or responsibility for the accuracy, completeness, or usefulness of any information, apparatus, product, or process disclosed, or represents that its use would not infringe privately owned rights. Reference herein to any specific commercial product, process, or service by trade name, trademark, manufacturer, or otherwise does not necessarily constitute or imply its endorsement, recommendation, or favoring by the United States Government or any agency thereof, or Battelle Memorial Institute. The views and opinions of authors expressed herein do not necessarily state or reflect those of the United States Government or any agency thereof.

\author{
PACIFIC NORTHWEST NATIONAL LABORATORY \\ operated by \\ BATTELLE \\ for the \\ UNITED STATES DEPARTMENT OF ENERGY \\ under Contract DE-AC05-76RL01830
}

Printed in the United States of America
Available to DOE and DOE contractors from the Office of Scientific and Technical Information,
P.O. Box 62, Oak Ridge, TN 37831-0062;
ph: (865) 576-8401
fax: (865) 576-5728
email: reports@adonis.osti.gov

\author{
Available to the public from the National Technical Information Service, \\ U.S. Department of Commerce, 5285 Port Royal Rd., Springfield, VA 22161 \\ ph: (800) 553-6847 \\ fax: (703) 605-6900 \\ email: orders@ntis.fedworld.gov \\ online ordering: http://www.ntis.gov/ordering.htm
}

This document was printed on recycled paper. 


\title{
Determining Remaining Useful Life of Aging Cables in Nuclear Power Plants - Interim Study FY13
}

\author{
KL Simmons \\ AF Pardini \\ LS Fifield \\ JR Tedeschi \\ MP Westman \\ AM Jones \\ P Ramuhalli
}

September 2013

Prepared for

the U.S. Department of Energy

under Contract DE-AC05-76RL01830

Pacific Northwest National Laboratory

Richland, Washington 99352 



\section{Executive Summary}

The motivation for this study stems from the need to address the aging management of incontainment cables at nuclear power plants (NPPs). The most important criterion for cable performance is its ability to withstand a design-basis accident. With nearly $1000 \mathrm{~km}$ of power, control, instrumentation, and other cables typically found in a NPP, it would be a significant undertaking to inspect all of the cables. Degradation of the cable jacket, electrical insulation, and other cable components is a key issue that is likely to affect the ability of the currently installed cables to operate safely and reliably for another 20 to 40 years beyond the initial operating life. The development of one or more nondestructive evaluation (NDE) techniques and supporting models that could assist in determining the remaining life expectancy of cables or their current degradation state would be of significant interest. The ability to nondestructively determine material and electrical properties of cable jackets and insulation without disturbing the cables or connections has been deemed essential.

Currently, the only technique accepted by industry to measure cable elasticity (the gold standard for determining cable insulation degradation) is the indentation measurement. All other NDE techniques are used to find flaws in the cable and do not provide information to determine the current health or life expectancy.

There is no single NDE technique that can satisfy all of the requirements needed for making a lifeexpectancy determination, but a wide range of methods have been evaluated for use in NPPs as part of a continuous evaluation program. The commonly used methods are indentation and visual inspection, but these are only suitable for easily accessible cables. Several NDE methodologies using electrical techniques are in use today for flaw detection but there are none that can predict the life of a cable.

There are, however, several physical and chemical property changes in cable insulation as a result of thermal and radiation damage. In principle, these properties may be targets for advanced NDE methods to provide early warning of aging and degradation. Examples of such key indicators include changes in chemical structure, mechanical modulus, and dielectric permittivity. While some of these indicators are the basis of currently used technologies, there is a need to increase the volume of cable that may be inspected with a single measurement, and if possible, to develop techniques for in-situ inspection (i.e., while the cable is in operation). This is the focus of the present report.

Several approaches to nondestructively measuring key indicators of cable aging and degradation may be available, and could include chemical, mechanical, and electrical measurements. Electrical and acoustic measurements are potential alternative NDE approaches that may be capable of providing in-situ assessments of cable condition and remaining useful life.

Measurement studies were conducted with samples of aged ethylene propylene rubber (EPR) cable (aged at $140^{\circ} \mathrm{C}$ ) using acoustic and electromagnetic methods to determine sound velocity and dielectric permittivity. The complex permittivity results using the high-temperature dielectric probe display promise for measuring a change in the real part of the dielectric constant and relating the change to cable age. 
Using acoustic methods that can provide a direct relation to the elastic modulus of the cable material seems feasible. Preliminary baseline velocity measurements in EPR polymers are in line with published data. However, the acoustic measurement methods need further study to determine if the sound speed (and potentially attenuation) may be an indicator of age that directly correlates with indenter modulus and elongation at break. Further work can be done to optimize the ultrasonic transducers and analysis methods selected as well as provide a more reliable method for positioning and coupling them. Additional studies may also be needed to determine the viability of approaches that directly measure changes in polymer chemistry as a result of aging; these are planned for future phases of research. These studies will use a laboratory-scale test-bed that was assembled at Pacific Northwest National Laboratory and is capable of aging cables and performing measurements in-situ. 


\section{Acronyms and Abbreviations}

$\mathrm{AC}$

AMP

AMS

ATR

CRP

DSC

DOE

$\mathrm{EAB}$

EPDM

EPR

EPRI

FDR

FTIR

GALL

GCMS

IAEA

IR

ISI

LCR

LIRA

LTO

LWRS

MAaD

MW

NDE

NMR

NPP

NRC

OID

PNNL

SSC

TDR

TGA

TS

VNA

XLPE alternating current

aging management program

Analysis and Measurement Services Corporation

attenuated total reflection

coordinated research project

differential scanning calorimeter

U.S. Department of Energy

elongation at break

ethylene propylene diene monomer rubber

ethylene propylene rubber

Electric Power Research Institute

frequency domain reflectometry

Fourier Transform Infrared (Spectroscopy)

Generic Aging Lessons Learned

gas chromatography - mass spectrometry

International Atomic Energy Agency

infrared

in-service inspection

inductance/capacitance/resistance

line resonance analysis

long-term operation

Light Water Reactor Sustainability Program

Materials Aging and Degradation

molecular weight

nondestructive evaluation

nuclear magnetic resonance

nuclear power plant

U.S. Nuclear Regulatory Commission

oxidation induction time

Pacific Northwest National Laboratory

systems, structures, and components

time domain reflectometry

thermogravimetric analysis

tensile strength

vector network analyzer

cross-linked polyethylene 


\section{Contents}

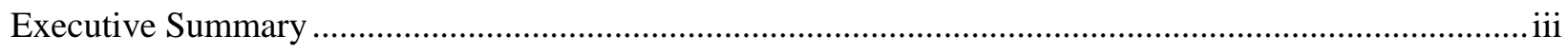

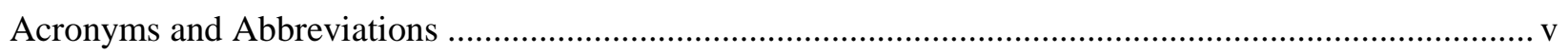

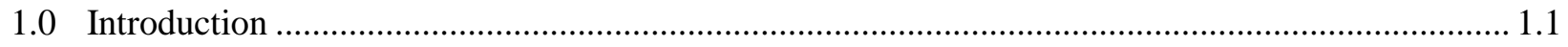

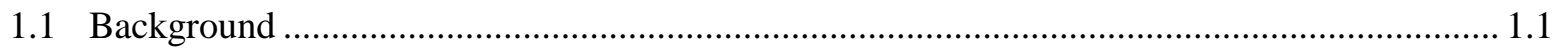

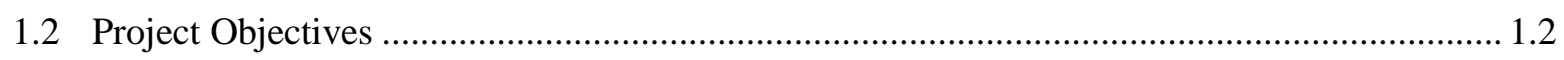

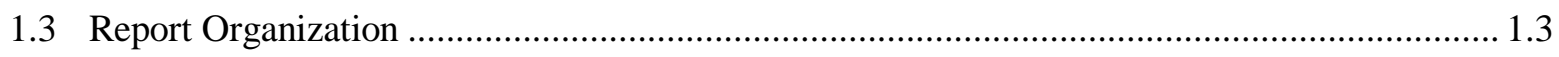

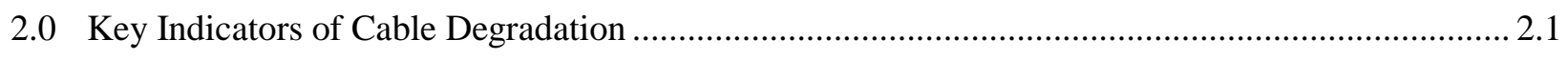

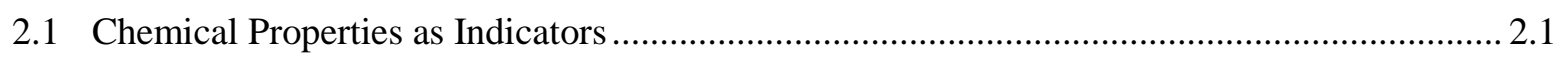

2.2 Physical Properties as Indicators of Chemical Change ......................................................... 2.4

2.2.1 Mechanical Properties as Indicators....................................................................... 2.4

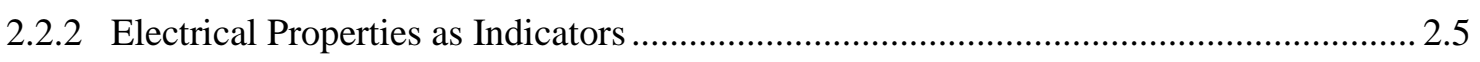

3.0 Experimental Assessment of Key Indicators ........................................................................ 3.1

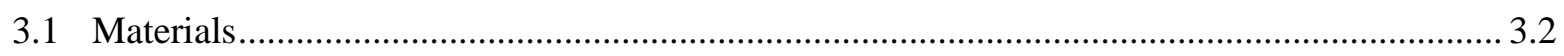

3.2 Measurement Techniques for Chemical Properties............................................................. 3.2

3.2.1 Fourier Transform Infrared Spectroscopy .................................................................. 3.2

3.2.2 Differential Scanning Calorimetry ….................................................................... 3.3

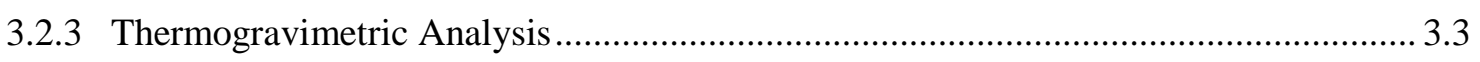

3.3 Measurement Techniques for Mechanical Properties ............................................................ 3.4

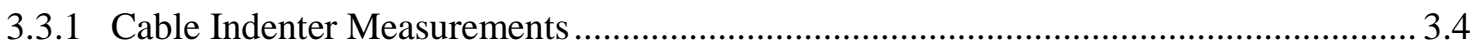

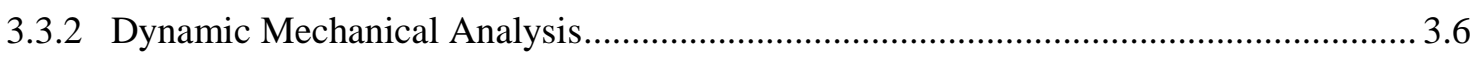

3.3.3 Ultrasonic Measurements of Sound Speed and Attenuation ........................................ 3.9

3.4 Measurement Techniques for Electrical Properties.............................................................. 3.13

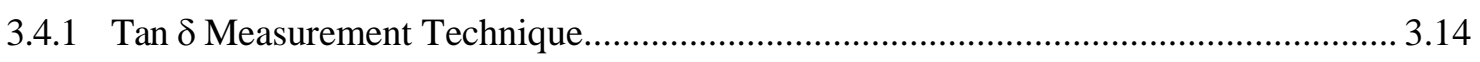

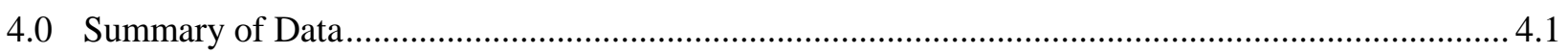

4.1 Acoustic Measurements ................................................................................................. 4.1

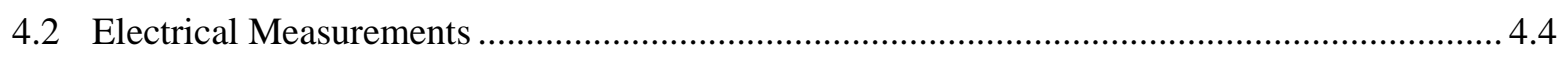

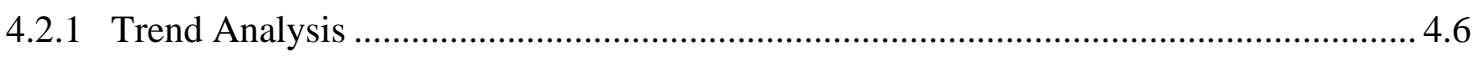

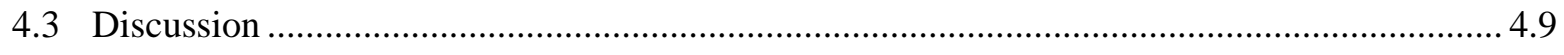

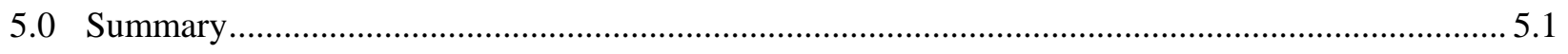

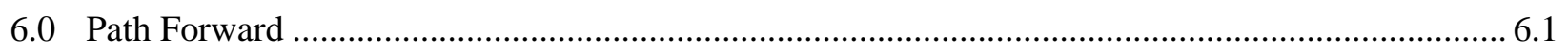

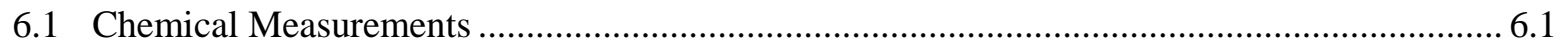

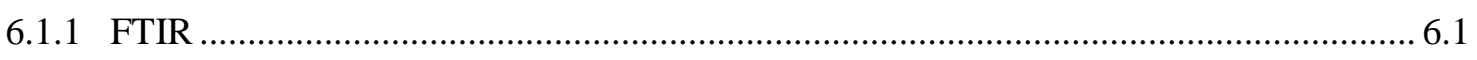

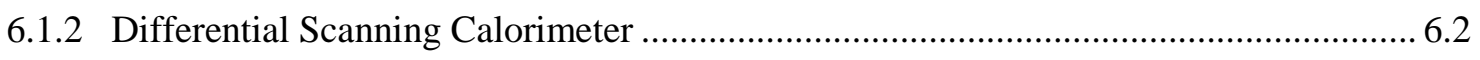

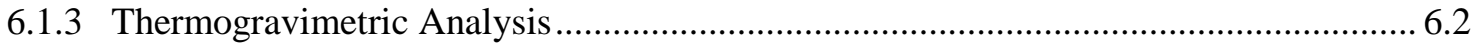

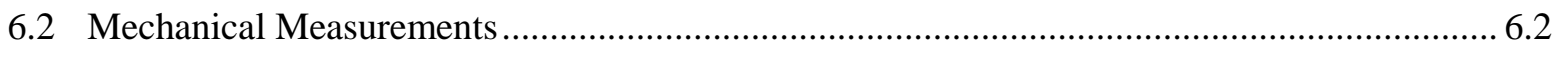

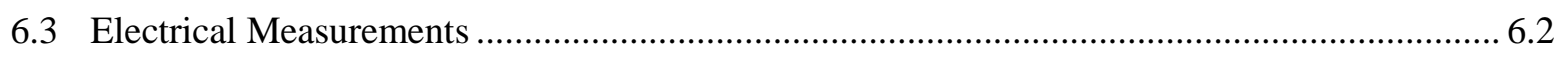




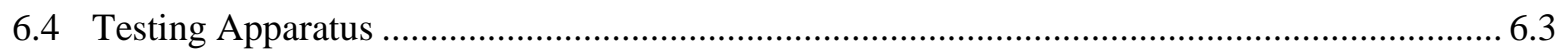

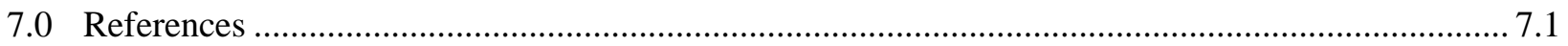

Appendix A - Real Component of the Complex Permittivity Measurements for Aged Cable

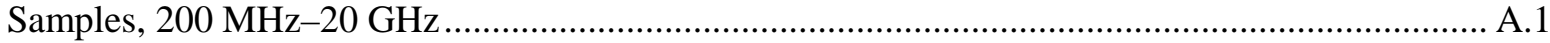




\section{Figures}

1.1 Overview of Research Tasks for Cable Aging Detection and Remaining Life Assessment............ 1.2

3.1 Literature Review on the Frequency of Cable Aging Tests ....................................................... 3.1

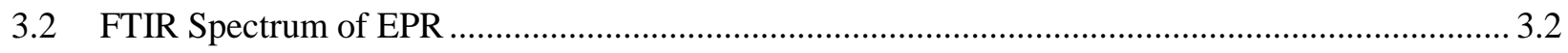

3.3 Illustrative DSC Curve of EPDM Showing Glass Transition, Melting Point and

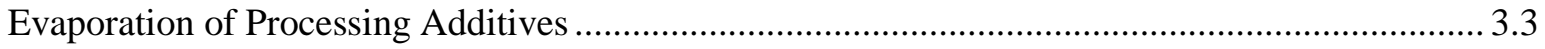

3.4 Illustrative TGA Showing Mass Loss due to Decomposition of Distinct Components................... 3.4

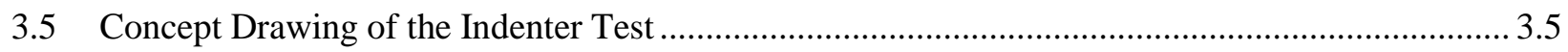

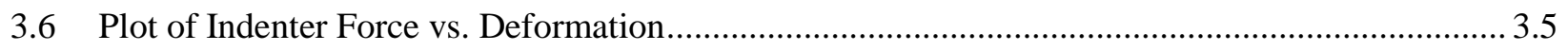

3.7 Illustration of DMA Instrument with Simple Schematic and Input/Output Waveforms for

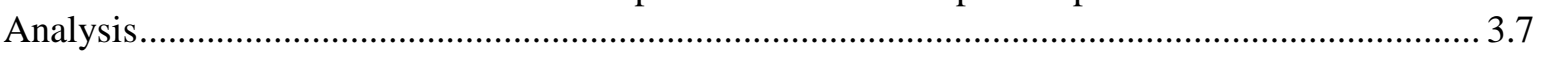

3.8 DMA Data Schematic Illustrating the Available Information Determined by DMA ..................... 3.8

3.9 DMA Data for Unaged Okoguard Jumper Cable ......................................................................... 3.8

3.10 Storage Modulus of $140^{\circ} \mathrm{C}$ Thermally Aged EPR Okoguard Jumper Cable .................................. 3.9

3.11 Ultrasonic Transducer Configuration for Standoff Measurement of EPR Sample ....................... 3.11

3.12 PNNL SNAP Ultrasonic System and PNNL Acoustic Microscopy System................................ 3.12

3.13 Phasor Relationship between the Real and Imaginary Components of Permittivity..................... 3.14

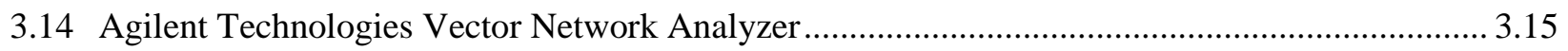

3.15 Steps of Calibration Process................................................................................................... 3.16

3.16 Complex Permittivity Measurements Obtained during Calibration Process................................ 3.16

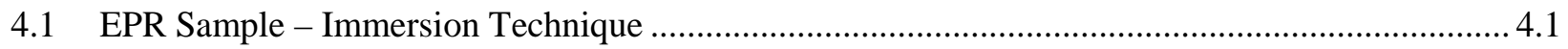

4.2 A-Scan Display from Front and Back Wall of Cable Sample........................................................ 4.2

4.3 UT Signals from the Front and Back Face of the EPR Sample ................................................... 4.3

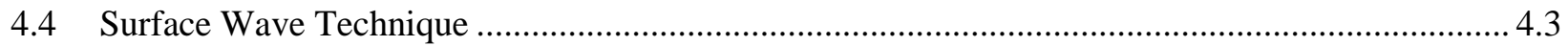

4.5 Initial (No Exposure) Dataset, Real Component of the Complex Permittivity ............................... 4.5

4.6 Sample Exposed to $140^{\circ} \mathrm{C}$ for 1200 Hours, Real Component of the Complex Permittivity............ 4.6

4.7 Minimum $\varepsilon^{\prime}$ Trace Values as a Function of Frequency and Cable Age......................................... 4.7

4.8 Minimum $\varepsilon^{\prime}$ Values at $7.5 \mathrm{GHz}$ vs. Cable Age ........................................................................... 4.8

4.9 Maximum $\varepsilon^{\prime}$ Trace Values as a Function of Frequency and Cable Age ....................................... 4.8

4.10 Maximum $\varepsilon^{\prime}$ Values at 7.5G Hz vs. Cable Age ........................................................................... 4.9

6.1 (a) Thermo Scientific Smart iTR ${ }^{\mathrm{TM}}$ Attenuated Total Reflectance Measurement of EPR Cross-section Slices. Inset: EPR cross-section sliced into layers. (b) FTIR ATR spectrum

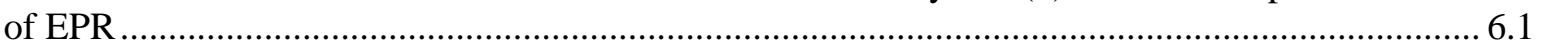

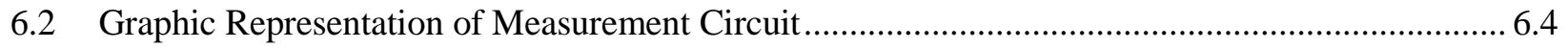

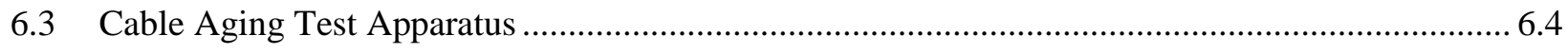




\section{Tables}

2.1 Medium Voltage EPDM Power Cable Insulation Formulation (Vanderbilt 2010)......................... 2.2

2.2 Medium Voltage XLPE Power Cable Insulation Formulation (Vanderbilt 2010).......................... 2.3

3.2 Commercially Available Techniques for Cable Inspection ......................................................... 3.13

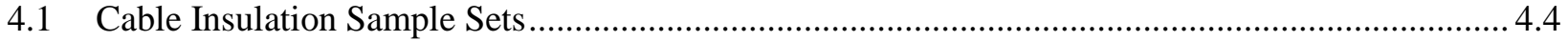




\subsection{Introduction}

This Pacific Northwest National Laboratory (PNNL) interim report describes progress to date on investigating aging conditions that provide key indicators of cable aging and identifying measurement technologies that may be used as potential methods for examining these cables. It is submitted in fulfillment of deliverable "Complete and deliver report on measurements of physical properties on cables subjected to range of accelerated aging conditions, and assess result for key early indicators of cable aging” (Level M3 Milestone).

\subsection{Background}

In July 2012, a workshop (Simmons et al. 2012) was held to lay the groundwork for a research and development roadmap to address aging cable management in NPPs. This workshop brought together subject matter experts from the U.S. Nuclear Regulatory Commission (NRC), U.S. Department of Energy (DOE) national laboratories, the Electric Power Research Institute (EPRI), universities, and cable manufacturers and inspectors.

The workshop focused on identifying changes in chemical structure that would be a precursor to eventual failure of an aging cable and the current state-of-the-art in nondestructive evaluation (NDE) methods that could be applied to estimate the remaining life of the cable. These changes in chemical structure are most likely to be caused by the environment the cable is in (thermal, radiation, moisture, chemicals) and the mechanical load (both static and dynamic) that is being applied. Therefore, the development of new NDE methods or development of new techniques using existing NDE methods is of significant interest. The ability to perform a nondestructive test to determine chemical, physical, mechanical, and electrical properties of the cable jackets and insulation without significant disturbance of the cables and connectors as they lay in-situ is essential.

There have been many programs and years of research to address the problems of aging cables with no single NDE method identified that can satisfy all of the requirements needed to assess life expectancy. The most common methods used are visual (looking for cracking and discoloration indicative of cable aging) and a method that indents the surface of the cable jacket (measures cable elasticity and correlates to cable aging). These are essentially the only methods currently acceptable to industry. All other current methods (such as time and frequency domain analysis, inductance and capacitance measurements, Tan $\delta$, etc.) provide flaw detection, but none can predict the expected life of the cable.

The workshop identified three important areas that should be considered to assess overall cable aging:

1. Determination of the key chemical, physical and electrical indicators of cable aging

2. Advance current and develop new NDE methods to enable in-situ cable condition assessment

3. Develop models to assist in predicting remaining useful life of aging cables.

Figure 1.1 succinctly illustrates the importance of using NDE to predict remaining useful life of aging cables and the individual properties that must be considered. 


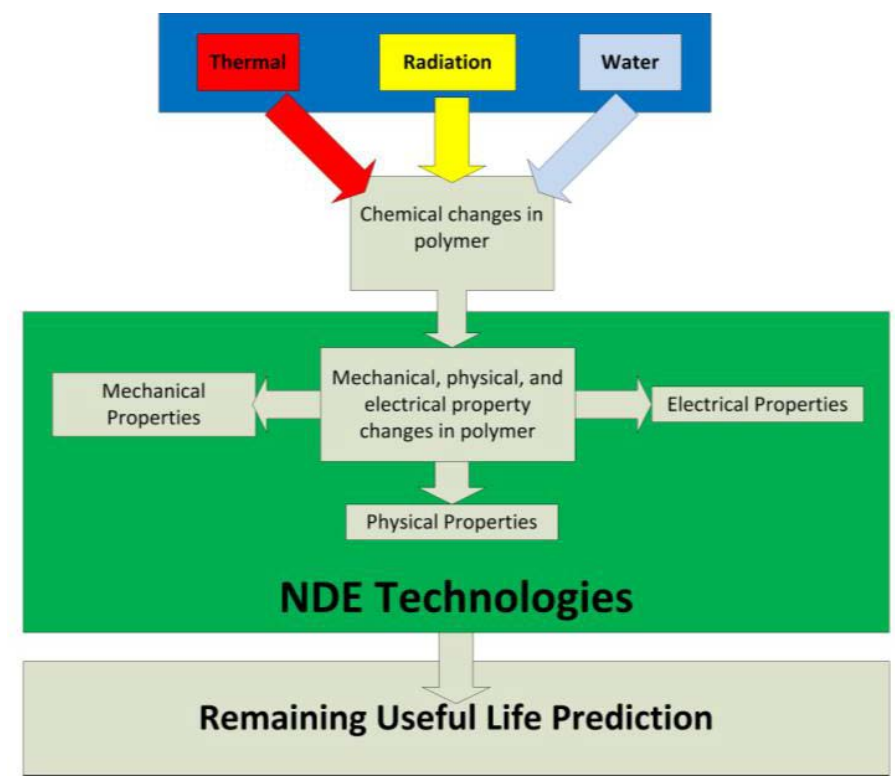

Figure 1.1. Overview of Research Tasks for Cable Aging Detection and Remaining Life Assessment

\subsection{Project Objectives}

The overall objectives of the project are to develop the technical basis for assessing the level and impact of cable aging and degradation in nuclear power plants. The project addresses the overall gaps that were identified at the workshop in FY2012 using a phased approach. This phased approach will address the three areas identified from the workshop:

1. Determination of the key indicators of cable aging.

2. Advance current, and develop new, NDE methods by using insights from the determination of key indicators.

3. Develop models that use the advances in key indicators and NDE methods to assist in predicting remaining life of cables.

The focus of the project thus far has been on measurements of physical properties that act as key indicators of aging of ethylene propylene rubber (EPR)-insulated cable, which is one of the primary cable types used in nuclear power plants (NPPs). The overall objective of this effort is to complete an assessment of measurements of physical properties on cables subjected to a range of accelerated aging conditions, and assess results for key early indicators of cable aging. The initial assessment evaluated available literature in current advances in polymer science to determine likely measurable conditions that can serve as key indicators of cable aging. In parallel, NDE measurement methods sensitive to these conditions as well as NDE methods currently being considered for cable aging assessment were identified to provide a foundation for further investigation. The assessment is continuing, and the information presented in the following sections is based on literature evaluated to date. Follow-on work will continue to evaluate the potential of determining cable remaining useful life using the identified key indicators. To assist in this effort, we will continue to identify cables that can be (or have been) subjected to aging, 
measure physical properties of these aged cables using existing or possibly new NDE methods, and document results in a future technical report.

This report summarizes our findings to date and observations from the available information and the collection of experimental data.

\subsection{Report Organization}

This document is organized as follows. Section 2 discusses the measurement needs from a materials science perspective. Specifically, the impact of degradation mechanisms of concern on materials microstructure, and the key measurements that are needed for assessment of impact on structural integrity are summarized. Section 3 summarizes the leading candidates for nondestructive measurements that may be applicable to the problem at hand. Section 4 summarizes the experimental data collected to date and describes the initial results of analysis of this data. Sections 5 and 6 summarize the findings to date and discuss the path forward. In addition, appendices are included that provide details of the data from the measurement of key electrical indicators. 



\subsection{Key Indicators of Cable Degradation}

The stressors in fielded polymers — such as heat, radiation, and moisture-modify polymer chemistry and result in changes in mechanical, physical, and electrical performance of the materials. The nuclear industry uses a variety of polymers and elastomers for insulators and jacketing materials with several of these materials often used in combination. The industry has accepted indentation modulus as a key indicator and elongation at break (EAB) as a measure of cable remaining useful life. EPRI Technical Report 1008211, “Initial Acceptance Criteria Concepts and Data for Assessing Longevity of Low-Voltage Cable Insulations and Jackets” (EPRI 2005) develops a basis for acceptance criteria and evaluates the aging profiles for many commonly used cable jackets and polymers. The report describes 50 percent $\mathrm{EAB}$ as a conservative practical end-of-life threshold for cables that may be stressed during maintenance or subjected to loss-of-coolant accident (LOCA) exposure. The report also discusses the basis for cautious continued use of cables beyond the 50 percent EAB threshold.

While these methods are broadly accepted in industry, the indentation modulus is a localized measure of cable insulation degradation that has been correlated to EAB. Measurements of modulus from one location cannot be readily extrapolated to assessing the condition of the cable over longer sections, especially in sections that are inaccessible and may experience different environmental conditions. EAB, on the other hand, is an ex-situ measure of cable remaining useful life. Developing approaches for NDE that overcome the potential limitations of these methods will require a fundamental assessment of NDE methods for their applicability to cable remaining useful life estimation.

The key to success in using nondestructive evaluation (NDE) methods for this project will be to determine what chemical changes occur that affect the properties of the insulator or jacketing materials that could lead to determining the remaining useful life based on these chemical changes. EPRI document TR-103841, "Low-Voltage Environmentally-Qualified Cable License Renewal Industry Report; Revision 1” (EPRI 1994) provides a technical basis for license renewal for low-voltage environmentally-qualified cable. Specifically, the evaluation discusses age-related degradation mechanisms, the effects of age-related degradation on functionality of equipment, and aging management options. The nuclear power industry has considered age-related degradation mechanisms, but does not appear to have correlated these with NDE detection methods. The industry also has a Class $1 \mathrm{E}$ qualification test that cables must be subjected to and that is in accordance with IEEE standards 323-2003 and 383-2003.

It is important to first understand the aging mechanisms chemically. Based on the polymer system being investigated, it is important to determine the composition of the materials involved. Questions that will need to be addressed include: Are there polymer additives present that were included to aid in processing or to limit thermal degradation? Are there plasticizers included for flexibility? How do they affect the aging properties of the system? Is the integrity of the backbone of the polymer the key indicator to material property changes with aging? What NDE methods can be used to indicate polymer changes independent of whether the polymer is amorphous or semi-crystalline?

\subsection{Chemical Properties as Indicators}

The chemical composition of cable insulation compounds varies among cables manufacturers with their strategies to meet or exceed IEEE qualification specifications. Table 2.1 illustrates a medium- 
voltage cable insulating compound with the various additives to the base ethylene propylene diene rubber (EPDM) elastomer. Each of the additives in this example imparts specific properties to the insulating compound. The EPDM/EPR base polymer can have variations in the ethylene content, which affect the level of crystallinity and the glass transition temperature. Higher ethylene rubbers have better oxidative stability and resistance to chain scission during free radical crosslinking. Fillers such as calcined clay and silica will increase the modulus and provide resistance to elongation. The adhesion of the fillers to the elastomer affects the resistance to elongation and any loss of this adhesion over time can alter that resistance. Clay fillers can also retard the cure and therefore require the use of accelerators in the compound. The clay fillers also use silane-treated surfaces for adhesion promotion and to help reduce retardation of the cure. Another type of key ingredient is antioxidants. Antioxidants help with high process temperatures and long-term aging conditions. Other materials such as zinc oxide, low-density polyethylenes, paraffinic waxes, and oils are added as processing aids and plasticizers to enhance physical properties, improve heat resistance, and low-temperature flexibility (Vanderbilt 2010).

Table 2.1. Medium Voltage EPDM Power Cable Insulation Formulation (Vanderbilt 2010)

\begin{tabular}{lc}
\hline \multicolumn{1}{c}{ Ingredients } & $\begin{array}{c}\text { Parts per } \\
\text { Hundred }\end{array}$ \\
\hline Vistalon EPDM 1703P & 100 \\
Translink 37 Calcined Clay & 60 \\
AGERITE MA Antioxidant & 1.5 \\
Drimix A-172 & 1 \\
Zinc Oxide & 5 \\
Lead Red ERD-90 & 5 \\
LDPE Escorene LD-400 & 5 \\
Paraffin Wax (133F M.P.) & 5 \\
VAROX DCP-40KE Peroxide & 6.5 \\
\hline \multicolumn{1}{c}{ Total } & 189 \\
\hline
\end{tabular}

The mechanical properties of polymers are largely dictated by the molecular structure of the polymer backbone. The super-molecular structures of polymers used in cables and jackets can be amorphous or semi-crystalline. Semi-crystalline polymers have randomly oriented crystalline regions with amorphous regions linking them together. It is not uncommon for such polymer systems to include antioxidants, plasticizers, inorganic fillers, and various other additives to enhance the properties of the neat polymer.

The chemical properties of polymer systems, including material composition, are directly related to polymer mechanical, physical, and electrical properties. Any changes to the material composition have a related effect on these other properties. The most common change to the polymer backbone from environmental degradation is chain scission - the cleaving of a one polymer molecule into two pieces. Polymer chain damage can occur in three different ways: (1) chain scission at the ends of the polymer backbone, (2) chain scission randomly along the chain, and (3) chain scission of side pendants from the main backbone. Chain scission decreases polymer molecular weight and affects various other properties such as mechanical (strength and/or modulus), physical (density, glass transition temperature), and electrical (dielectric) performance. The free radicals responsible for chain scission can also lead to the formation of new bonds in the materials, including cross-linking between polymer chains that further alter material properties. 
Gillen et al. (2005) have shown that during radiation aging, reactive species such as radicals are generated uniformly throughout both crystalline and amorphous regions. At temperatures well below the crystalline melting point, these species are trapped in the crystalline regions and are unable to react to form oxidative products because of low chain mobility and low oxygen diffusion rate. Degradation then proceeds primarily through oxidative scission reactions in the amorphous regions, where both chain mobility and oxygen diffusion rates are higher. Because the amorphous regions bind together and produce load transfer between the crystalline blocks, chain scission in these regions has a marked effect on the mechanical properties of the system.

If radiation aging occurs at slightly higher temperatures, nearer the melting region for the crystalline portion, then chain mobility is high enough for the trapped species to react to form covalent chemical cross-links rather than chain scission. In addition, the enhanced mobility enables some recrystallization to occur, which can reform molecular bonds that were broken by oxidative scission in the amorphous regions. The combination of these effects is to effectively 'heal' some of the damage that was created by radiation aging. The overall macroscopic effect is a reduced rate of radiation degradation at higher temperatures.

Exposure to environmental stressors can lead to changes in polymer chemical properties including loss of molecular weight, cross-linking (or gelling), release of volatiles (including additives and plasticizers), functional group transformation (such as carbonyl formation), and polymer backbone conjugation (Scheirs 2000).

Cross-linked polyethylene insulation materials represent completely different chemical compositions than the ethylene propylene rubber (EPR) or EPDM systems. Table 2.2 illustrates the shorter list of chemical compounds that make up a typical cross-linked polyethylene (XLPE) formulation. The peroxide additive to polyethylene decomposes by heat addition just after the polyethylene extrusion process to produce radicals that cross-link the material. Cross-linking provides for much higher temperature resistance by increasing polymer molecular weight. This process significantly increases the resistance to thermal deformation and increases the temperature rating of the cable. Cross-linking also increases material toughness and the resistance of the material to solvents and environmental stress cracking. Antioxidants are added to capture any highly reactive free radicals and improve water-treeing resistance during the life of the cable.

Table 2.2. Medium Voltage XLPE Power Cable Insulation Formulation (Vanderbilt 2010)

\begin{tabular}{lc}
\hline \multicolumn{1}{c}{ Ingredients } & Weight, \% \\
\hline Low Density Polyethylene & $97-98$ \\
VAROX DCP Peroxide & $1.5-2.0$ \\
Antioxidant & $0.2-0.5$ \\
\hline
\end{tabular}

Physical, chemical, and electrical degradation are the key aging mechanisms affecting the XLPE insulation. XLPE materials are an example of materials that can continue to cure over many years to achieve their final crystal formation. Mechanical stresses can also generate free radicals, bond formations, and carbonyl groups in XLPE over time (Crine 2005). Small partial electrical discharges can also degrade the polymer structure. 


\subsection{Physical Properties as Indicators of Chemical Change}

Polymer chains are broadly classified into four key structures: (1) linear, (2) branched, (3) crosslinked, and (4) networked. These chemical structural characteristics directly correlate to many properties of the polymer, most notably mechanical and electrical properties. The ability of chain segments to rotate is influenced by the applied stresses or thermal vibrations that influence the mechanical and thermal characteristics of the polymer. The outcome of these properties can be influenced by any damage to the structure that can change the property relationship. The structure can change the molecular weight, crosslink density, the degree of crystallinity, the chemical resistance, and the electrical properties (Crawford 1998).

Initial literature review has revealed reports of physical property changes in polymers that indicate chemical change and may be correlated to cable and jacket functional life status. Molecular weight is one of the most influential factors of polymer properties. Reduction or increase in effective molecular weight of a polymer system directly affects the energy required to break and form non-covalent bonds between polymer molecules. Thus, the transition between polymer phases is a strong function of polymer chain length. Changes in the melting, glass transition, and gel temperatures of a polymer are measures of scission or cross-linking of the polymer chains. Alteration of polymer average chain length, chain length distribution, and chemical side-group connectivity affect the free volume within the polymer system. Consequently, the macroscopic density of the polymer sample and properties such as gas permeation and liquid (e.g., oil) uptake can be correlated with polymer aging. Other measureable physical property changes as a function of polymer environmental degradation include discoloration, change in refractive index, and change in sound velocity within the material (Crawford 1998).

Material density changes as a function of thermal degradation have been shown to track well with $\mathrm{EAB}$. It was found that the density changes were sensitive enough to track the slow to moderate changes during the oxidation induction period and then increase dramatically. Combining density behavior with modulus changes offers an opportunity for exploiting these two measurements as key indicators for aging (Gillen et al. 1999).

\subsubsection{Mechanical Properties as Indicators}

The large number of chemical and structural characteristics of polymeric materials influences the properties and behavior of the materials over time. The density, stiffness, strength and ductility are influenced by degree of crystallinity in semi-crystalline polymers. The stiffness of rubbers and crosslinked polymers is related to degree of cross-linking, loss of plasticizers, fillers. Melting point and glass transition temperatures are also related to polymer chemical state (Crawford 1998).

The macromolecular polymer chain structure significantly varies from polymer to polymer and the chain arrangement has a large influence on the mechanical properties of the polymer. Chains that are coiled or have a large amount of kinks in them have a tendency to have more flexibility or have high elongation. Long chain structures tend to kinks, coils, and bending that will entangle and intertwine within and between chains in the bulk. This molecular entanglement is an important characteristic of polymers and elastomers and is a key contributor to high elongation, moduli, and strength properties such as those found in rubbers. Likewise with semi-crystalline polymers, highly ordered structures pack 
themselves into organized units with amorphous linking regions between them (Crawford 1998; Vanderbilt 2010).

Key indicators of these changes are noticed in current state-of-the-art technologies such as the indenter modulus that is correlated to the elongation at break. All of these properties are related to changes in polymer structure. Other influenced mechanical properties are creep, recovery and relaxation time that are related to ability of a stressed polymer to recover over time. Mechanical property modifiers such as plasticizers, processing aids and other additives can be altered by loss or consumption of these additives over time. For example, a small loss of plasticizer can significantly alter the elongation and modulus of the material.

Tensile testing has historically been used in the cable industry to evaluate the thermal aging behavior of cables with the primary focus on ultimate tensile elongation, also known as elongation at break (EAB). The EAB is used as a preferred reference metric because it decreases directly with age, while quantities such as tensile strength may increase with age and then start decreasing.

EPRI developed the cable indenter test and correlated experimental EAB to the indenter modulus at a specific temperature. The indenter modulus is one of the standards used by the industry for NDE inspections. The limitation with the indenter modulus technique is that it only allows for measurements in accessible cable areas.

\subsubsection{Electrical Properties as Indicators}

As electrical conductivity is proportional to the product of charge mobility and charge carrier concentration, the conductivity of polymers used in cable and jacket insulation varies with change in charge carrier density in the material. As cables age, multiple processes combine to increase the number of carriers in the polymer insulation material, thereby increasing the conduction and ultimately leading to dielectric breakdown. Increase in the ionic (charge-bearing) content of polymer material over time may result from ingress of moisture or other impurities, departure of additives initially present in the material, creation of charged moieties through bond scission from oxidation or photo-cleavage, or infiltration of surface charges within the material. In addition, change in spatial distribution of heterogeneities in additives, plasticizers and fillers, and rearrangement of polymer structure such as evolution of crystalline phases over time can alter material electrical properties. Dielectric strength (breakdown voltage divided by thickness), insulation resistance (applied voltage divided by leakage current over a given period of time), and electrical conductivity may provide useful measures of the state of change of the material composition of an aging polymer. This state of change may be correlated with other measureable properties of the polymer such as elongation at break, and tied to the remaining functional lifetime of the cable insulation or jacket material (Grellmann and Seidler 2007). 



\subsection{Experimental Assessment of Key Indicators}

Prior literature was reviewed to identify specific properties (physical, chemical, and electrical) of cable insulators that have the potential to serve as key indicators of aging-related degradation. A summary view (Figure 3.1) shows a number of potential approaches to measurement, where the underlying property shows promising trends relative to age of the cable.

Based on this survey, we selected a number of properties for initial assessment. The selection was based on sensitivity of measurement techniques to the property, ease of obtaining measurements, potential for in-situ measurement, potential for developing measurement techniques that can interrogate the cable condition over longer distances, and availability of measurement equipment. This section describes the measurement techniques that are being evaluated experimentally for their sensitivity and reliability during this phase of the assessment. Additional properties (and related measurement techniques) will be evaluated in subsequent phases.

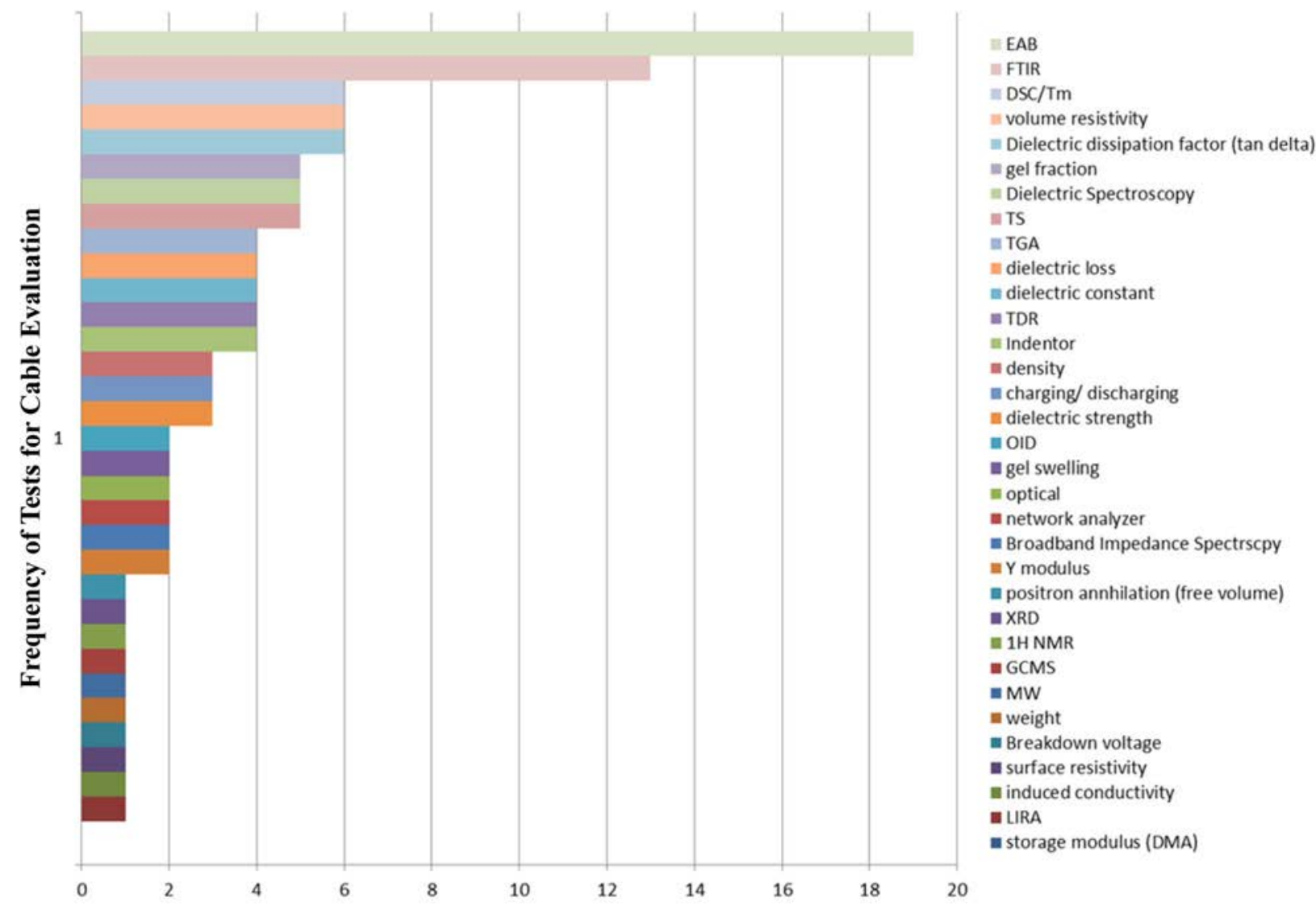

Figure 3.1. Literature Review on the Frequency of Cable Aging Tests 


\subsection{Materials}

The outer sheath material of the cable used in this study is an ethylene propylene-based rubber material, typically referred to as EPR. This material has the registered trade name of Okoguard and is produced by the Okonite Company. The insulation was cut from the cable into specimens of $\sim 5.5$-mm thickness and thermally aged. Samples were heated in a forced air oven at $140^{\circ} \mathrm{C}$. Samples were removed at different time points up to 1200 hours. EAB measurements related the heating time to sample aging down to $~ 50 \%$ absolute EAB representing end of EPR functional life.

\subsection{Measurement Techniques for Chemical Properties}

Chemical changes that polymers undergo with aging include oxidation, cross-linking, and chain scission. Polymer mechanical and electrical properties change with the chemical properties of polymer systems. As a result, analytical techniques are needed to determine the changes in chemistry to better form correlations with mechanical and electrical properties. Commonly used analytical techniques for chemical property changes include Fourier transform infrared spectroscopy (FTIR), differential scanning calorimetry, and thermogravimetric analysis.

\subsubsection{Fourier Transform Infrared Spectroscopy}

Oxidation and crosslinking of cable insulation polymers such as EPR and XLPE inherently introduce new chemical bonds within the material, including $\mathrm{C}=\mathrm{O}$ carbonyl and $\mathrm{C}=\mathrm{C}$ carbon bonds, that have unique vibrational frequencies. A convenient method, therefore, for characterization of related polymer degradation is FTIR spectroscopy. An example FTIR spectrum of EPR is provided in Figure 3.2.

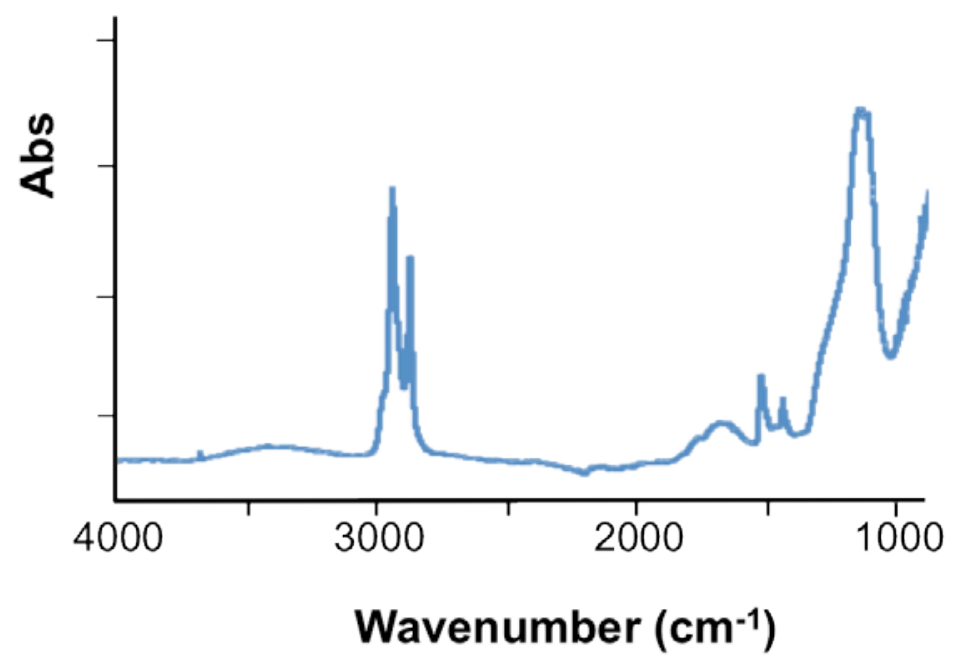

Figure 3.2. FTIR Spectrum of EPR 


\subsubsection{Differential Scanning Calorimetry}

Differential scanning calorimetry (DSC) is a technique that is especially useful for characterization of semi-crystalline polymer systems. DSC measures the flow of heat in and out of test samples over time as a function of sample temperature. Features in a DSC curve include phase change transitions. As illustrated in the DSC curve of EPDM in Figure 3.3, heat flows into a sample with rising temperature to effect endothermic transitions including transition from solid to a glassy state, the glass transition, and from a glassy solid to a melted liquid. Heat is also consumed in the evaporation of volatile compounds such as added processing aids. In a semi-crystalline polymer, it is the material in the crystalline regions that undergoes a distinct melting transition. The integral of the melting peak in the DSC curve is thus a direct measure of the crystalline content of the system. The shape of the DSC curve, including the location of glass transition, is also related to chain scission and cross-linking that the polymer may have experienced.

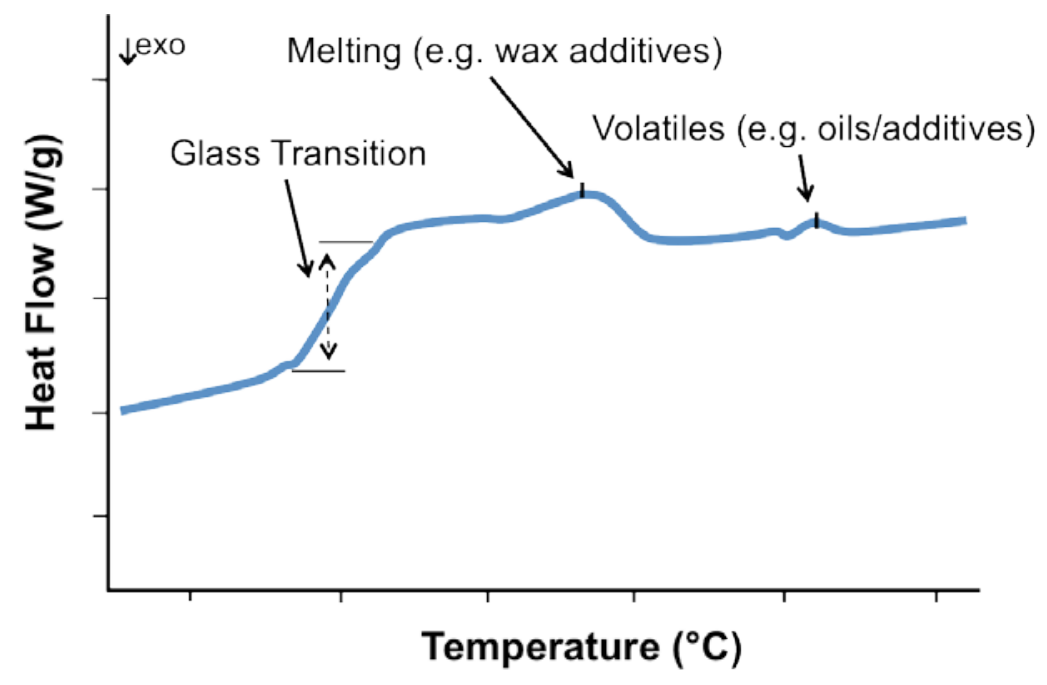

Figure 3.3. Illustrative DSC Curve of EPDM Showing Glass Transition, Melting Point and Evaporation of Processing Additives

\subsubsection{Thermogravimetric Analysis}

In thermogravimetric analysis (TGA), the mass of a sample is monitored as a function of temperature and time. The experiment may be performed under inert, reactive, or oxidizing atmosphere and the TGA may be combined with a mass spectrometer to detect mass fragments of species volatilized during sample heating. Mass loss with heating can reveal copolymer ratio, moisture content, volatile additive content, and inorganic filler content. The decomposition behavior of polymer samples at higher temperatures can also reveal information regarding the extent of chain scission and cross-linking in the polymer. Thermal decomposition in the TGA experiment may be a useful measure of relative degradation and history of polymer samples. The thermogram curve in Figure 3.4 illustrates transitions with 1) onsets of mass loss of distinct constituents, 2) inflections of mass loss curve, and 3) conclusion of mass loss. 


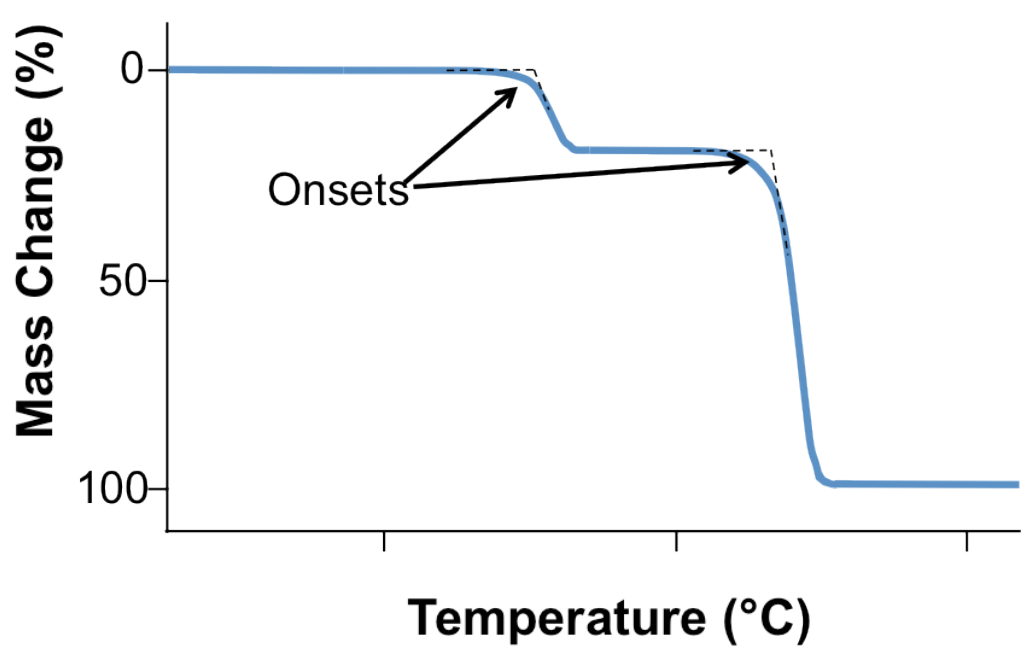

Figure 3.4. Illustrative TGA Showing Mass Loss due to Decomposition of Distinct Components

\subsection{Measurement Techniques for Mechanical Properties}

The most common measurement for sensitivity to aged conditions is destructive tensile testing known as elongation at break. Tensile strength may increase during aging and then dramatically decrease with some insulators. However useful in research, it is not practical to destructively test in-use cables for determining their aged condition (Gillen et al. 1999).

One of the key indicators that has previously been discussed (Yamamoto and Minakawa 2009) is the change in modulus of elasticity of the outer sheath material of a nuclear-grade cable. Aging of polymers used as cable insulation and jacket materials typically causes them to harden, thereby changing their elastic modulus.

Several approaches to measuring elastic modulus (or quantities that are related to the modulus) are possible. The industry standard for measuring modulus is the indenter method, although other approaches that may provide greater sensitivity to changes in modulus are available. These are described in greater detail below.

\subsubsection{Cable Indenter Measurements}

The most prevalent measurement currently used to assess the age of a cable in the field is the "indenter" method (IAEA 2012). This method, shown graphically in Figure 3.5, uses a small-diameter probe to press against a cable. The force needed to compress the EPR jacket to a limited, defined extent is measured. The force $F$ used and the displacement $X$ are plotted against each other as shown in Figure 3.6. The indenter modulus $\sigma$ of the material is the slope of the line relating the change in force $\Delta F$ to the change in displacement $\Delta X$ :

$$
\sigma=\frac{\Delta F}{\Delta X}
$$




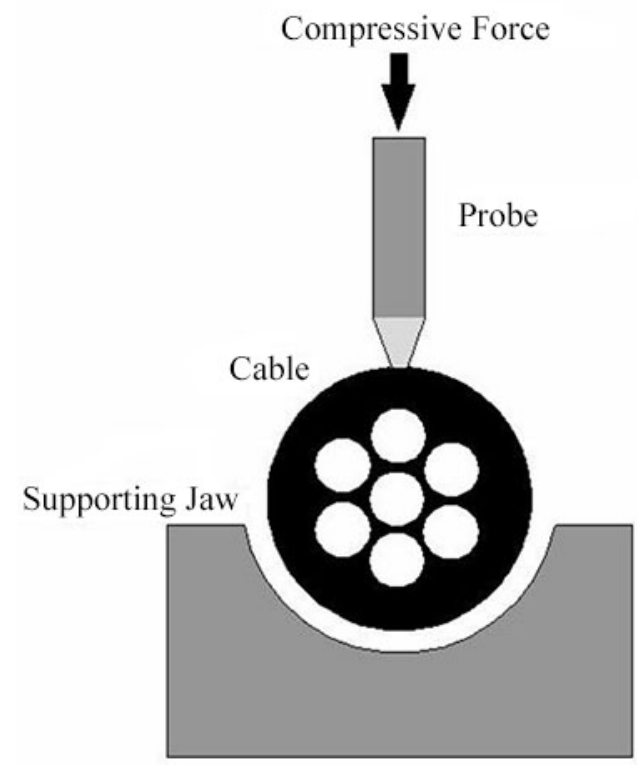

Figure 3.5. Concept Drawing of the Indenter Test

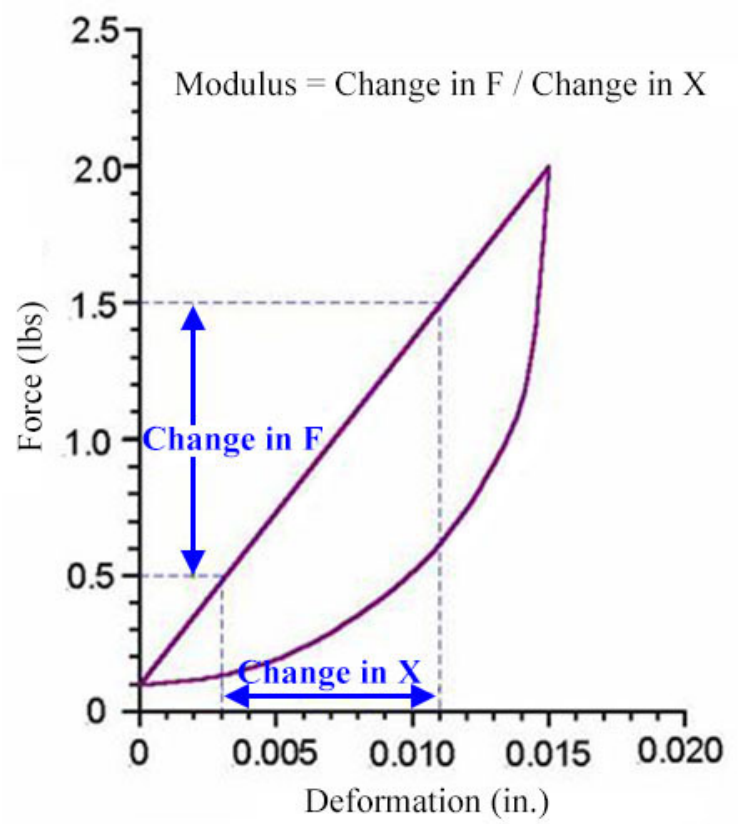

Figure 3.6. Plot of Indenter Force vs. Deformation

This modulus is an indicator of cable aging. It can be plotted at each point in time when a measurement is taken, thereby providing a representation of cable aging over time. Studies indicate that the elastic modulus generally increases with thermal aging (Lofaro et al. 2001). 


\subsubsection{Dynamic Mechanical Analysis}

Pacific Northwest National Laboratory (PNNL) used an advanced measurement system from TA Instruments and a technique known as dynamic mechanical analysis (DMA). DMA measurements are sensitive to polymer transitions, levels of crystallinity, chain scission, and crosslinking (Sepe 1998). The DMA measures the material response to the stimulated stressors applied. The stressors can be temperature, frequency, applied stress, or applied strain, and the DMA can be used to characterize the material's properties as a function of temperature, time, frequency, stress, and atmosphere. The DMA can also be used in thermomechanical analysis mode that can simulate not only the indenter test, but also look at mechanical stressors such as creep and stress relaxation.

The DMA measurements typically apply a sinusoidal force at a 1-Hz frequency while sweeping temperature from $-150^{\circ} \mathrm{C}$ to $150^{\circ} \mathrm{C}$ or higher at $2^{\circ} \mathrm{C} / \mathrm{min}$. The probe tip can have various geometries depending on the stress level of interest. PNNL has used a 0.91-mm probe diameter with a right cylinder geometry. Cross-section samples of $\sim 5.5$-mm thick and $\sim 0.25$-in. wide were cut from aged tensile specimens and subjected to DMA measurement conditions.

Figure 3.7 illustrates how the system is configured to measure the material properties. The oscillating probe with its applied stress and measured strain compares the lag between the applied and measured conditions to calculate storage and loss moduli. The storage and loss moduli are the stored (elastic inphase) and dampened (viscous out-of-phase) energy components, respectively. The ratio of the loss to stored moduli, a measurement of energy dissipation in the material, is the Tan $\delta$. The Tan $\delta$ is analogous to that of the electrical property measurements of which the ratio is a measurement of the lag between the applied and measured loads. 

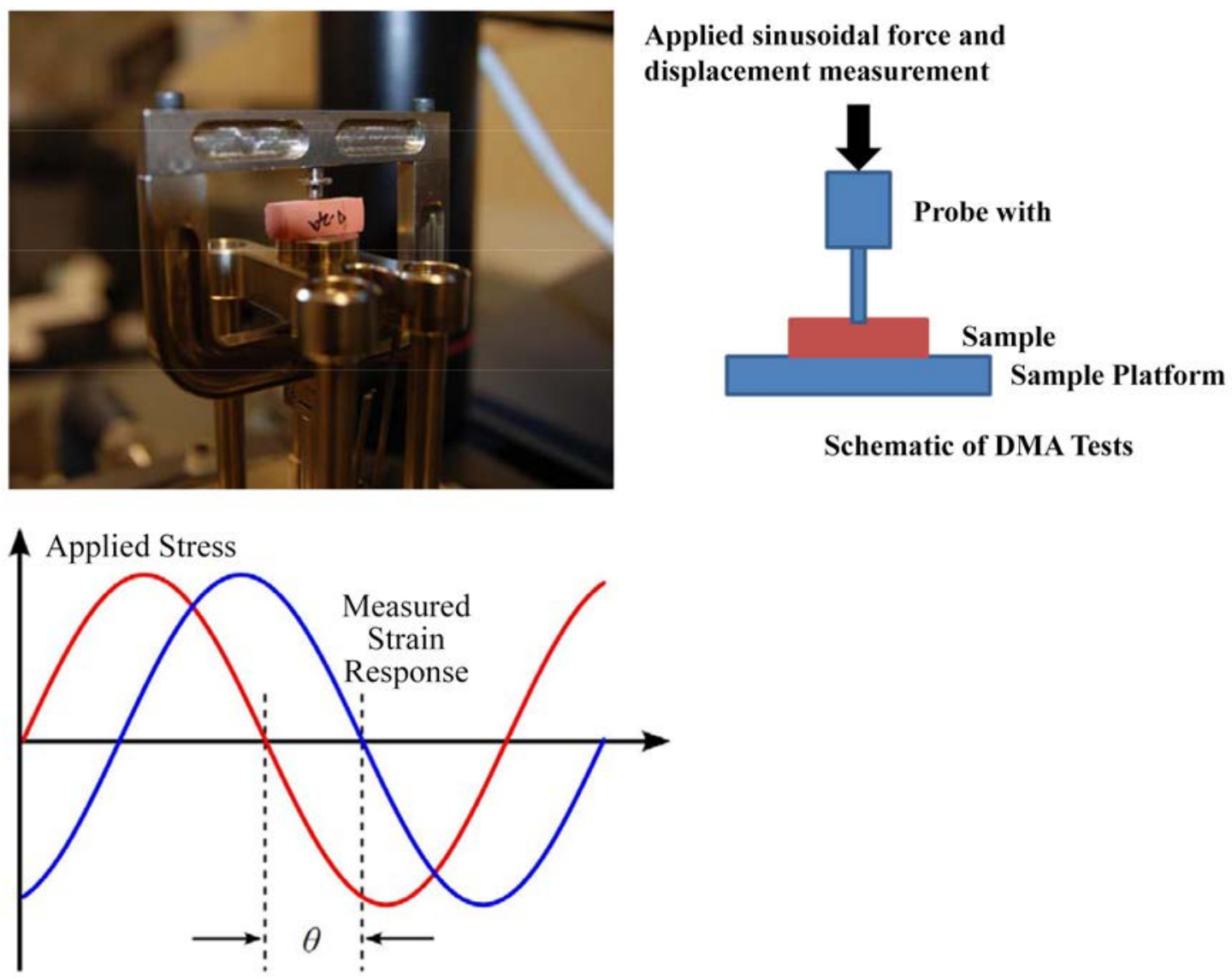

Figure 3.7. Illustration of DMA Instrument with Simple Schematic and Input/Output Waveforms for Analysis

Figure 3.8 illustrates how information from DMA measurements can be used to compare changes in materials based on process conditions or changes in the polymer structure and morphology. Given the types of information that are available from these measurements, it is apparent that DMA is an extremely valuable tool for polymer characterization. 


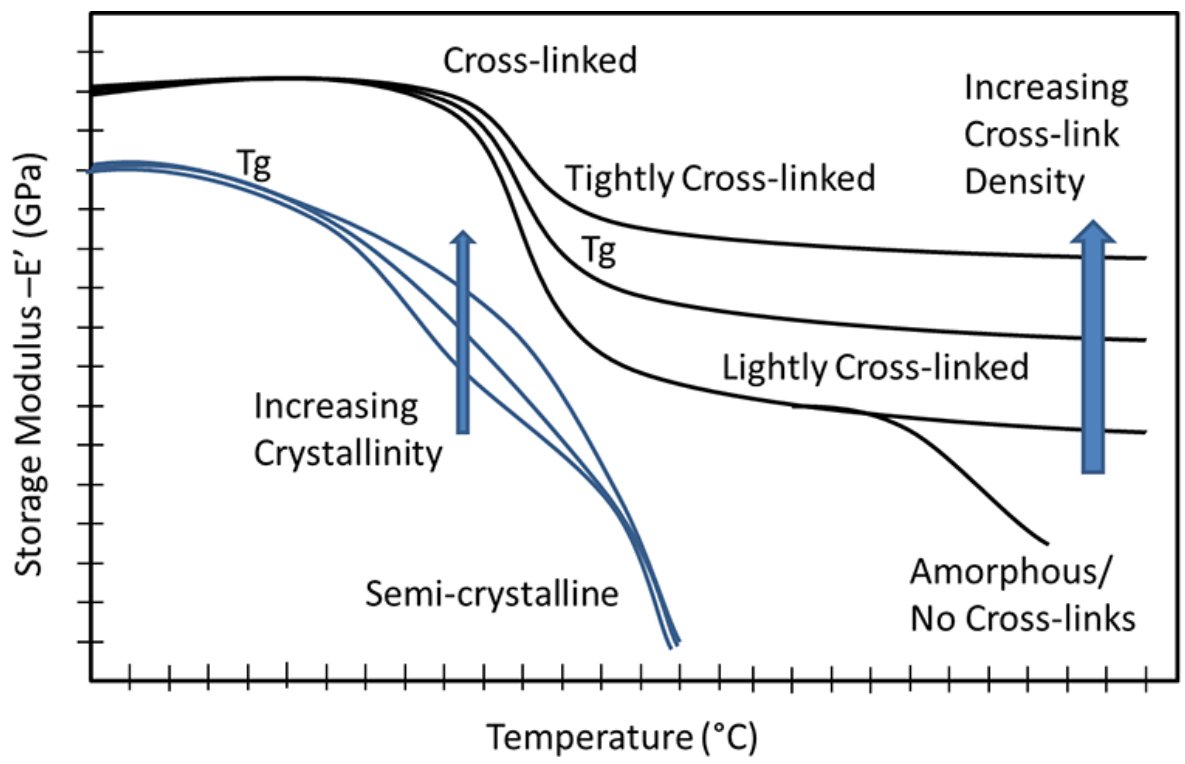

Figure 3.8. DMA Data Schematic Illustrating the Available Information Determined by DMA

DMA storage modulus data is related to tensile modulus for the material. This type of data is valuable for assessing techniques for acoustic measurements and the change in properties as it thermal degrades. In Figure 3.9, the DMA storage and loss modulus data from the Okoguard jumper cable is shown in the baseline, unaged condition. The data reveals a glass transition temperature of $-48^{\circ} \mathrm{C}$ and very compliant soft material at room temperature relative to its high stiffness below the glass transition. The rise in storage modulus of the Okoguard cable with oven aging is illustrated in Figure 3.10.

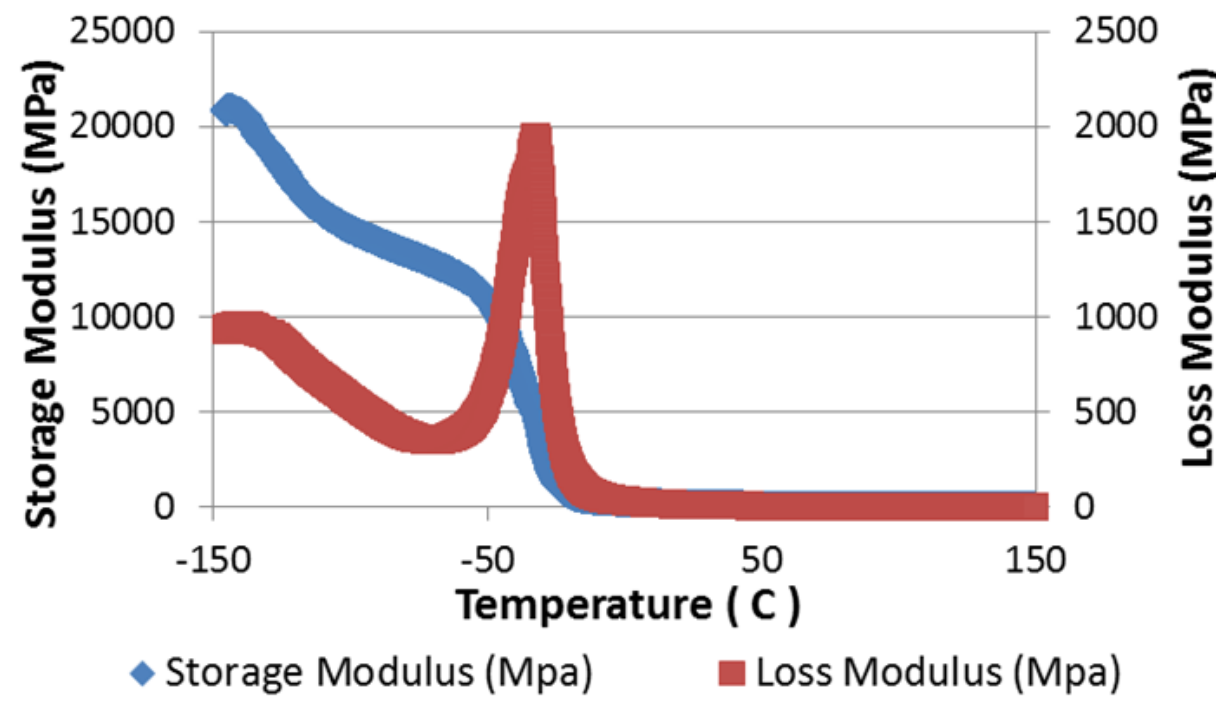

Figure 3.9. DMA Data for Unaged Okoguard Jumper Cable 


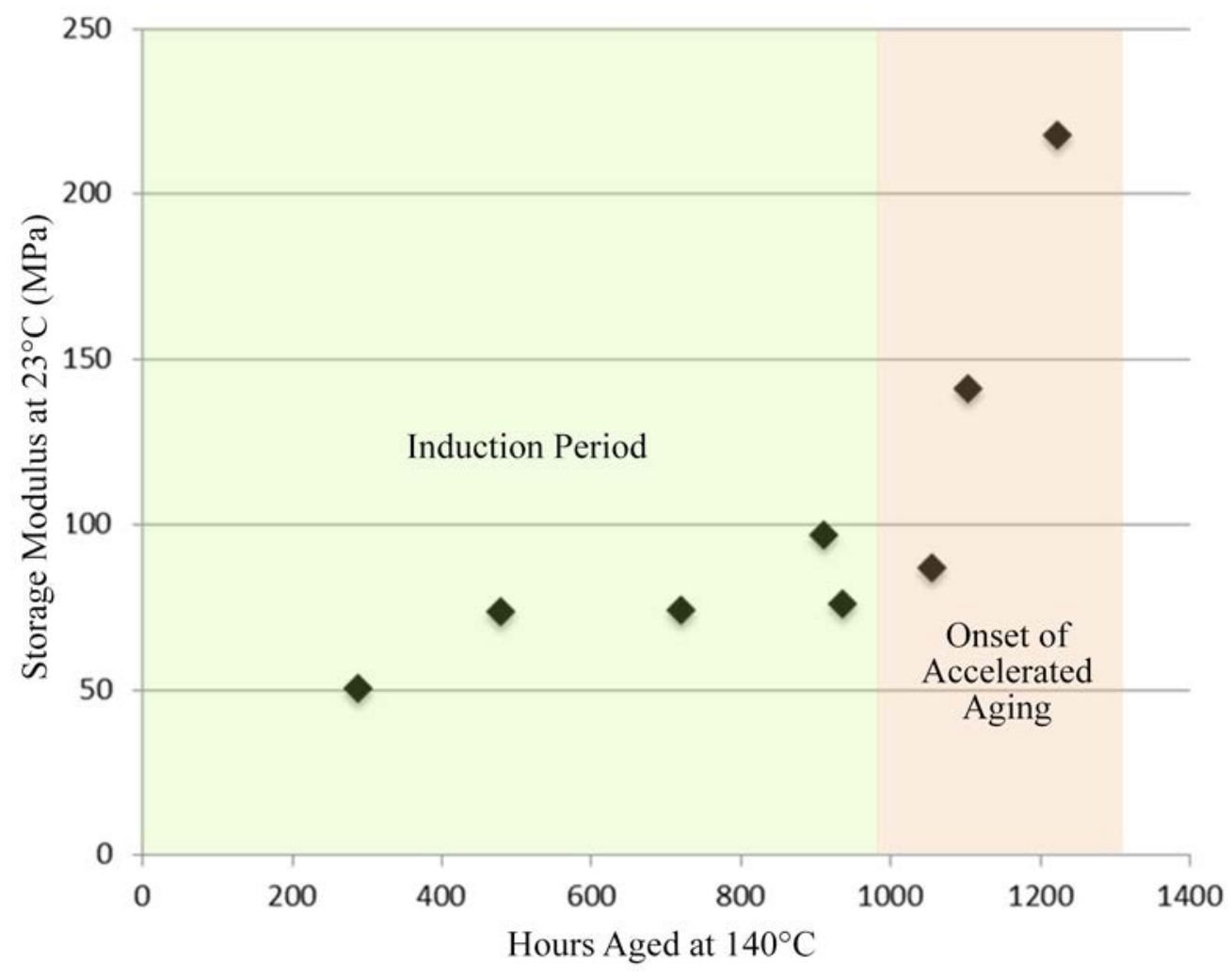

Figure 3.10. Storage Modulus of $140^{\circ} \mathrm{C}$ Thermally Aged EPR Okoguard Jumper Cable

\subsubsection{Ultrasonic Measurements of Sound Speed and Attenuation}

Acoustic wave interactions with solids depend on mechanical properties of the material (Pao 1983) such as density and elastic constants (Krautkrämer and Krautkrämer 1990). In solids, these waves are also referred to as elastic or stress waves. Variability in the spatial distribution of these properties results in an inhomogeneous material, with resulting spatial variability of the acoustic properties (velocity, acoustic impedance, etc.). In contrast, in an anisotropic material, the acoustic property is a function of direction of propagation.

The behavior of acoustic waves in solids is also a function of the wave mode. The three bulk wave modes usually considered are longitudinal or compressional (called L or P), horizontally polarized shear (SH), and vertically polarized shear (SV). In addition to these modes, surface and plate wave modes (and other modes) can also be generated, depending on the particular parameters and component geometry. In particular, specific modes may be generated that interact with the boundaries of a structure, and are able to be guided along the structure for long distances (10s of meters or more). Such guided waves are of interest when examining inaccessible portions of structures.

The wave speed $c_{l}$ in solids for compressional waves is given by (Krautkrämer and Krautkrämer 1990): 


$$
c_{l}=\sqrt{\frac{E}{\xi} \frac{1-\mu}{(1-\mu)(1-2 \mu)}}
$$

where $E$ is the modulus of elasticity (units: $\left.N / \mathrm{m}^{2}\right)$, $\xi$ is the material density $\left(\mathrm{kg} / \mathrm{m}^{3}\right)$, and $\mu$ is Poisson's ratio for the material (a dimensionless quantity). For plane waves or spherical waves, the sound pressure $p$ (related to the applied force) and (compressional) displacement $\zeta$ are related by (Krautkrämer and Krautkrämer 1990):

$$
p=\xi c_{l} \omega \zeta=Z \omega \zeta
$$

where $Z$ is the acoustic impedance.

These two relations indicate that sound speed measurements may be a proxy to measuring the elastic modulus. When the velocity measurements are coupled with measurements of density of the medium, the resulting acoustic impedance of the medium may, in turn, be capable of providing a quantity that is, in form, similar to the indenter modulus.

As a consequence, in this study, we investigate how acoustic velocity (correlated to elastic modulus) compares to the mechanical measurements made from the indenter method. Literature indicates that acoustic methods have been evaluated to some extent and that good correlations exist between velocity and breaking elongation (Ikehara et al. 1998). A challenge with velocity measurements in polymers is the generally higher level of damping introduced by these materials. Thus, initial trials have been focused on developing the measurement protocol for acoustic velocity measurement in EPR rubber.

While multiple protocols are possible for velocity measurement, we will be examining longitudinal and surface wave velocity measurement methods. In the longitudinal wave approach, sound is propagated through the thickness of the specimen. The applied acoustic energy is reflected from the surfaces of the specimen (shown in Figure 3.11) and received by the transmitting transducer (or a second receiving transducer if one is used). The transit time through the thickness of the specimen (corresponding to an acoustic path length of twice the thickness) is generally used to compute the sound velocity. A delay line (such as an acrylic block) may be used to increase the stand-off of the transducer from the specimen, and provide better separation of the responses from the near and far surfaces. 


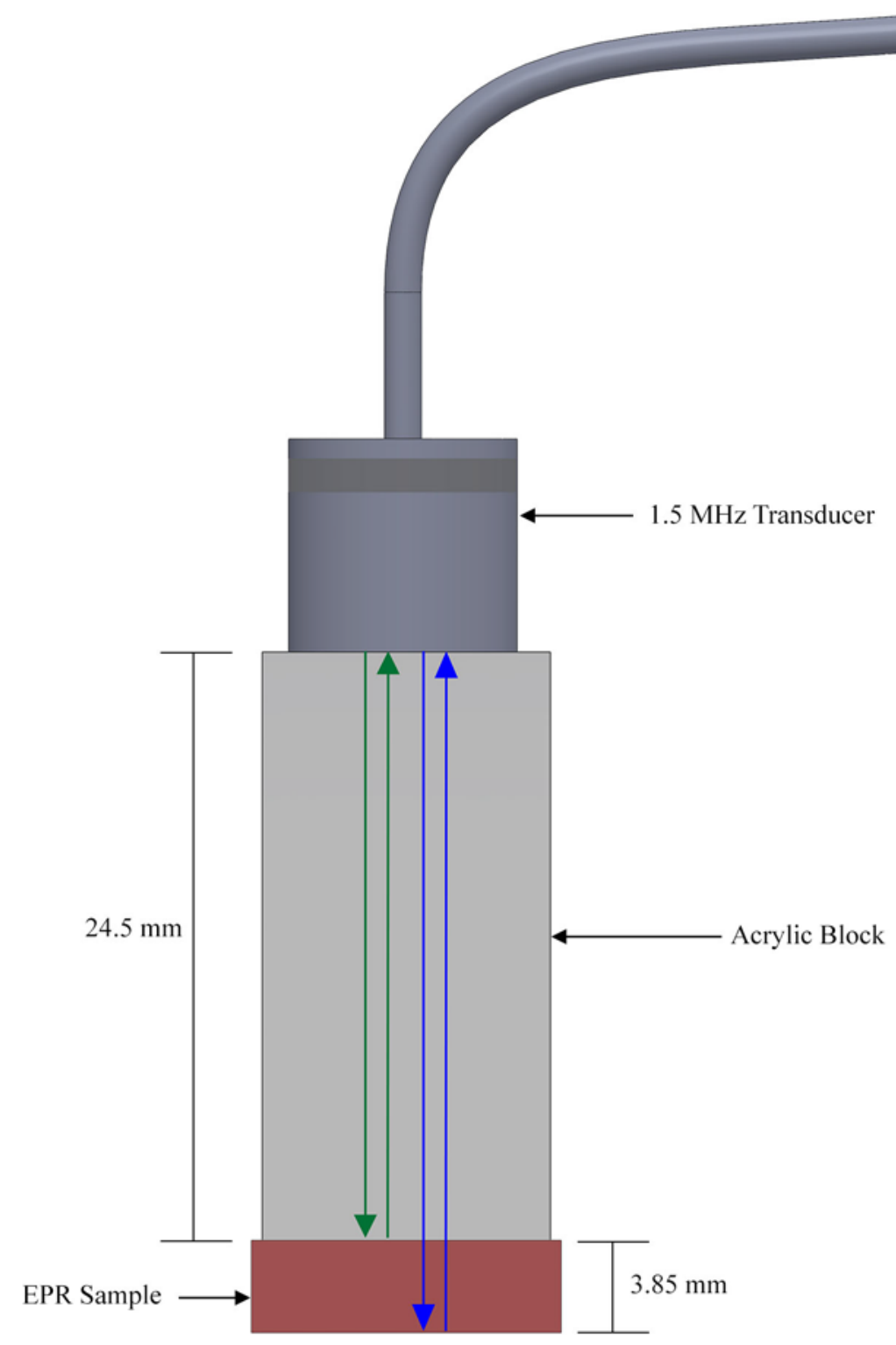

Figure 3.11. Ultrasonic Transducer Configuration for Standoff Measurement of EPR Sample

Surface waves differ from bulk longitudinal and shear wave modes in that the waves follow the contour of the surface and penetrate the medium to only about one wavelength deep from the surface (Roberts 1990; Ensminger and Bond 2011). While two types of surface waves are possible, Rayleigh surface waves are the more common form used in ultrasonic measurements. The velocities of Rayleigh surface waves $c_{R}$, bulk longitudinal waves $c_{l}$, and shear waves $c_{s}$ are related by Ensminger and Bond (2011):

$$
\frac{c_{R}^{6}}{c_{s}^{6}}-8 \frac{c_{R}^{4}}{c_{s}^{4}}+c_{R}^{2}\left(\frac{24}{c_{s}^{2}}-\frac{16}{c_{l}^{2}}\right)-16\left(1-\frac{c_{s}^{2}}{c_{l}^{2}}\right)=0
$$


Surface wave generation in solids uses the fact that, as sound propagates from one medium to another, it is refracted, with the propagation angle $\theta_{2}$ in the second medium a function of the incident angle $\theta_{1}$, and the ratio of the sound velocities in the two media:

$$
\frac{\sin \left(\theta_{1}\right)}{c_{1}}=\frac{\sin \left(\theta_{2}\right)}{c_{2}}
$$

Clearly, at a certain angle (called the second critical angle), the refracted energy propagates along the interface between the two media $\left(\theta_{2}=90^{\circ}\right)$. Measurements using a second spatially separated probe, also at the critical angle, can provide the necessary transit time used to compute the wave velocity.

The samples that are being aged and characterized by DMA will be acoustically examined using a low-frequency ultrasonic technique that provides a measurement value that can be directly compared to the DMA measurements. Initial trials used a section of unaged EPR that has been machined into a relatively uniform thickness. The goal of this initial trial was to establish the measurement protocol for bulk and surface wave velocity measurements. This small sample (and future aged samples) was examined using the PNNL "SNAP” ultrasonic system as well as the acoustic microscopy system shown in Figure 3.12.
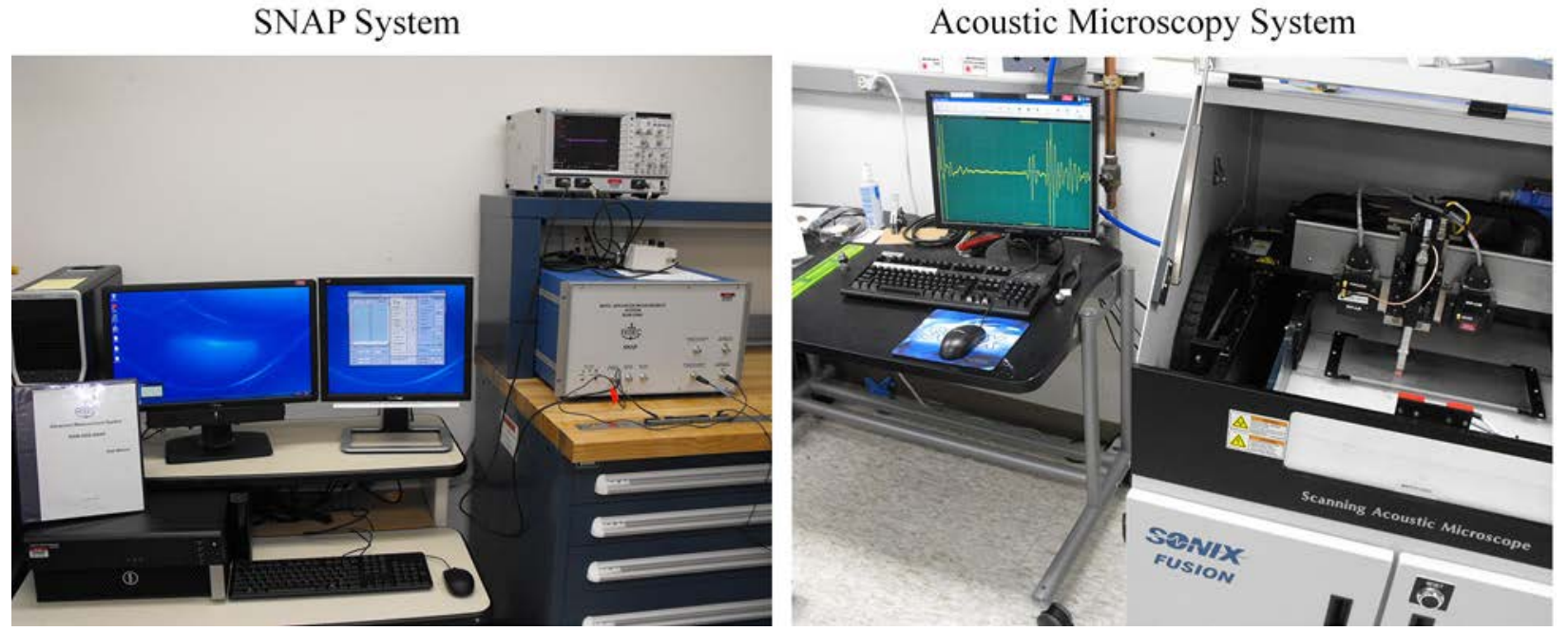

Figure 3.12. PNNL SNAP Ultrasonic System and PNNL Acoustic Microscopy System

The SNAP system provides the ability to obtain linear and nonlinear ultrasonic measurements, and includes the ability to perform sound speed and attenuation measurements. Both longitudinal and surface wave measurement protocols are supported. The acoustic microscope extends the measurement capability to higher frequencies (in excess of $20 \mathrm{MHz}$ ) and enables high-precision mapping of acoustic property spatial variation in small specimens. Instrument calibration in both cases uses materials with known acoustic properties (such as acrylic or glass). 


\subsection{Measurement Techniques for Electrical Properties}

Prior to investigating different electrical nondestructive methods as possible measurement techniques for cable degradation, a consensus understanding of what research has already been accomplished was necessary. The International Atomic Energy Agency (IAEA) Nuclear Energy Series Assessing and Managing Cable Ageing in Nuclear Power Plants (IAEA 2012) and a recent workshop Light Water Reactor Sustainability (LWRS) Program - Non-Destructive Evaluation (NDE) R\&D Roadmap for Determining Remaining Useful Life of Aging Cables in Nuclear Power Plants (Simmons et al. 2012) proved to be valuable resources in identifying the latest research applicable to electrical measurements on cables. From these reports, Table 3.1 identifies electrical measurements and their advantages and disadvantages. The main advantage to electrical measurements is the possibility of evaluating the entire cable length in-situ. The main disadvantage is that they are not very sensitive to insulation degradation.

Table 3.1. Commercially Available Techniques for Cable Inspection

\begin{tabular}{|c|c|c|}
\hline Inspection Method & Advantages & Disadvantages \\
\hline $\begin{array}{l}\text { Time-Frequency Domain } \\
\text { Reflectometry (TDR and FDR) }\end{array}$ & $\begin{array}{l}\text { Commonly used for determining the } \\
\text { condition of instrumentation, } \\
\text { control, and power cables where } \\
\text { they are inaccessible. }\end{array}$ & $\begin{array}{l}\text { Currently intrusive, requires } \\
\text { disconnecting the cables to install } \\
\text { instrumentation. }\end{array}$ \\
\hline Insulation Resistance & $\begin{array}{l}\text { Commonly performed in industry to } \\
\text { determine the condition of the cable } \\
\text { insulation. }\end{array}$ & $\begin{array}{l}\text { Currently intrusive, requires } \\
\text { disconnecting the cables to install } \\
\text { instrumentation. }\end{array}$ \\
\hline $\begin{array}{l}\text { Inductance/Capacitance/ } \\
\text { Resistance (LCR) }\end{array}$ & $\begin{array}{l}\text { Good for detecting changes in } \\
\text { electrical circuit (cable and } \\
\text { termination) by trending changes in } \\
\text { inductance, capacitance, and } \\
\text { resistance. }\end{array}$ & $\begin{array}{l}\text { Currently intrusive, requires } \\
\text { disconnecting cable at one end. } \\
\text { Does not indicate location or } \\
\text { cause of change in measurement. }\end{array}$ \\
\hline Tan Delta $(\operatorname{Tan} \delta)$ & $\begin{array}{l}\text { Determines changes in insulation } \\
\text { (dielectric) properties by measuring } \\
\text { change in dielectric loss angle. Can } \\
\text { measure aging effects over entire } \\
\text { cable length. }\end{array}$ & $\begin{array}{l}\text { Intrusive, requires decoupling } \\
\text { both ends. Single number from } \\
\text { long cable makes isolating } \\
\text { location of aging section difficult. } \\
\text { Loss angle may be trended; } \\
\text { however, single measurement } \\
\text { insufficient to estimate remaining } \\
\text { life. }\end{array}$ \\
\hline Partial Discharge & $\begin{array}{l}\text { Good for determining voids or } \\
\text { defects in insulators of medium } \\
\text { voltage cables. }\end{array}$ & $\begin{array}{l}\text { Test can damage the insulator } \\
\text { with localized heating that causes } \\
\text { degradation. }\end{array}$ \\
\hline \multicolumn{3}{|c|}{$\begin{array}{l}\text { FDR }=\text { frequency domain reflectometry } \\
\mathrm{LCR}=\text { inductance/capacitance/resistance } \\
\mathrm{TDR}=\text { time domain reflectometry }\end{array}$} \\
\hline
\end{tabular}

Prior to pursuing a nondestructive evaluation (NDE) technique for each of these inspection methods, a basic understanding of the underlying physics is needed. Therefore, it was necessary to establish criteria for further investigation. The criterion uses the requirement that the method to be evaluated must have the ability to perform a nondestructive test to determine electrical properties of the cable jackets and 
insulation without significant disturbance of the cables and connectors as they lay in-situ. Each of the electrical methods described in the table was considered intrusive in that one or both ends of the cable would have to be disconnected. Therefore, the circuit that is operated by that cable would go off-line.

The initial premise is then to address electrical measurement techniques that may potentially be amenable to in-situ (online) assessment of cable insulation degradation. With the exception of reflectometry (ideal for identifying faults in the conductors in cables) and partial discharge (which may, under certain conditions, result in added degradation to the cable insulation), the remaining methods each can potentially be used in-situ. Initial evaluations resulted in investigating the Tan $\delta$ method as a first step towards understanding the physics underpinning these types of measurements. Other methods (insulation resistance and LCR) will be examined in future phases.

\subsubsection{Tan $\delta$ Measurement Technique}

The tan delta technique or Tan $\delta$ can be derived from one of Maxwell's four equations, which relates the magnetic field intensity to the electric field intensity. Expressed in phasor form, the equation contains the relationship between the conduction current density $(\sigma \mathcal{E})$ and the displacement current density $\left(j \omega \varepsilon^{\prime}\right)$ for dielectric materials.

$$
\nabla \times \mathfrak{H}=\sigma \mathcal{E}+j \omega \varepsilon^{\prime} \mathcal{E}\left(\mathrm{Am}^{-2}\right)
$$

where: $\quad \mathscr{H}=$ magnetic field intensity $(\mathrm{A} / \mathrm{m})$

$\mathcal{E}=$ electric field intensity $(\mathrm{V} / \mathrm{m})$

$\sigma=$ conductivity $(\mho / \mathrm{m})$

$\omega=$ angular frequency $(\mathrm{rad} / \mathrm{sec})$

$\varepsilon^{\prime}=$ real portion of the complex permittivity where $\varepsilon=\varepsilon^{\prime}-j \varepsilon^{\prime \prime}(\mathrm{F} / \mathrm{m})$

The relative permittivity $\varepsilon_{r}$ describes how a specific material will interact with an applied electric field. Known as the dielectric constant of the material, it is derived from the permittivity of free space $\varepsilon_{0}=8.854 \times 10^{-12} \mathrm{~F} / \mathrm{m}$ where $\varepsilon=\varepsilon_{r} \varepsilon_{0}$.

This complex permittivity can therefore be written as $\varepsilon_{r}=\varepsilon_{r}^{\prime}-j \varepsilon_{r}^{\prime \prime}$ and shown on a simple vector diagram (Figure 3.13).

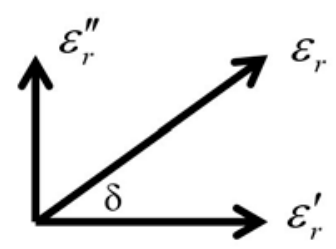

Figure 3.13. Phasor Relationship between the Real and Imaginary Components of Permittivity 
The tangent of the angle $\delta$ between them is the ratio of these two vector quantities and is a measure of the ratio of energy from the applied electric field that is stored in a specific material to the amount dissipated or lost. This quantity is known as the loss tangent and defined as:

$$
\operatorname{Tan} \delta=\frac{\varepsilon_{r}^{\prime \prime}}{\varepsilon_{r}^{\prime}}
$$

for that material.

To understand the fundamental relationship between aged samples of electrical cables, PNNL employed a vector network analyzer (VNA) to acquire complex permittivity measurements. The VNA used was an Agilent Technologies model E8361A with integrated software and an associated hightemperature coaxial dielectric probe with a frequency bandwidth of $200 \mathrm{MHz}$ to $20 \mathrm{GHz}$ as shown in Figure 3.14.

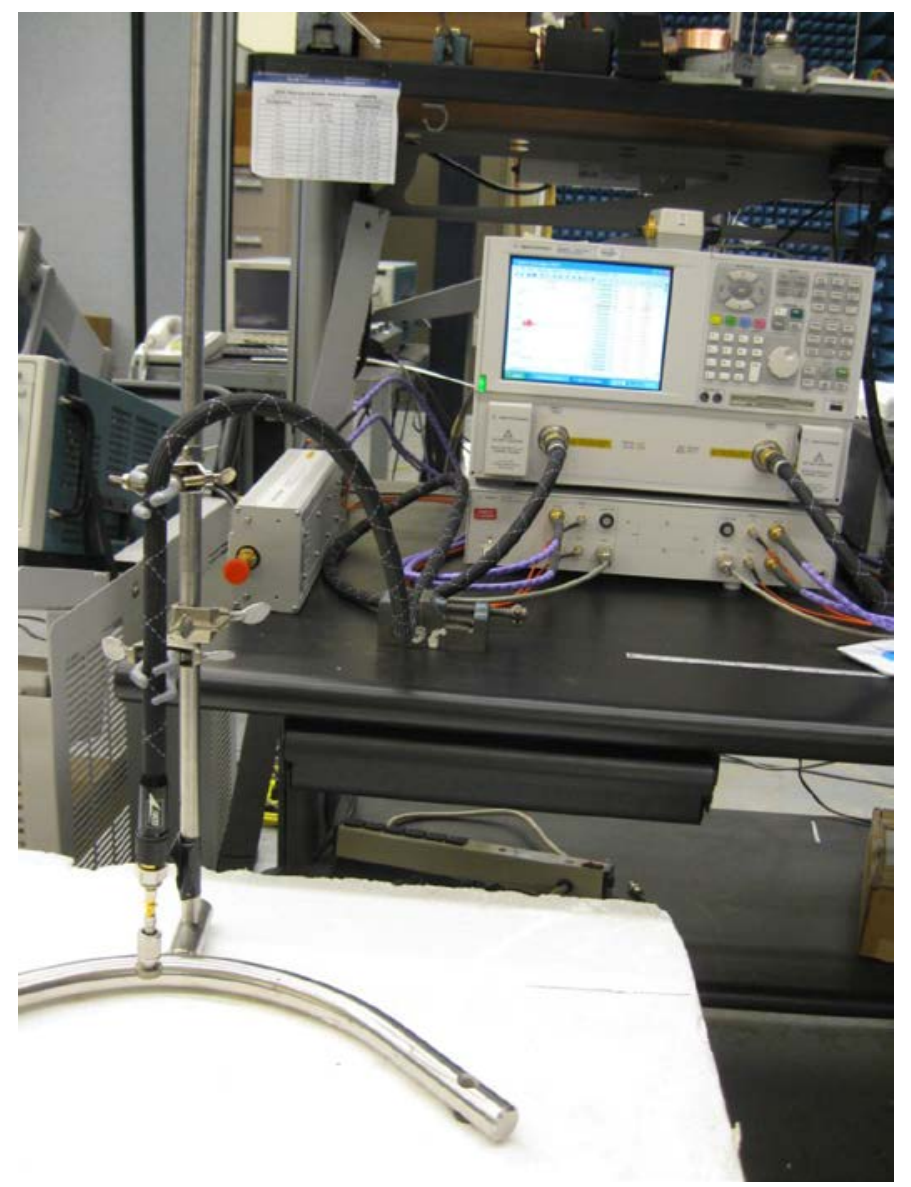

Figure 3.14. Agilent Technologies Vector Network Analyzer

Calibration of the VNA is essential to assure accurate, repeatable measurements of the complex permittivity. Calibration of the VNA is performed by subjecting the measuring probe to air, then shorting 
the probe using a short-circuit standard provided by Agilent, and then placing the probe in deionized water at a reference temperature. The reference temperature was $23^{\circ} \mathrm{C}$ for these measurements. Figure 3.15 shows each of these steps in the calibration process.
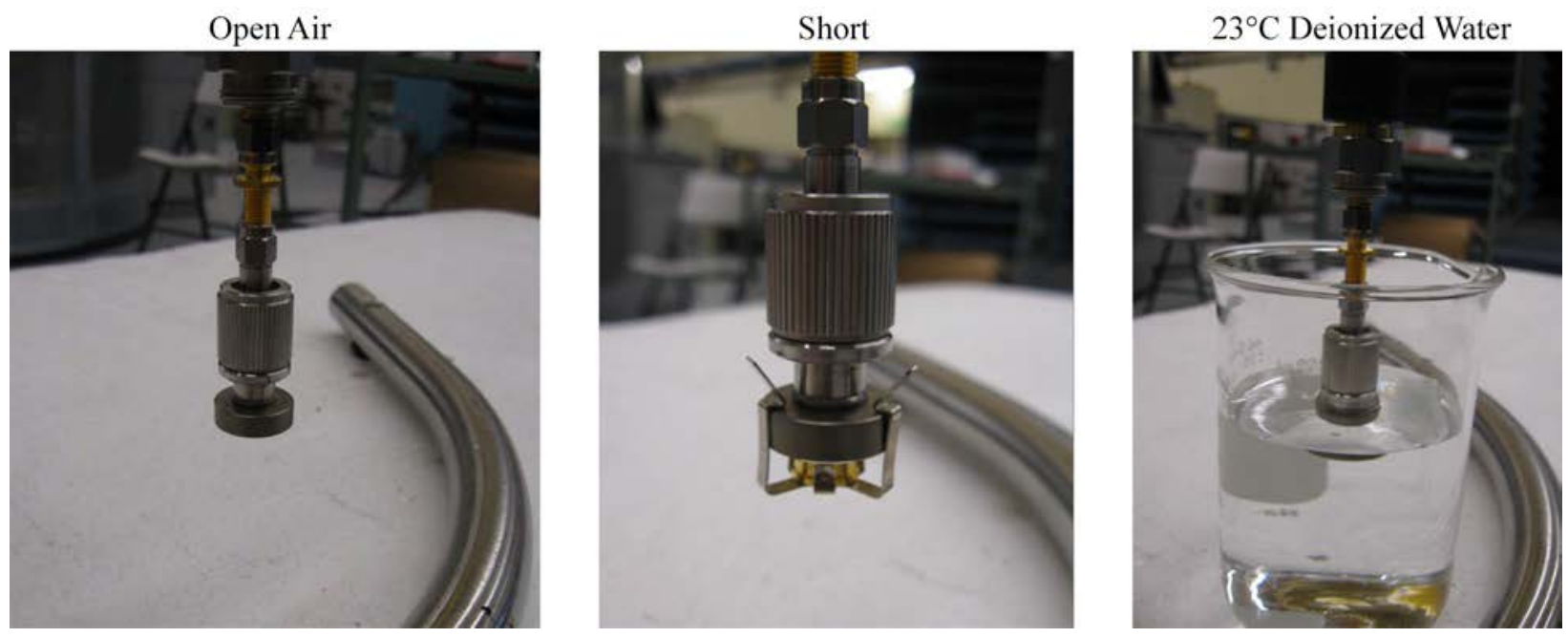

Figure 3.15. Steps of Calibration Process

Once calibration was completed, measurements of the complex permittivity of known materials were used to verify the accuracy of the calibration. Plots shown in Figure 3.16 show the values attained for air and Rexolite.

- $\operatorname{Air}\left(\varepsilon^{\prime}=1.0, \quad \varepsilon^{\prime \prime}=0.0\right)$

- Rexolite $\left(\varepsilon^{\prime}=2.5, \quad \varepsilon^{\prime \prime}=\sim 0.0\right)$

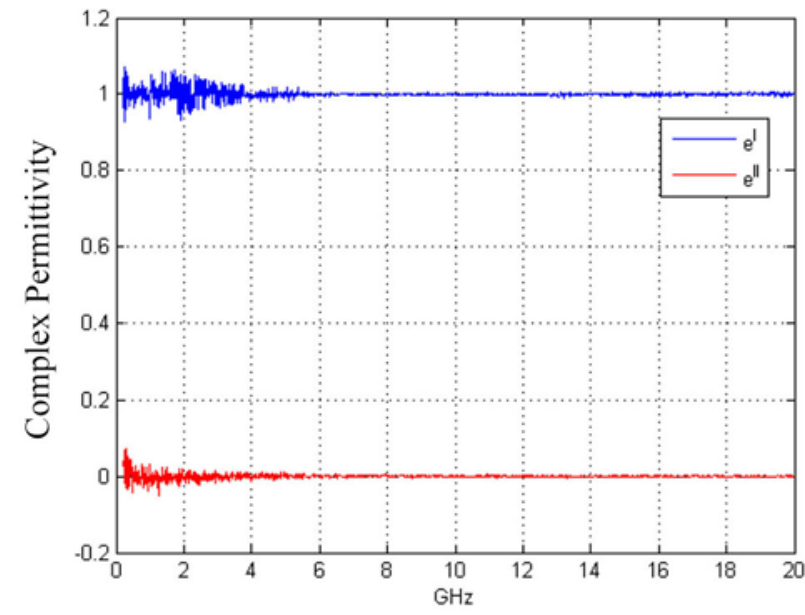

Complex Permittivity Measurements of Air

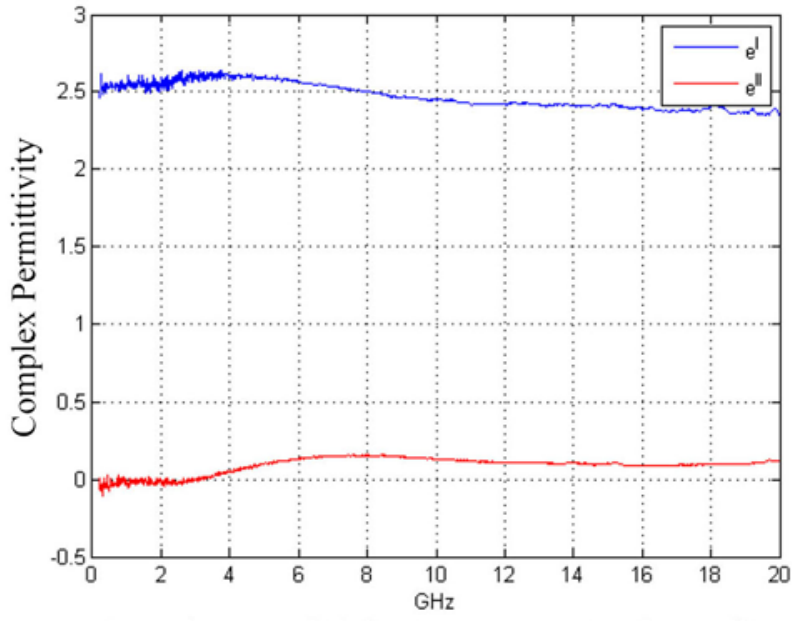

Complex Permittivity Measurements of Rexolite

Figure 3.16. Complex Permittivity Measurements Obtained during Calibration Process 


\subsection{Summary of Data}

The measurement techniques described above were used to acquire data on samples of unaged and aged EPR rubber, described in Section 3.1. As the initial focus of the acoustic measurements was on establishing a measurement protocol, only unaged specimens were used at this stage. On the other hand, the electrical measurement protocol is relatively mature, and was used to perform measurements and initial analysis on a set of unaged and aged specimens. The protocols (for acoustic measurements) and the data (for both electrical and acoustic measurements) are summarized in this section.

\subsection{Acoustic Measurements}

Preliminary measurements were performed using the acoustic microscopy system on a new unaged sample of cable (Okoguard) that was machined into a uniform thickness. Figure 4.1 shows the sample in the acoustic microscope.

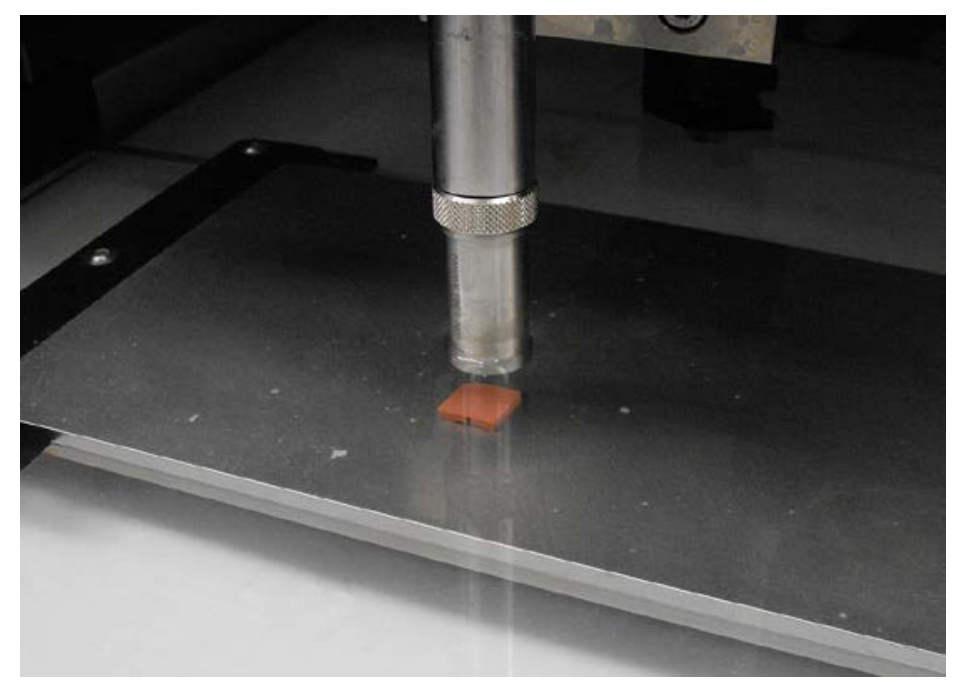

Figure 4.1. EPR Sample - Immersion Technique

The measured dimensions of the sample were $12.9-\mathrm{mm} \times 12.4-\mathrm{mm} \times 3.85$-mm thick (measured with Vernier calipers). The objective of this first preliminary testing was to ascertain whether a sound wave could be transmitted through the material with minimal attenuation so that a reasonable back-wall reflection could be imaged and measured. The initial transducer used was an unfocused 1-MHz immersion transducer, which was the lowest frequency transducer available that was applicable to this type of measurement. Shown in Figure 4.2 is an A-scan display of the time of flight between the front surface of the cable section and the back surface of the cable section using the acoustic microscope. The large back-surface reflection stems from the plate the sample is sitting on. If the sample were suspended, then the back-wall reflection would be smaller than the front-wall reflector. The method was sufficient to indicate that an adequate signal-to-noise ratio was available to make accurate measurements of transit time. This transit time of flight was measured as $5.084 \mu \mathrm{sec}$. Using the mechanically measured value of $3.85-\mathrm{mm}$ for the thickness provides a velocity measurement of $1.51 \mathrm{~mm} / \mu \mathrm{sec}$. 


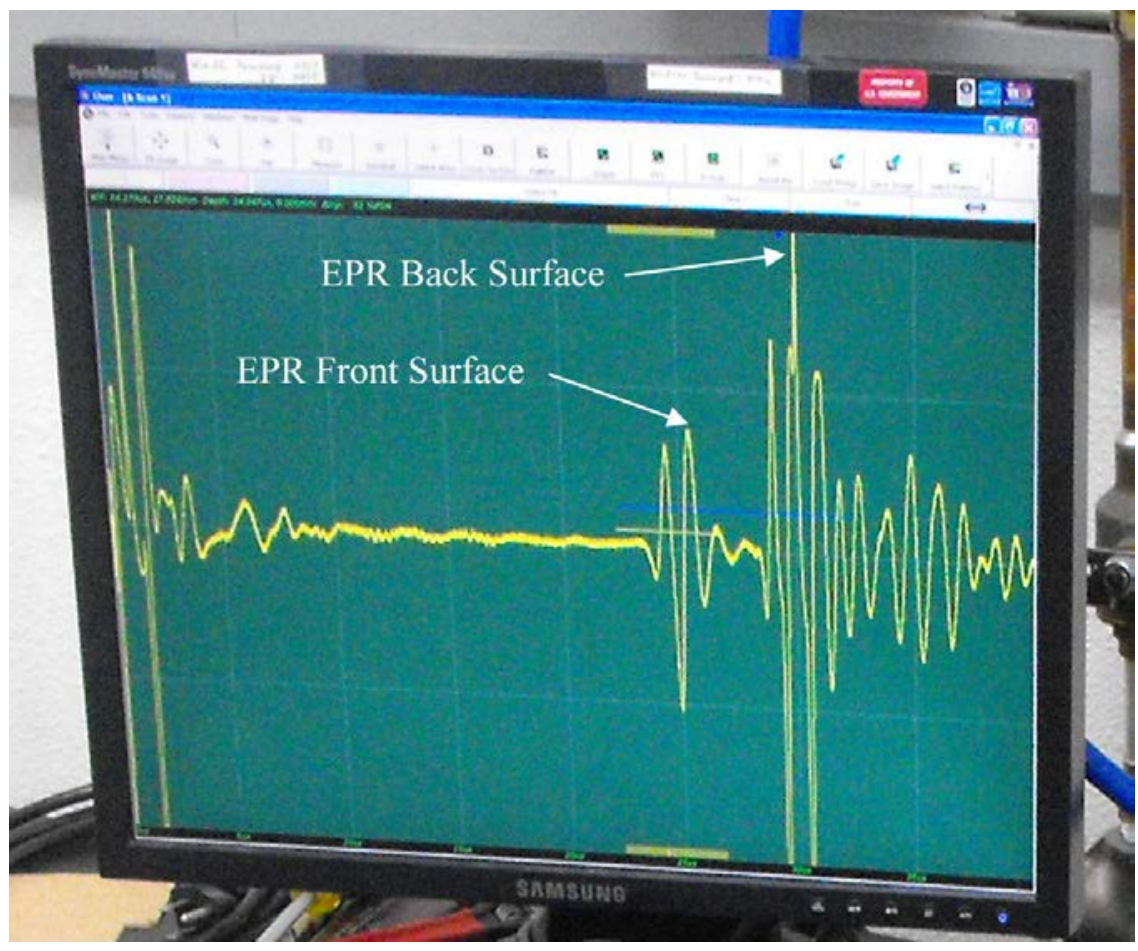

Figure 4.2. A-Scan Display from Front and Back Wall of Cable Sample

Additional preliminary measurements were made using contact techniques that used longitudinal waves and surface waves. The longitudinal wave technique used a 1.5-MHz, 3/8-inch diameter transducer and an acrylic block to provide a standoff distance. This provided a means to identify the front surface echo from the cable outer diameter and the back surface echo from the cable inner diameter. Figure 4.3 shows the signal at the first back wall of acrylic block (through acrylic block to front face of the EPR sample) and then through the EPR to the first back wall of the EPR sample. Transit time was measured at $5.156 \mu \mathrm{sec}$, which results in a velocity calculation in the EPR at $1.49 \mathrm{~mm} / \mu \mathrm{sec}$, which correlates well with the immersion test. Measurement precision depends on a number of quantities including error in the mechanical thickness measurement for the specimens and error in identifying constant-phase points on the A-scan, and will be quantified as additional measurements are made with aged and unaged specimens.

To identify whether a surface wave could be transmitted, a contact transducer with a high-angle wedge (greater than approximately $70^{\circ}$ from normal to generate a surface wave) was used. Figure 4.4 shows the arrangement and the screen display of the surface wave signal. Although not evident in the photograph, there is an approximately 1-mm separation between the transmit and receive wedges. Bringing the two wedges in contact results in an additional signal (transmitted directly through the two wedges) that arrives prior to the desired surface wave signal. Further investigation will be needed to ascertain whether this technique can provide a reliable method for measuring acoustic velocity. 


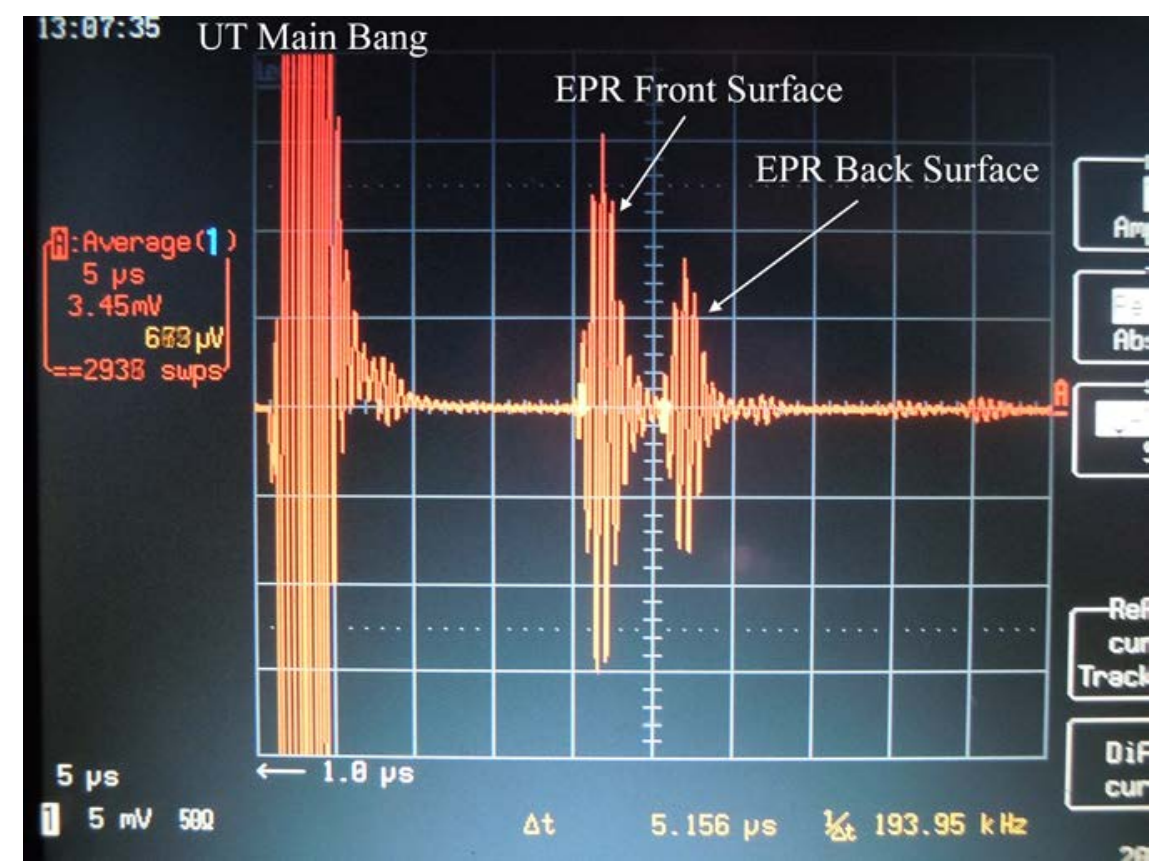

Figure 4.3. UT Signals from the Front and Back Face of the EPR Sample

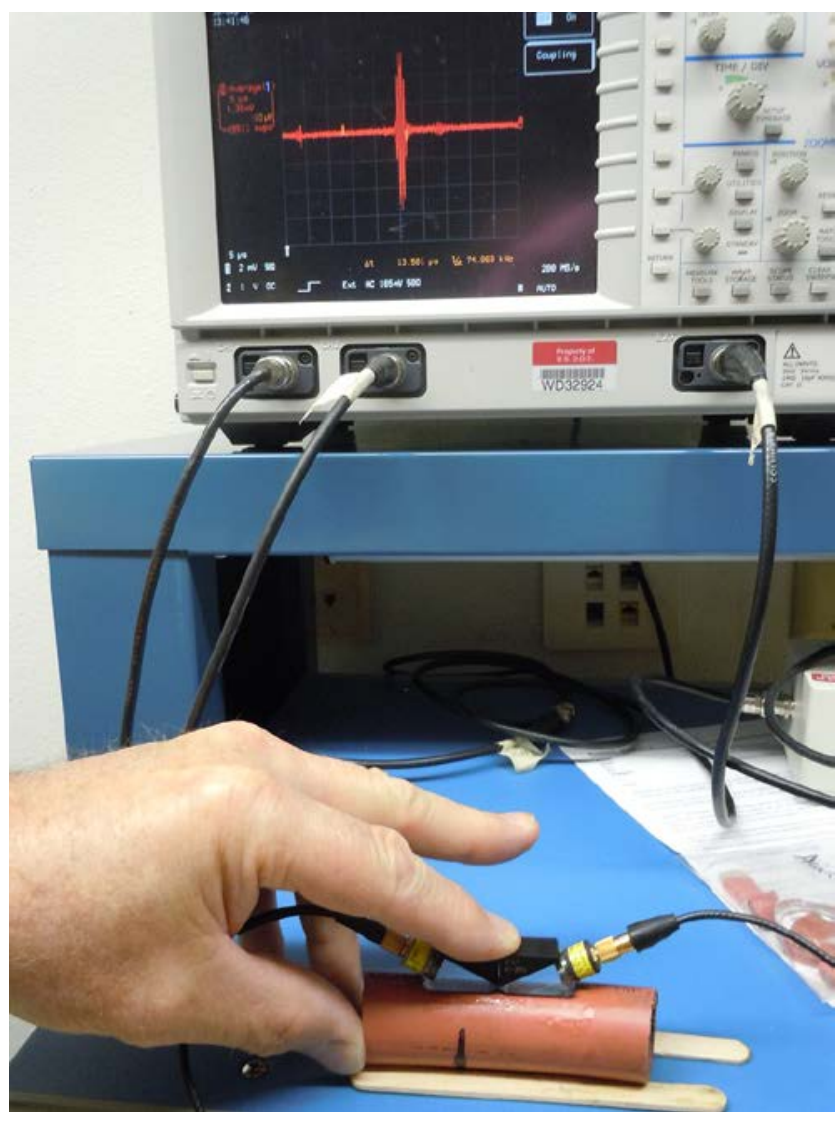

Figure 4.4. Surface Wave Technique 


\subsection{Electrical Measurements}

A total of 24 cable insulation samples were generated that represent 12 different cable degradation levels, with 2 samples per degradation level. A list of the cable insulation samples is shown below in Table 4.1.

Table 4.1. Cable Insulation Sample Sets

\begin{tabular}{ccc}
\hline Sample Name & Quantity & \multicolumn{1}{c}{ Description } \\
\hline As Received & 2 & Cable insulation as received \\
$288 \mathrm{hrs}$ & 2 & Cable insulation exposed to $140^{\circ} \mathrm{C}$ for $288 \mathrm{hrs}$ \\
$480 \mathrm{hrs}$ & 2 & Cable insulation exposed to $140^{\circ} \mathrm{C}$ for $480 \mathrm{hrs}$ \\
$720 \mathrm{hrs}$ & 2 & Cable insulation exposed to $140^{\circ} \mathrm{C}$ for $720 \mathrm{hrs}$ \\
$912 \mathrm{hrs}$ & 2 & Cable insulation exposed to $140^{\circ} \mathrm{C}$ for $912 \mathrm{hrs}$ \\
$936 \mathrm{hrs}$ & 2 & Cable insulation exposed to $140^{\circ} \mathrm{C}$ for $936 \mathrm{hrs}$ \\
$1080 \mathrm{hrs}$ & 2 & Cable insulation exposed to $140^{\circ} \mathrm{C}$ for $1080 \mathrm{hrs}$ \\
$1104 \mathrm{hrs}$ & 2 & Cable insulation exposed to $140^{\circ} \mathrm{C}$ for $1104 \mathrm{hrs}$ \\
$1128 \mathrm{hrs}$ & 2 & Cable insulation exposed to $140^{\circ} \mathrm{C}$ for $1128 \mathrm{hrs}$ \\
$1152 \mathrm{hrs}$ & 2 & Cable insulation exposed to $140^{\circ} \mathrm{C}$ for $1152 \mathrm{hrs}$ \\
$1200 \mathrm{hrs}$ & 2 & Cable insulation exposed to $140^{\circ} \mathrm{C}$ for $1200 \mathrm{hrs}$ \\
$1224 \mathrm{hrs}$ & 2 & Cable insulation exposed to $140^{\circ} \mathrm{C}$ for $1224 \mathrm{hrs}$ \\
\hline
\end{tabular}

The complex permittivity of the cable insulation samples was measured over a large bandwidth using the high-temperature dielectric probe shown in Section 3.4.1 to investigate if changes appeared in the real or imaginary component of the dielectric constant (permittivity) with respect to an increase in cable insulation age at $140^{\circ} \mathrm{C}$.

The requirements for accurate complex permittivity measurements include strong surface contact between the probe and the material under test. The presence of air gaps between the probe and material surface will significantly affect the material measurements. Because the dielectric probe measurement method assumes that the material is isotropic and homogeneous, the sample must appear infinitely wide and thick to the probe's fringing fields. For the probe used in this study, the diameter of the material under test must be no smaller than $20 \mathrm{~mm}$ in order to perform the measurement over a range of $200 \mathrm{MHz}$ to $20 \mathrm{GHz}$. The equation to satisfy the appearance of infinite electrical thickness is

$$
S=\frac{20}{\sqrt{\varepsilon^{\prime}}}
$$

where $S$ is the material thickness and $\varepsilon^{\prime}$ is the real part of the dielectric constant. For the material studied here, a nominal sample thickness of $12 \mathrm{~mm}$ is required. The thickness of the cable insulation samples was measured to be $5.8 \mathrm{~mm}$; therefore, the two samples for each cable age were measured together in a stacked configuration in order to obtain a thickness appropriate for the probe. A large amount of pressure was required to ensure that no air gap was present at the interface between the two samples and to flatten the curved cable insulation front surface. 
To ensure repeatability of the complex permittivity measurements, four data sets were collected per cable degradation level. For each of the four data sets collected, the position of the samples was swapped, resulting in measurements 1 and 3 having the same sample configuration, and measurements 2 and 4 having the same sample configuration. This tested the repeatability of data for each specimen set and also per each sample in that set.

For these measurements, the real component of the complex permittivity proved to be more responsive than the imaginary component to cable aging. The loss tangent measurements show a diminished response to increased aging because of the relatively small changes in the imaginary term when compared to the changes in the real term. Therefore, the results shown below focus on the real component versus frequency as a function of cable aging.

Figure 4.5 shows the measured real component of the complex permittivity for the cable insulator sample that was unaged. The data exhibits a somewhat noisy response from the $200 \mathrm{MHz}-5 \mathrm{GHz}$ band and an almost linear response from 6-9 GHz. From 10-20 GHz there is an inconstant modulation that occurs from sample to sample. This could be due to small but measurable differences in the data collection process such as non-uniformity of the probe position on the sample, the pressure applied during the measurement, or surface variations that result from the cable specimen bending when pressure is applied.

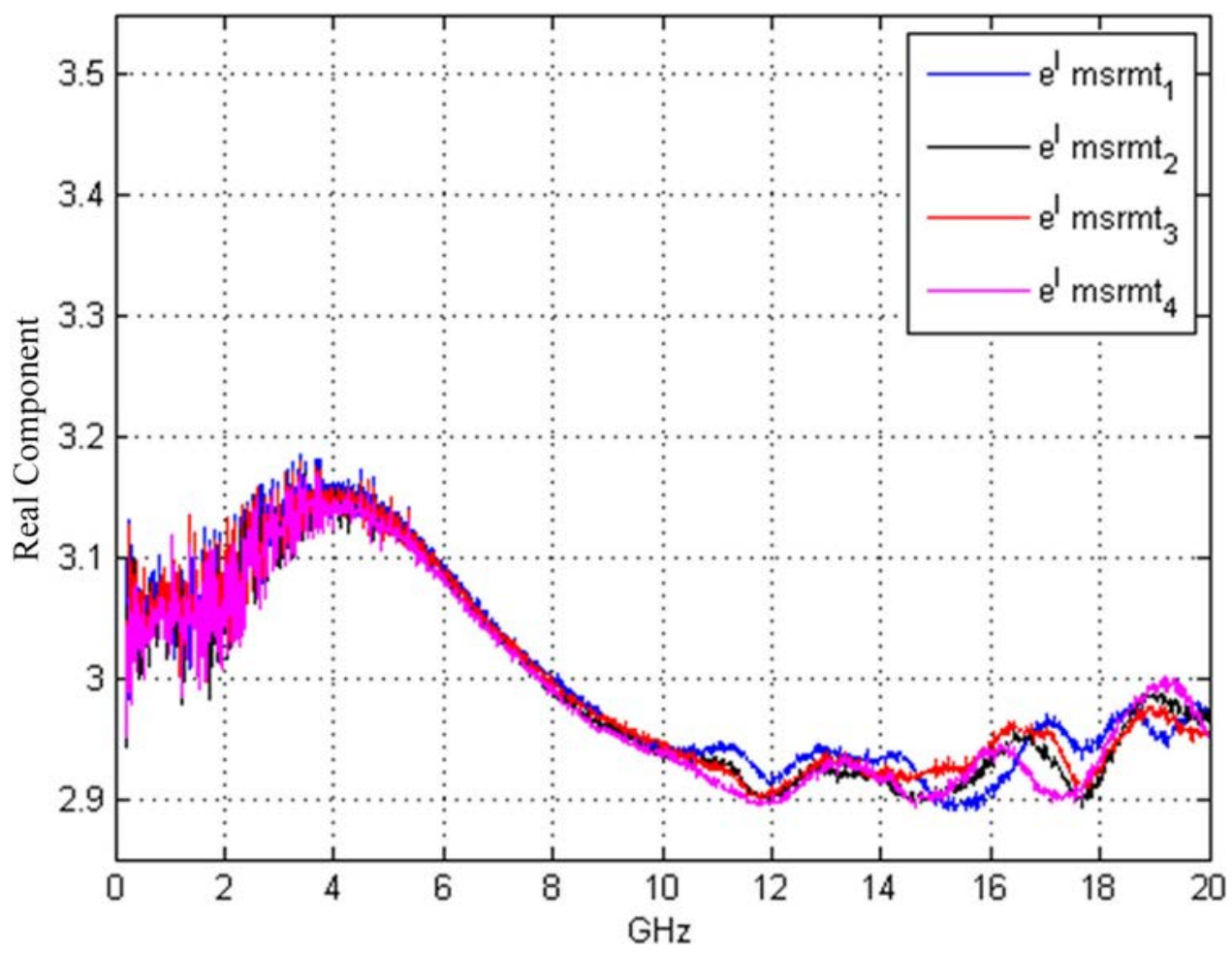

Figure 4.5. Initial (No Exposure) Dataset, Real Component of the Complex Permittivity

These measurements show a very consistent real dielectric constant for all four data sets, which provides confidence in the repeatability of the sample set. 
Samples that were exposed to $140^{\circ} \mathrm{C}$ for longer durations exhibited visible degradation of the exterior of the cable insulation. The sample portions that contained these visible marks created a variation in the real part of the complex permittivity between the two samples for that cable degradation level. However, as one can observe in Figure 4.6, measurements 1 and 3 correlate very well, whereas measurements 2 and 4 correlate well. For these measurements, the samples are in the same configuration for measurements 1 and 3, and they are in the same configuration for measurements 2 and 4; thus, the measurements are repeatable for each sample, but vary between the two sets because of the degradation on the cable insulation. Note that the aged samples exhibit a higher real part of the complex permittivity.

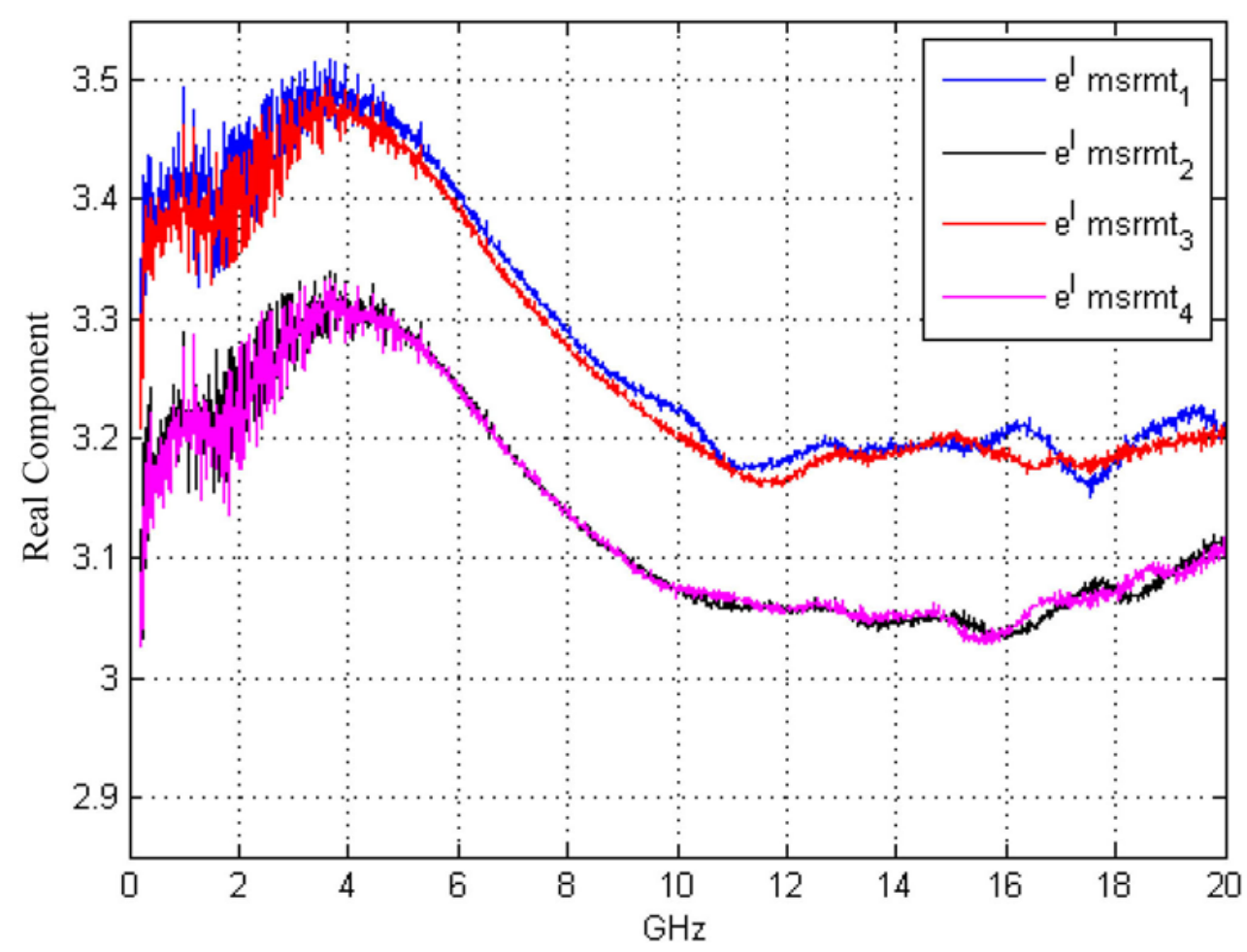

Figure 4.6. Sample Exposed to $140^{\circ} \mathrm{C}$ for 1200 Hours, Real Component of the Complex Permittivity

The complete set of data for all specimens is shown in Appendix A.

\subsubsection{Trend Analysis}

For every cable degradation level, reliable and consistent data was measured in the 6-9 GHz band. The data from 6-9 GHz was used to compare the measured real part of the dielectric constant for each degradation level. It was found that some samples had a greater amount of surface degradation and were measured to have a higher dielectric constant than samples at the same age that did not have the surface damage. Therefore, this was believed to not be an entirely accurate portrayal of the dielectric constant at that level of degradation. For completeness, two summary sets of plots are shown. The first plot displays measured data of the minimum trace values for the real part of the dielectric constant; this is believed to be the most accurate as it does not include the outliers that experienced some level of charring. The 
second set of data displays the measured data of the maximum trace values for the real part of the dielectric constant. This data set displays the wide variations in the dielectric constant for data corresponding to longer exposure times.

Figure 4.7 displays the minimum trace, real component of the complex permittivity for each time stamp in the 6-9 GHz bandwidth. There is an observable increase in $\varepsilon^{\prime}$ as the amount of degradation (cable age) increases. In Figure 4.8, the minimum $\varepsilon^{\prime}$ values at $7.5 \mathrm{GHz}$ are plotted versus exposure time to $140^{\circ} \mathrm{C}$.

Figure 4.9 shows the maximum trace, real component of the complex permittivity in the $6-9 \mathrm{GHz}$ bandwidth. This data also shows an increase in $\varepsilon^{\prime}$ as the degradation increases and also a larger variation between the $1152 \mathrm{hr}$ and $1224 \mathrm{hr}$ specimens. This could be due to the physical changes in the specimen as oxidation intensity nonlinearly increases with extended aging. The results for the maximum trace values for each degradation level are shown in Figure 4.10.

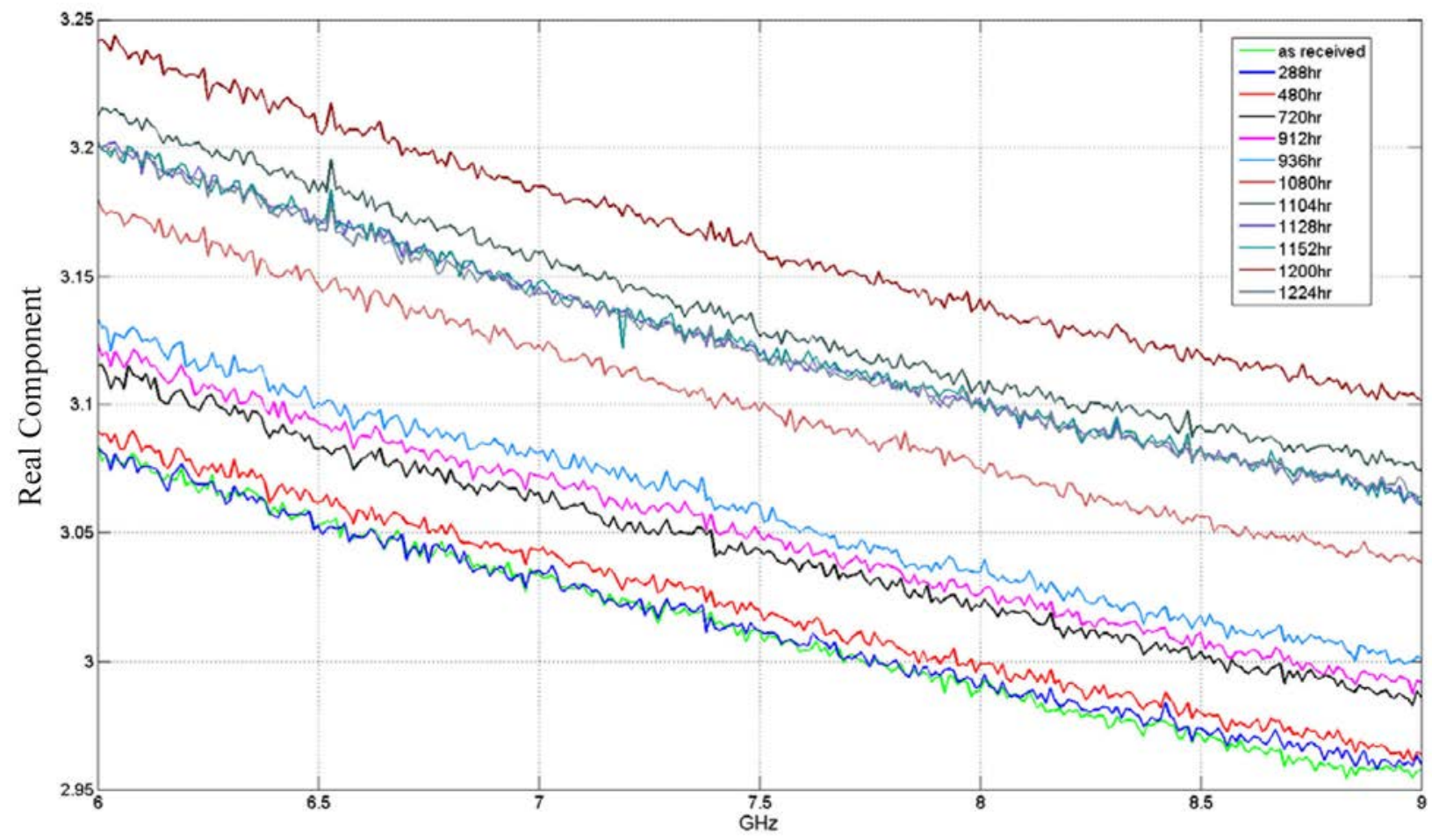

Figure 4.7. Minimum $\varepsilon^{\prime}$ Trace Values as a Function of Frequency and Cable Age 


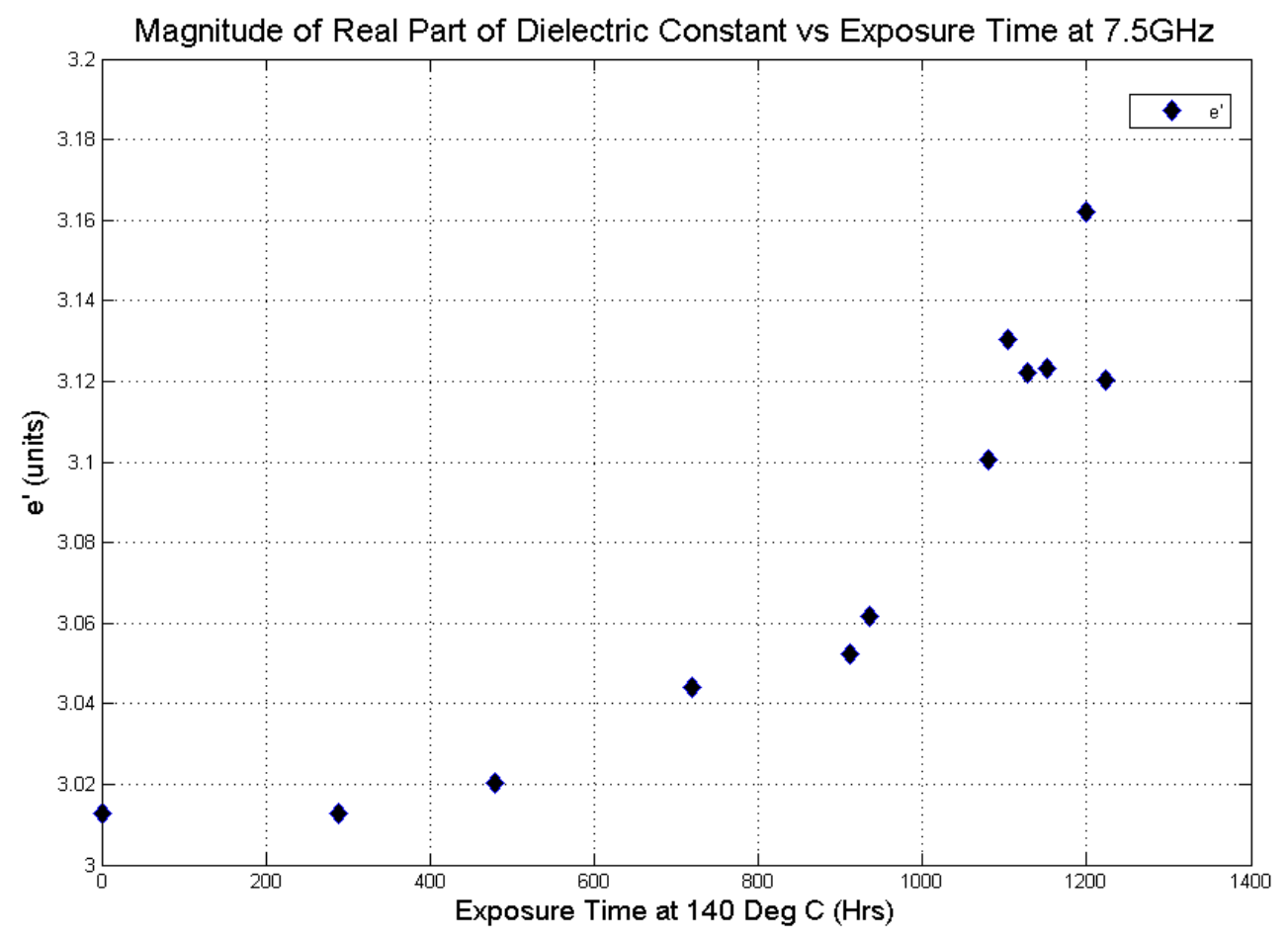

Figure 4.8. Minimum $\varepsilon^{\prime}$ Values at $7.5 \mathrm{GHz}$ vs. Cable Age

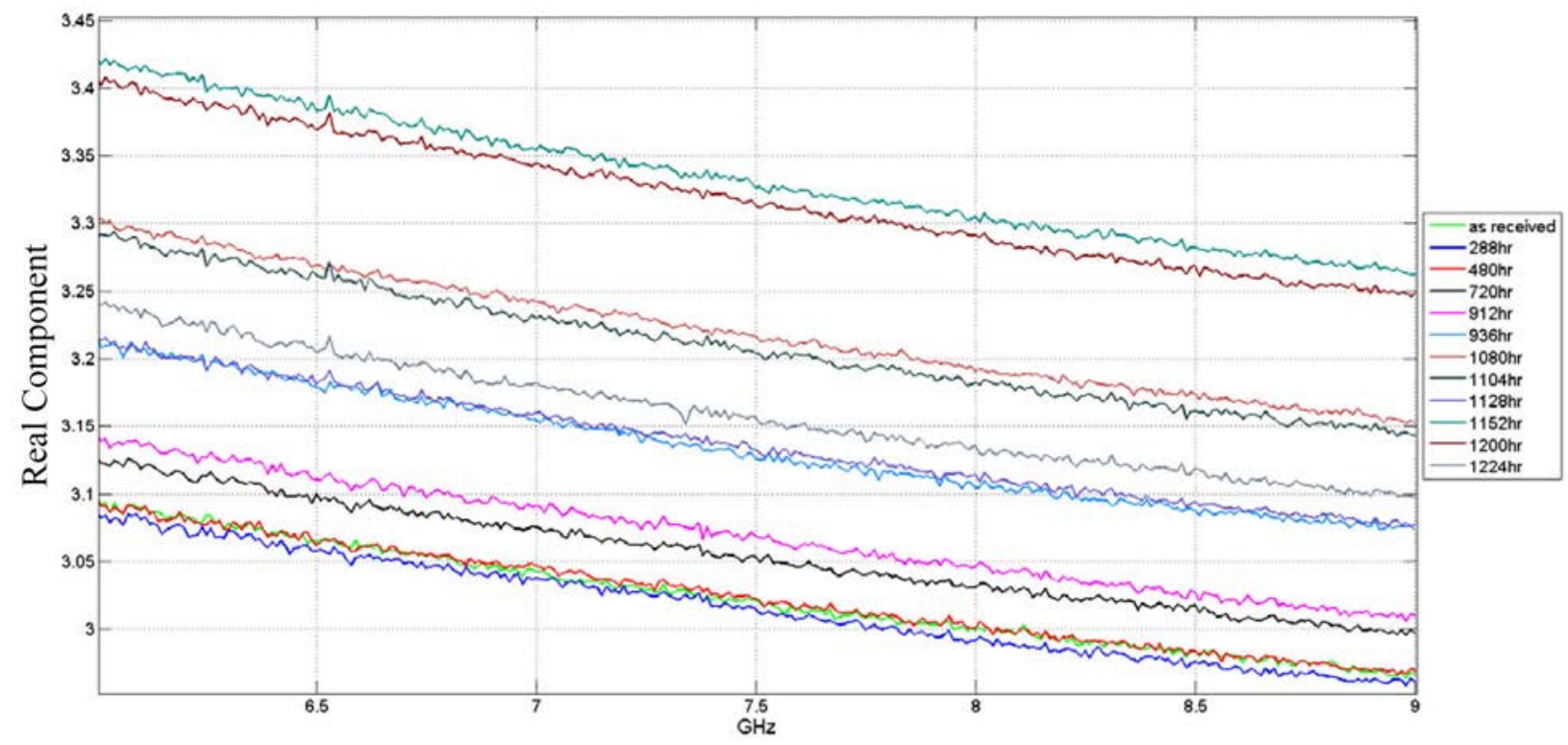

Figure 4.9. Maximum $\varepsilon^{\prime}$ Trace Values as a Function of Frequency and Cable Age 


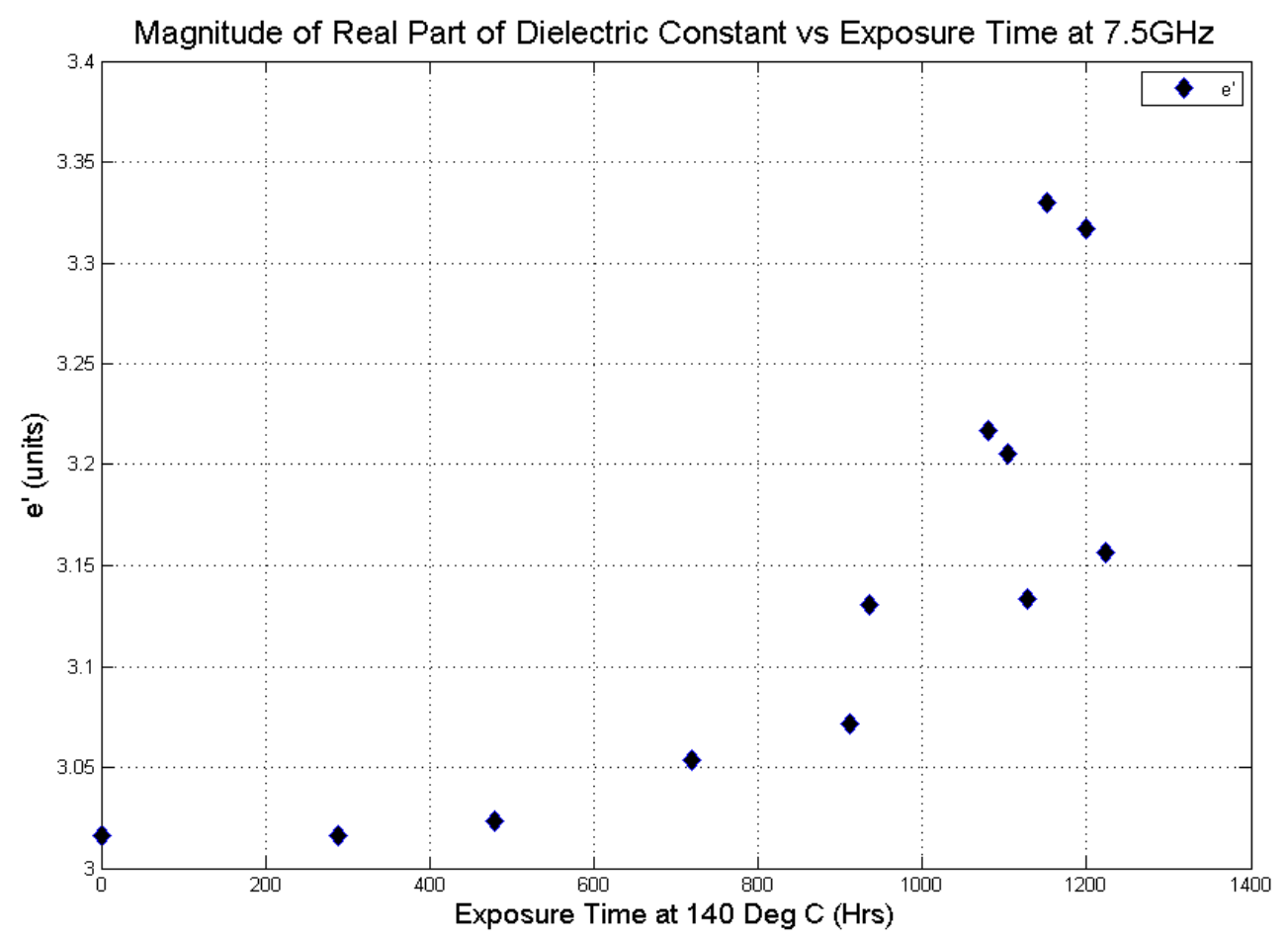

Figure 4.10. Maximum $\varepsilon^{\prime}$ Values at 7.5G Hz vs. Cable Age

\subsection{Discussion}

Several approaches to measuring key indicators of cable aging and degradation are be available, and include chemical, mechanical, and electrical measurements. The initial measurements (electrical) reinforce the assessment that measurements of dielectric permittivity (related to Tan $\delta$ ) are capable of providing information on cable age (and potentially remaining useful life, based on the relatively smooth increase of the real part of the dielectric constant with age). However, the measurements also indicate a certain level of sensitivity to a number of other factors, including the fixturing used for the probe as well as physical effects (surface damage) of the accelerated aging methodology.

In contrast, the acoustic measurement methods need further study to determine if the sound speed (and potentially attenuation) may be an indicator of age that directly correlates with indenter modulus and EAB. Additional studies may also be needed to determine the viability of approaches that directly measure changes in polymer chemistry as a result of aging; these are planned for future phases of research. 



\subsection{Summary}

Several approaches to nondestructively measuring key indicators of cable aging and degradation may are available, and include chemical, mechanical, and electrical measurements. The mechanical cable indenter method used for assessing cable age currently in the nuclear power industry is relatively simple; however, it provides a localized measure of cable age that may or may not be reflective of large sections of the cable. Electrical and acoustic measurements are potential alternative NDE approaches that may be capable of providing in-situ assessments of cable condition and remaining useful life.

The complex permittivity results using the high-temperature dielectric probe display promise for measuring a change in the real part of the dielectric constant and relating the change in this property to cable age. A total of 48 complex permittivity measurements were taken with cable insulation sample exposure times ranging from $0-1224$ hours at $140^{\circ} \mathrm{C}$. The real part of the dielectric constant was measured to have a 5-10\% increase over the span of exposure times.

Using acoustic methods that can provide a direct relation to the elastic modulus of the cable material seems feasible. Preliminary baseline velocity measurements in EPR polymers are in line with published data. However, the acoustic measurement methods need further study to determine if the sound speed (and potentially attenuation) may be an indicator of age that directly correlates with indenter modulus and EAB. Further work can be done to optimize the ultrasonic transducers selected and analysis methods as well as provide a more reliable method for positioning and coupling them. Additional studies may also be needed to determine the viability of approaches that directly measure changes in polymer chemistry as a result of aging; these are planned for future phases of research. 



\subsection{Path Forward}

Research going forward will focus on viability assessment of measurements of chemical, mechanical, and electrical key indicators that can provide data correlated to cable aging, and determine whether these diverse sets of measurements can provide synergistic information that can more effectively help in decisions on repair and replacement.

\subsection{Chemical Measurements}

\subsubsection{FTIR}

Infrared spectroscopy is an informative method for investigating chemical bond content arising from polymer degradation. Oxidative damage indicators, such as carbonyl content, will be monitored in cable polymer samples and correlated with polymer mechanical and other properties. Chemical changes may be spatially located through sample thickness by, for instance, measuring the content of polymer slices taken throughout a cable cross-section. In the figure below, an anvil attachment is pictured holding an EPR cross-section slice in a Diamond Smart iTR ${ }^{\mathrm{TM}}$ attenuated total reflectance (ATR) attachment of a Thermo Scientific FTIR instrument. ATR is a surface technique that allows FTIR spectra of the first several micrometers of a sample near the interface. The spectrum in the figure has clearly visible absorbance peaks corresponding to the ethylene-propylene polymer in an EPR specimen. Specimens that have experienced diffusion-limited oxidation (DLO) are expected to exhibit a graded profile of oxidation products through specimen thickness, which should be observable using ATR FTIR.

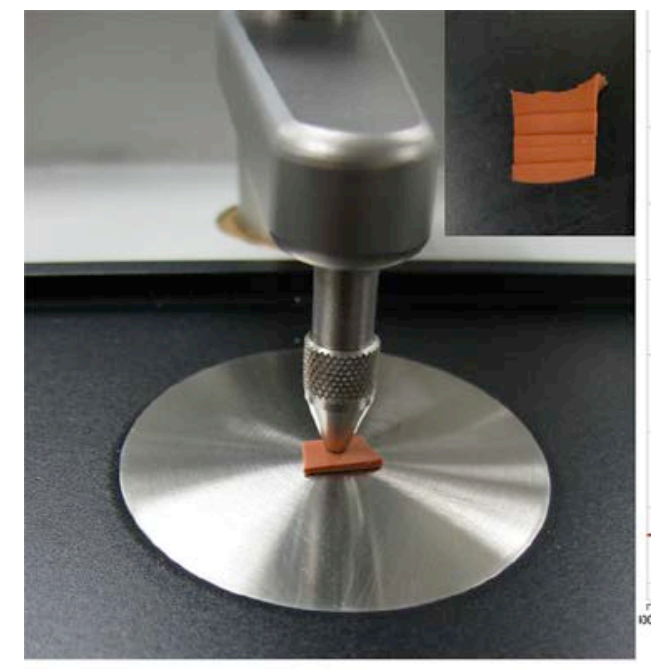

(a)

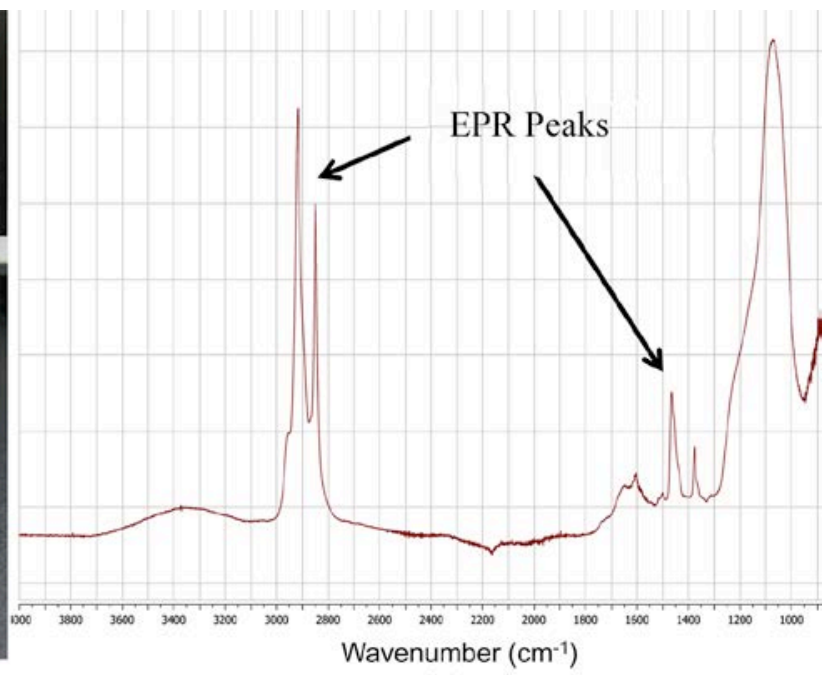

(b)

Figure 6.1. (a) Thermo Scientific Smart iTR ${ }^{\mathrm{TM}}$ Attenuated Total Reflectance Measurement of EPR Cross-section Slices. Inset: EPR cross-section sliced into layers. (b) FTIR ATR spectrum of EPR. 


\subsubsection{Differential Scanning Calorimeter}

DSC will be used as a monitor of change in composition of cable polymer content. Changes in molecular weight due to polymer chain scission and cross-linking will be reflected in changes in glass transition temperature. This data will be correlated with the sensitive change in glass transition measurable by dynamic mechanical analysis and to EAB. DSC will provide information on the effect of aging on the crystalline content of the cable polymer samples, which will also be correlated with mechanical properties and with density measurements.

\subsubsection{Thermogravimetric Analysis}

TGA investigation of insulation polymer material as a function of age will enable increased understanding of temperature stability effects of exposure damage. Increased oxygen content, chain scission, and cross-linking each result in changes to the mass loss profile with heating. This information will be correlated with changes in mechanical data and changes in other properties such as acoustic velocity and dielectric constant.

\subsection{Mechanical Measurements}

As the study progresses, additional mechanical measurement techniques will be evaluated. These evaluations can be directly correlated to existing relevant data acquired by the indenter-type nondestructive tests as well as the elongation-at-break data that have been evaluated both in the laboratory and in the field on existing power plants.

- DMA - Dynamic mechanical analysis will be further used in the investigation of moduli and glass transition changes in the polymer. The DMA modulus data can be used to assist the ultrasonic testing methods in determining the effects on bulk velocity and surface wave measurements.

- Ultrasonic Testing - The acoustic method used in the initial investigation will be further evaluated. This method required a known geometry, which is not readily available in a field measurement technique (i.e., wall thickness varies throughout every cable). Acoustic techniques investigated will use the cable geometry at the surface to provide bulk velocity and surface wave measurements. These data can then be compared to the DMA and other indenter type tests as well as to the breaking elongation data.

Development efforts for FY14 include optimization of a transducer (diameter, angle, frequency) and arrangement (location, size, coupling) for repeatable velocity and attenuation measurements on NPP cables. Appropriate mechanical fixtures will be developed to provide consistent application of ultrasonic energy to the cable specimens. In addition, the feasibility of measurement over longer segments of cables will be explored through the application of guided acoustic wave approaches.

\subsection{Electrical Measurements}

As the study progresses additional electrical measurement methods will be investigated. These methods will be assessed on the test apparatus and evaluated as possible methods for further exploration. Some of the methods currently being considered are: 
Electrical Permittivity - The electrical permittivity measurements and Tan $\delta$ measurements that were initially investigated will be further explored using a more refined technical approach that removes ambiguities caused by material thickness constraints relative to probe diameter and pressure. Key to this effort will be the fabrication of mechanical fixtures that apply a fixed calibrated pressure between the high-temperature dielectric probe and sample under test.

Complex permittivity measurements performed in FY13 demonstrated the ability to measure a change in the real part of the dielectric constant of the cable insulation in relation to cable aging. Considerations for this measurement technique are: repeatability of data, physical geometry of the cable and the sample size requirements of the dielectric probe, calibrated repeatable pressure applied for each sample set, the frequency band used to isolate and monitor the change in the real part of the dielectric constant, and whether this type of measurement can be performed remotely and in real-time as the cable is being exposed to temperatures representative of the cable aging.

Development efforts for FY14 include studies with additional specimens of aged EPR and XLPE cables to quantify the effects of aging on permittivity and insulation resistance. Mechanical fixtures will be developed to limit the effect of probe coupling and positioning on the measurement. The data sets will be used in future phases to develop models that may be used for remaining life calculations. In parallel to the efforts on quantifying changes in electrical properties, initial work will begin on examining the field deployability of these techniques, and whether similar assessments may be made inductively, using electromagnetic coupling through the insulation.

\subsection{Testing Apparatus}

PNNL is developing a test setup that can age the cable while cable property measurements are performed. The concept is to supply an operating voltage and current to an electrical cable that is representative of a cable used in a nuclear power plant. In the test setup, a medium-voltage EPR cable is run through a high-temperature furnace. The cable remains in the furnace for the duration of testing and measurements can be made on the cable exterior to the furnace. Figure 6.2 provides a graphic representation of the test apparatus.

System development is underway, and Figure 6.3 provides a photograph of the test apparatus that is almost complete.

A load can be placed on the cable (in our case, a motor operating at $120 \mathrm{VAC}$ ). The duration of the test is expected to be in the range of 1 to 3 months. The circuit is being monitored from a LabVIEW integrated control system, which allows measurements to be taken continuously at specified intervals. Additional test points are located in each junction box to allow for additional (more precise) instrumentation to be added. Additionally, a rack that can hold up to 12 cut samples allows for simultaneous aging of smaller samples for other nondestructive or destructive testing. 


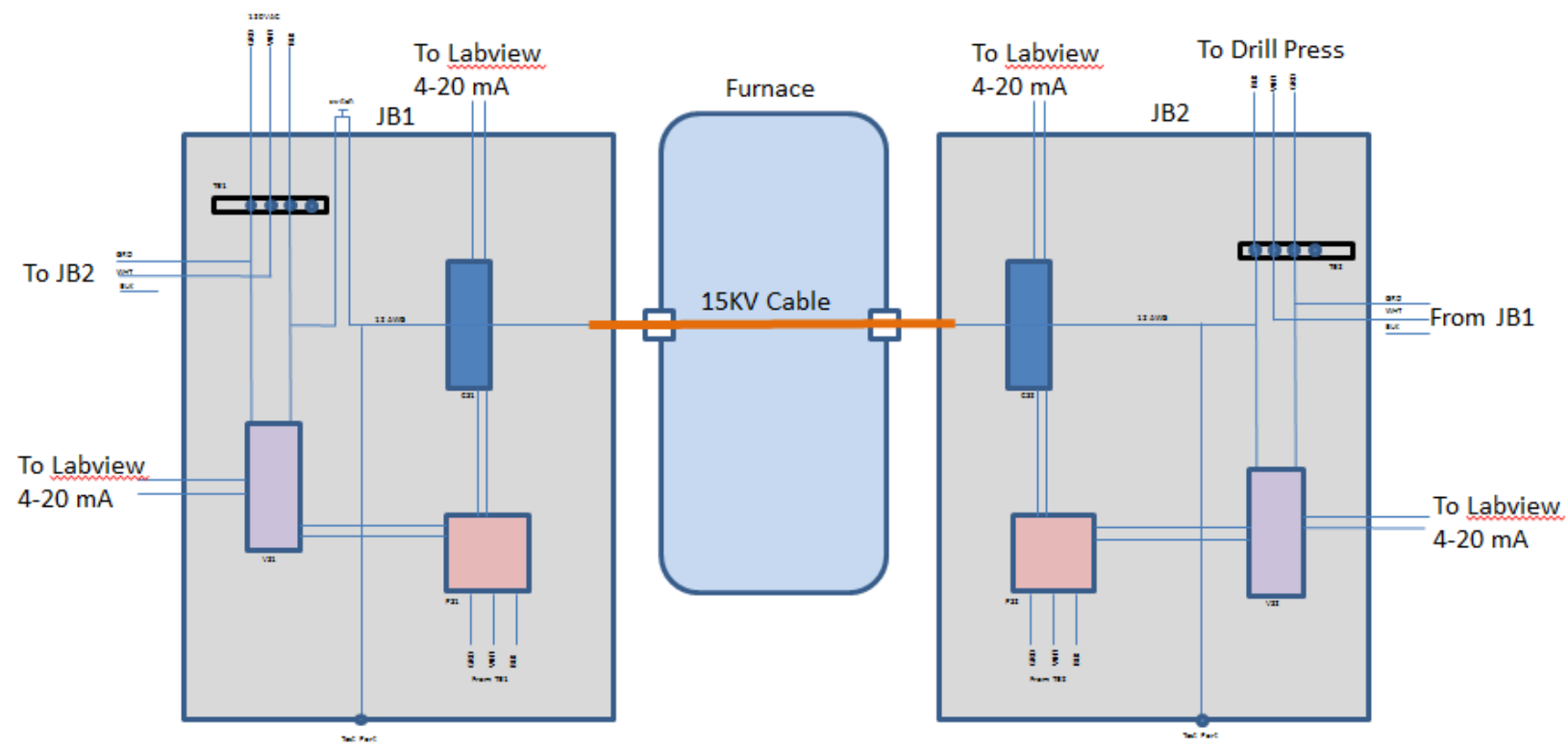

Figure 6.2. Graphic Representation of Measurement Circuit (where JB - Junction Box, TB - Terminal Block, CS - Current Sensor, VS - Voltage Sensor, PS - Power Supply)

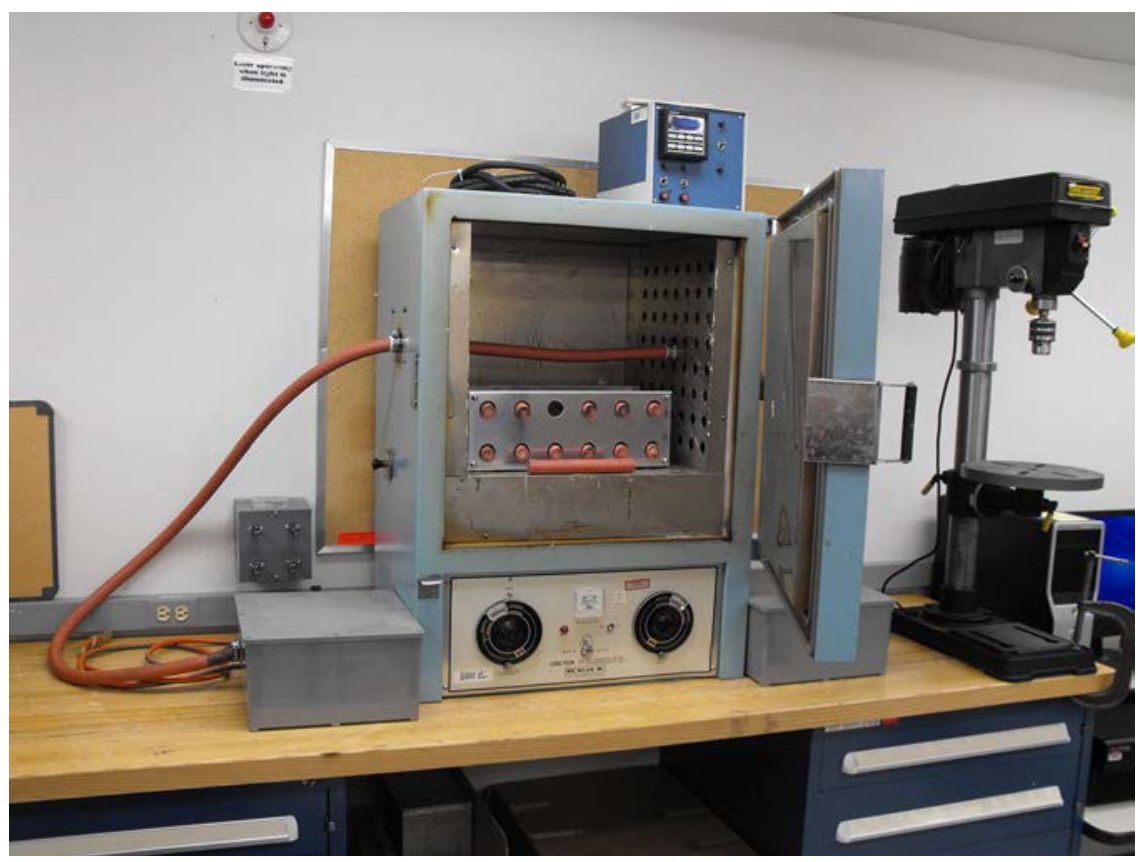

Figure 6.3. Cable Aging Test Apparatus 


\subsection{References}

Crawford RJ. 1998. Plastics Engineering, 3rd Edition. Elsevier.

Crine JP. 2005. "Influence of Electro-Mechanical Stress on Electrical Properties of Dielectric Polymers." IEEE Transactions on Dielectrics and Electrical Insulation 12(4):791-800.

Ensminger D and LJ Bond. 2011. Ultrasonics: Fundamentals, Technology and Applications, Third Edition (Revised and Expanded). CRC Press, Boca Raton, Florida.

EPRI. 1994. Low-Voltage Environmentally-Qualified Cable License Renewal Industry Report; Revision 1. TR-103841, Electric Power Research Institute (EPRI), Palo Alto, California.

EPRI. 2005. Initial Acceptance Criteria Concepts and Data for Assessing Longevity of Low-Voltage Cable Insulations and Jackets. TR-1008211, Electric Power Research Institute (EPRI), Palo Alto, California.

Gillen KT, RA Assink and R Bernstein. 2005. Nuclear Energy Plant Optimization (NEPO) Final Report on Aging and Condition Monitoring of Low-Voltage Cable Materials. SAND2005-7331, Sandia National Laboratories, Albuquerque, New Mexico.

Gillen KT, M Celina and RL Clough. 1999. "Density Measurements as a Condition Monitoring Approach for Following the Aging of Nuclear Power Plant Cable Materials." Radiation Physics and Chemistry 56(4):429-447.

Grellmann W and S Seidler, Eds. 2007. Polymer Testing. Hanser Publications, Cincinnati, Ohio.

IAEA. 2012. Assessing and Managing Cable Ageing in Nuclear Power Plants. IAEA Nuclear Energy Series No. NP-T-3.6, International Atomic Energy Agency (IAEA), Vienna.

Ikehara J, T Ikeda, T Ashida and M Fujii. 1998. "Degradation Diagnosis of Polymeric Materials Using Ultrasonic Waves." In Proceedings of the 1998 Ultrasonics Symposium, pp. 1667-1170, Vol. 2. October 5-8, 1998, Sendai, Japan. DOI 10.1109/ULTSYM.1998.765046. Institute of Electrical and Electronics Engineers Inc., Piscataway, New Jersey.

Krautkrämer J and H Krautkrämer. 1990. Ultrasonic Testing of Materials, 4th fully revised Edition. Springer-Verlag, New York.

Lofaro R, E Grove, M Villaran, P Soo and F Hsu. 2001. Assessment of Environmental Qualification Practices and Condition Monitoring Techniques for Low-Voltage Electric Cables: Condition Monitoring Test Results. NUREG/CR-6704, Volume 2, U.S. Nuclear Regulatory Commission, Washington, D.C.

Pao Y-H. 1983. "Elastic Waves in Solids." Journal of Applied Mechanics 50(4b):1152-1164.

Roberts RA. 1990. "Model of the Acoustic Microscope Response to Scattering by a Near-surface Void." Journal of Nondestructive Evaluation 9(2-3):181-196.

Scheirs J. 2000. Compositional and Failure Analysis of Polymers: A Practical Approach. John Wiley \& Sons, Ltd. 
Sepe M. 1998. Dynamic Mechanical Analysis for Plastics Engineering (PDL Handbook Series). Plastics Design Library, Norwich, New York.

Simmons KL, P Ramuhalli, DL Brenchley and JB Coble. 2012. Light Water Reactor Sustainability (LWRS) Program - Non-Destructive Evaluation (NDE) R\&D Roadmap for Determining Remaining Useful Life of Aging Cables in Nuclear Power Plants. PNNL-21731, Pacific Northwest National Laboratory, Richland, Washington.

Vanderbilt. 2010. Vanderbilt Rubber Handbook, 14th Edition. R. T. Vanderbilt Co. Inc., Norwalk, Connecticut.

Yamamoto T and T Minakawa. 2009. The Final Report of The Project of 'Assessment of Cable Aging for Nuclear Power Plants'. JNES-SS-0903, Incorporated Administrative Agency Japan Nuclear Energy Safety Organization, Nuclear Energy System Safety Division, Tokyo, Japan. 


\section{Appendix A}

Real Component of the Complex Permittivity Measurements for Aged Cable Samples, $200 \mathrm{MHz}-20 \mathrm{GHz}$ 



\section{Appendix A}

\section{Real Component of the Complex Permittivity Measurements for Aged Cable Samples, $200 \mathrm{MHz}-20 \mathrm{GHz}$}

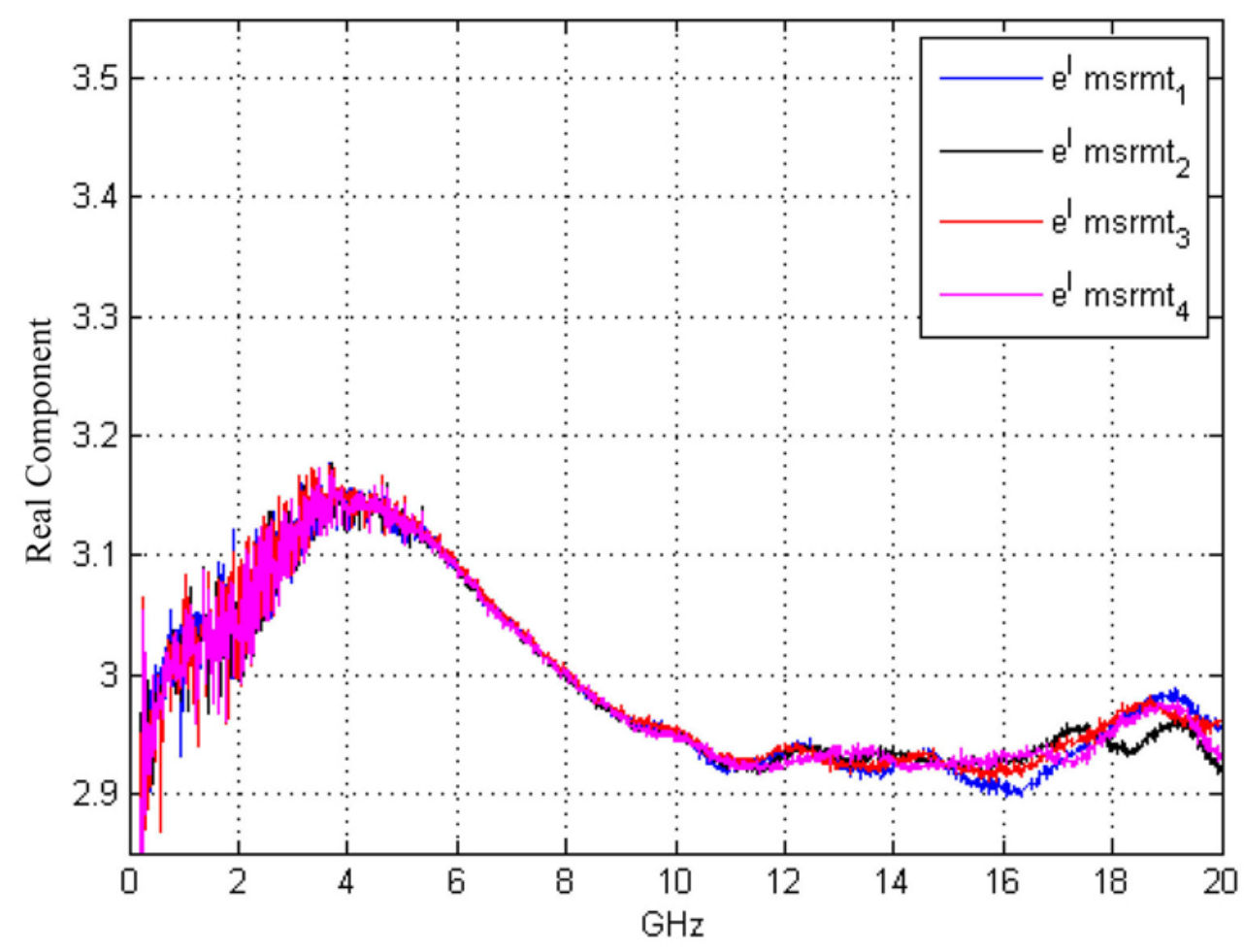

Figure A.1. Sample As-Received, Real Component of the Complex Permittivity 


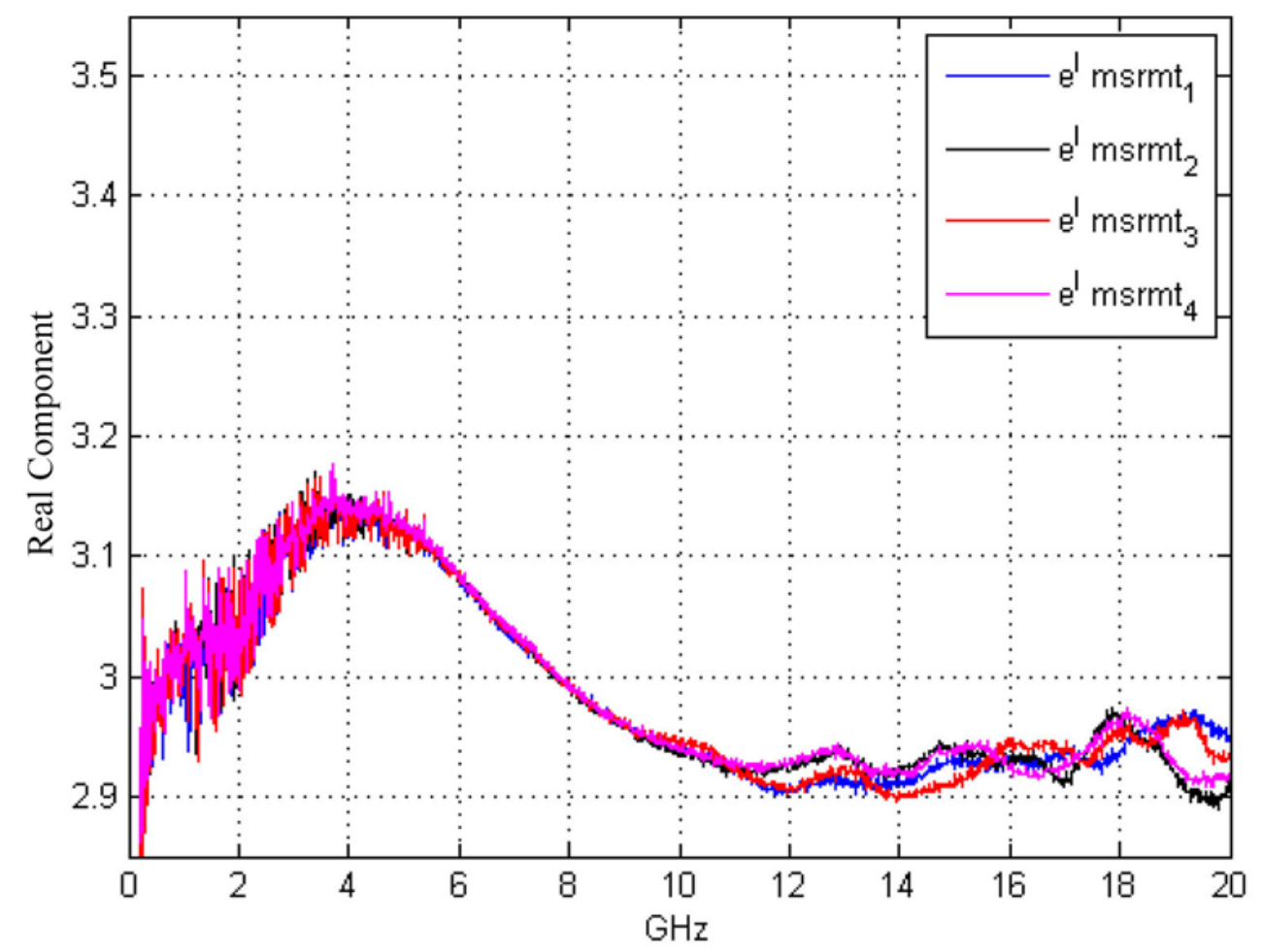

Figure A.2. Sample Exposed to $140^{\circ} \mathrm{C}$ for 288 Hours, Real Component of the Complex Permittivity

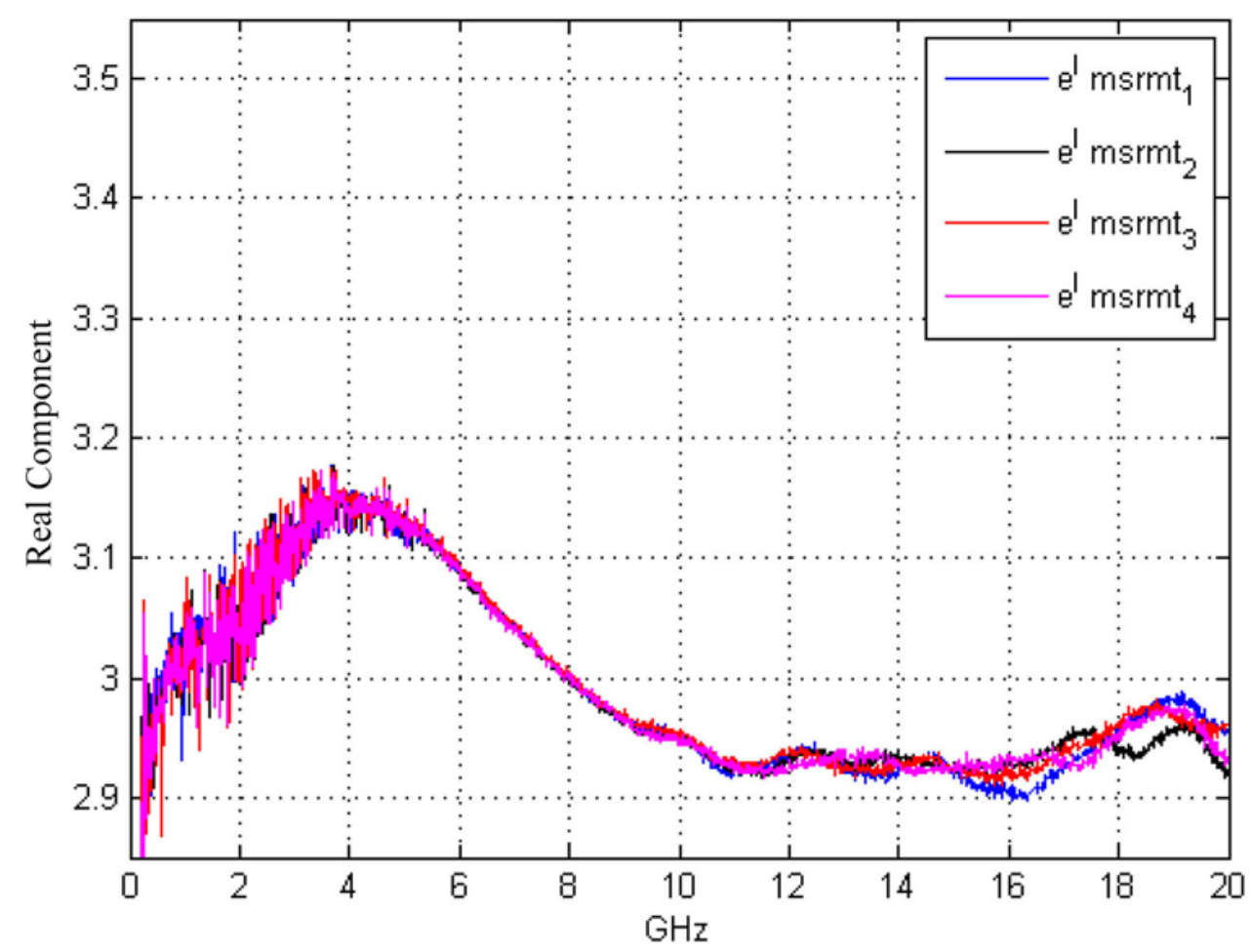

Figure A.3. Sample Exposed to $140^{\circ} \mathrm{C}$ for 480 Hours, Real Component of the Complex Permittivity 


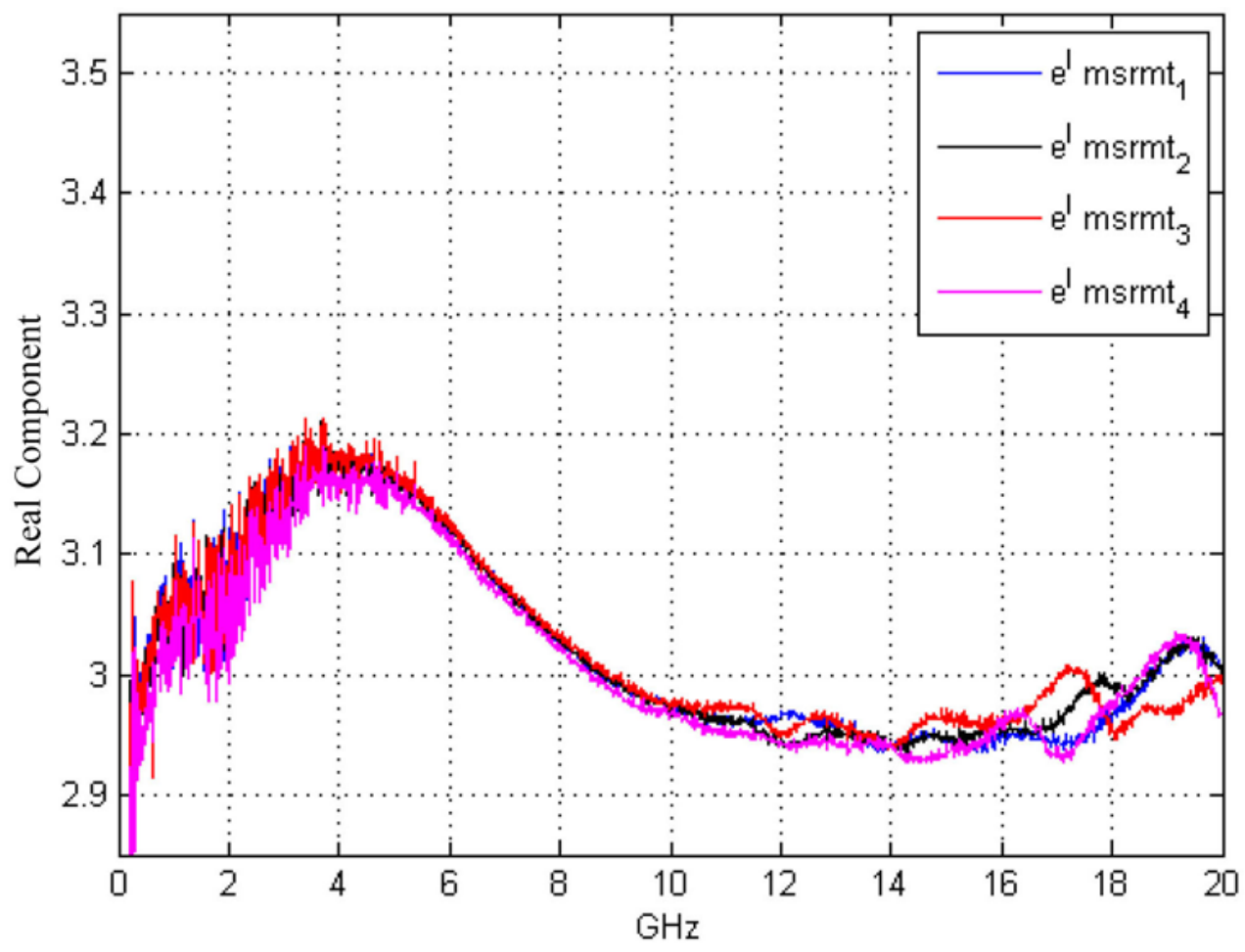

Figure A.4. Sample Exposed to $140^{\circ} \mathrm{C}$ for 720 Hours, Real Component of the Complex Permittivity

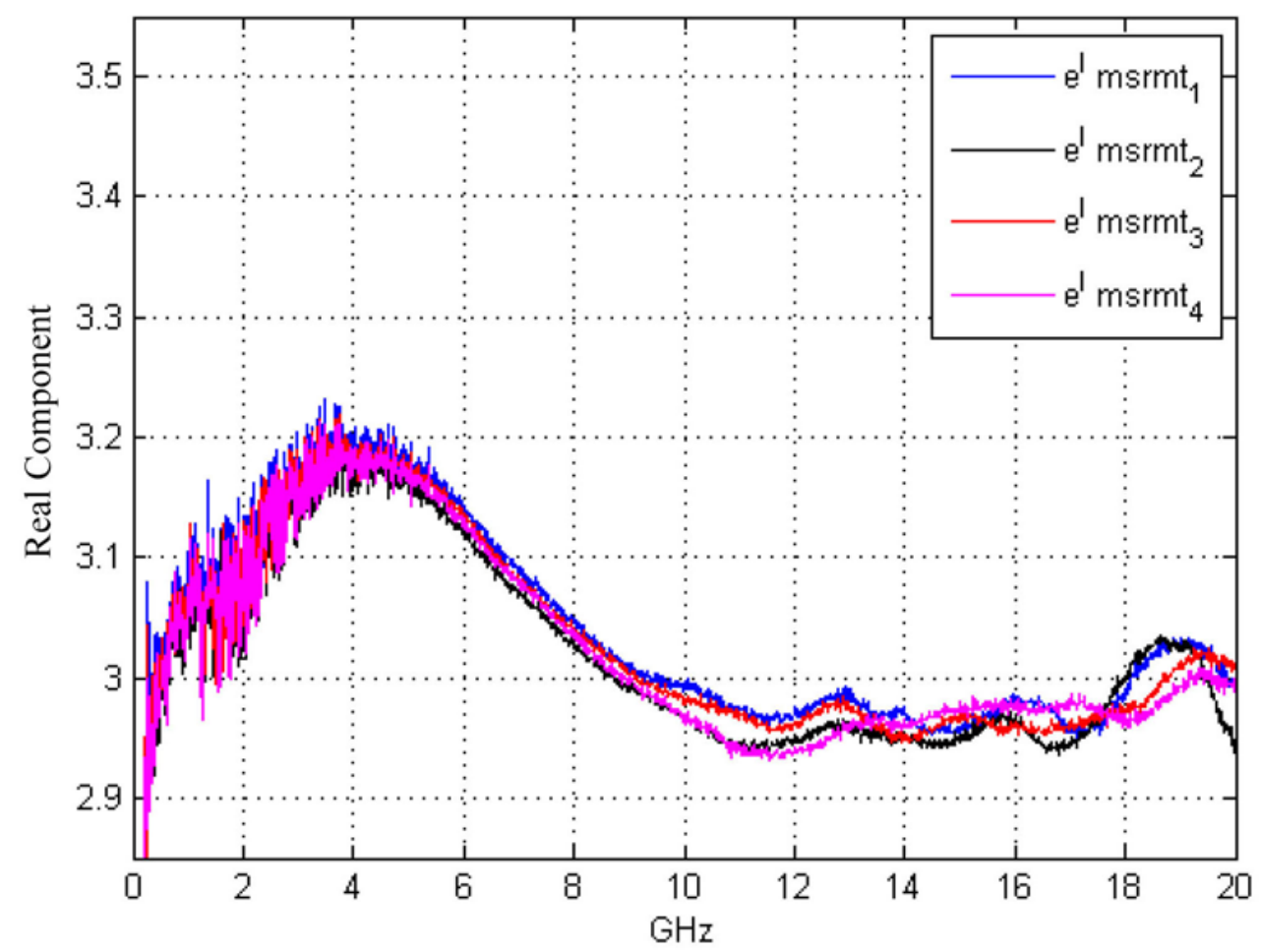

Figure A.5. Sample Exposed to $140^{\circ} \mathrm{C}$ for 912 Hours, Real Component of the Complex Permittivity 


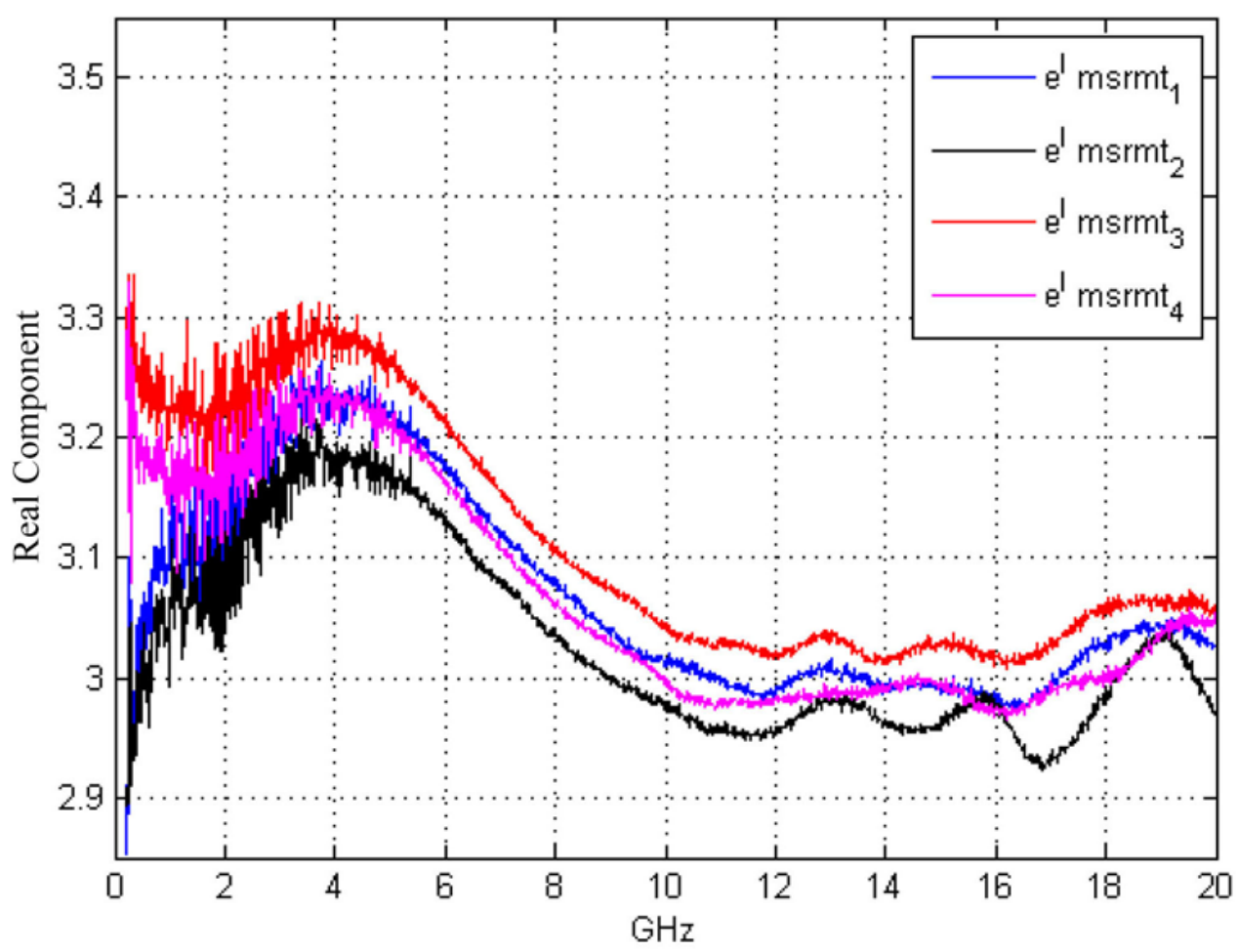

Figure A.6. Sample Exposed to $140^{\circ} \mathrm{C}$ for 936 Hours, Real Component of the Complex Permittivity

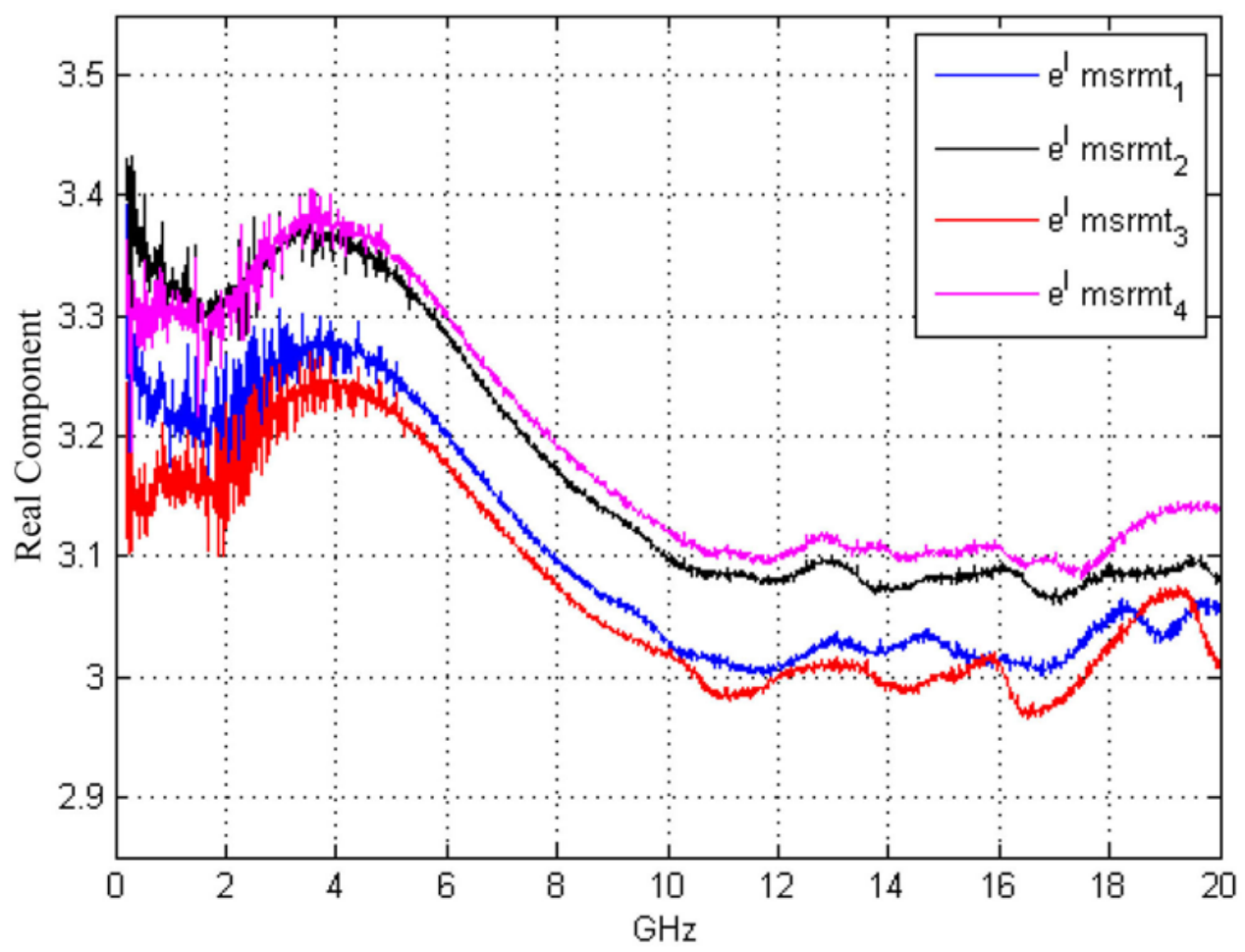

Figure A.7. Sample Exposed to $140^{\circ} \mathrm{C}$ for 1080 Hours, Real Component of the Complex Permittivity 


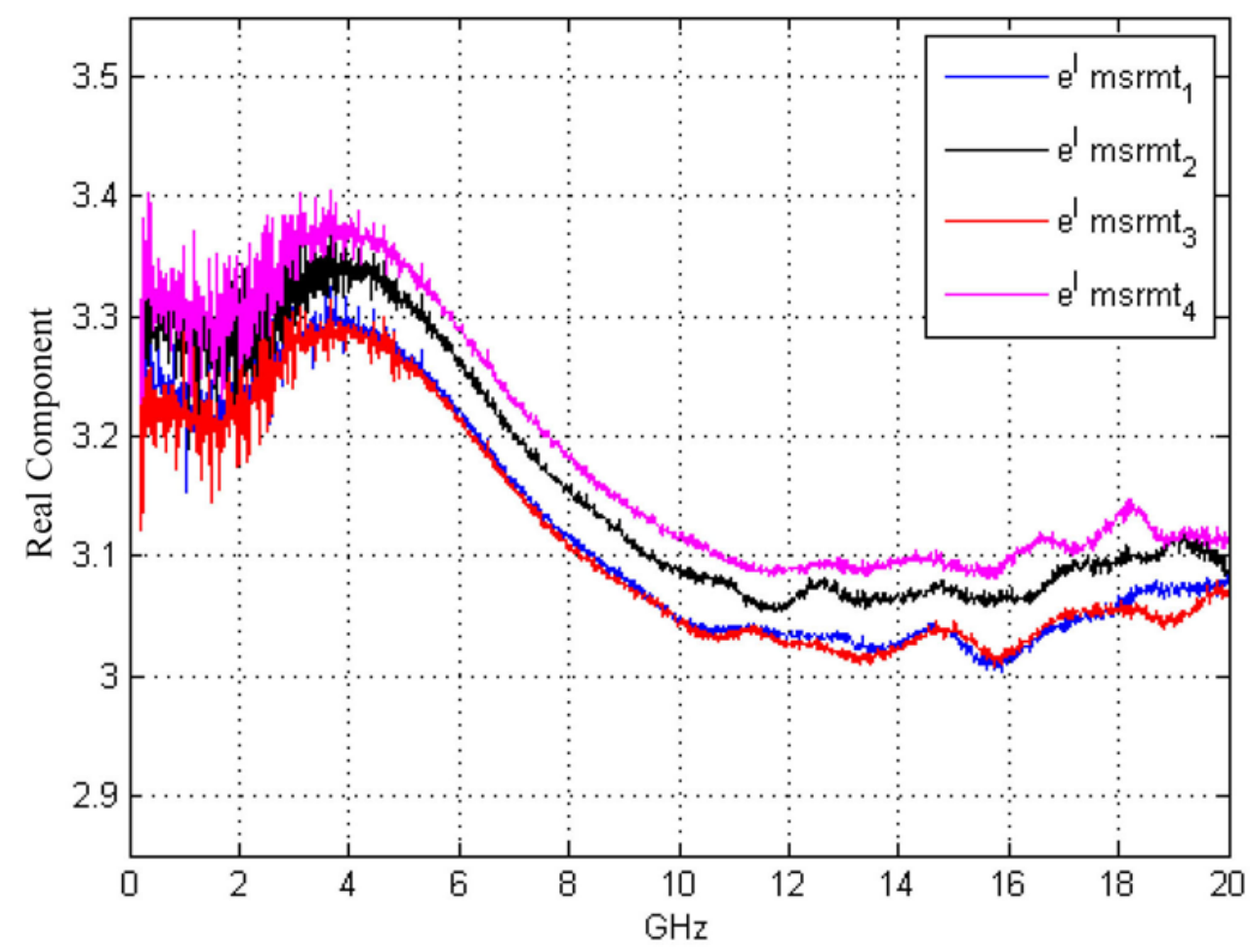

Figure A.8. Sample Exposed to $140^{\circ} \mathrm{C}$ for 1104 Hours, Real Component of the Complex Permittivity

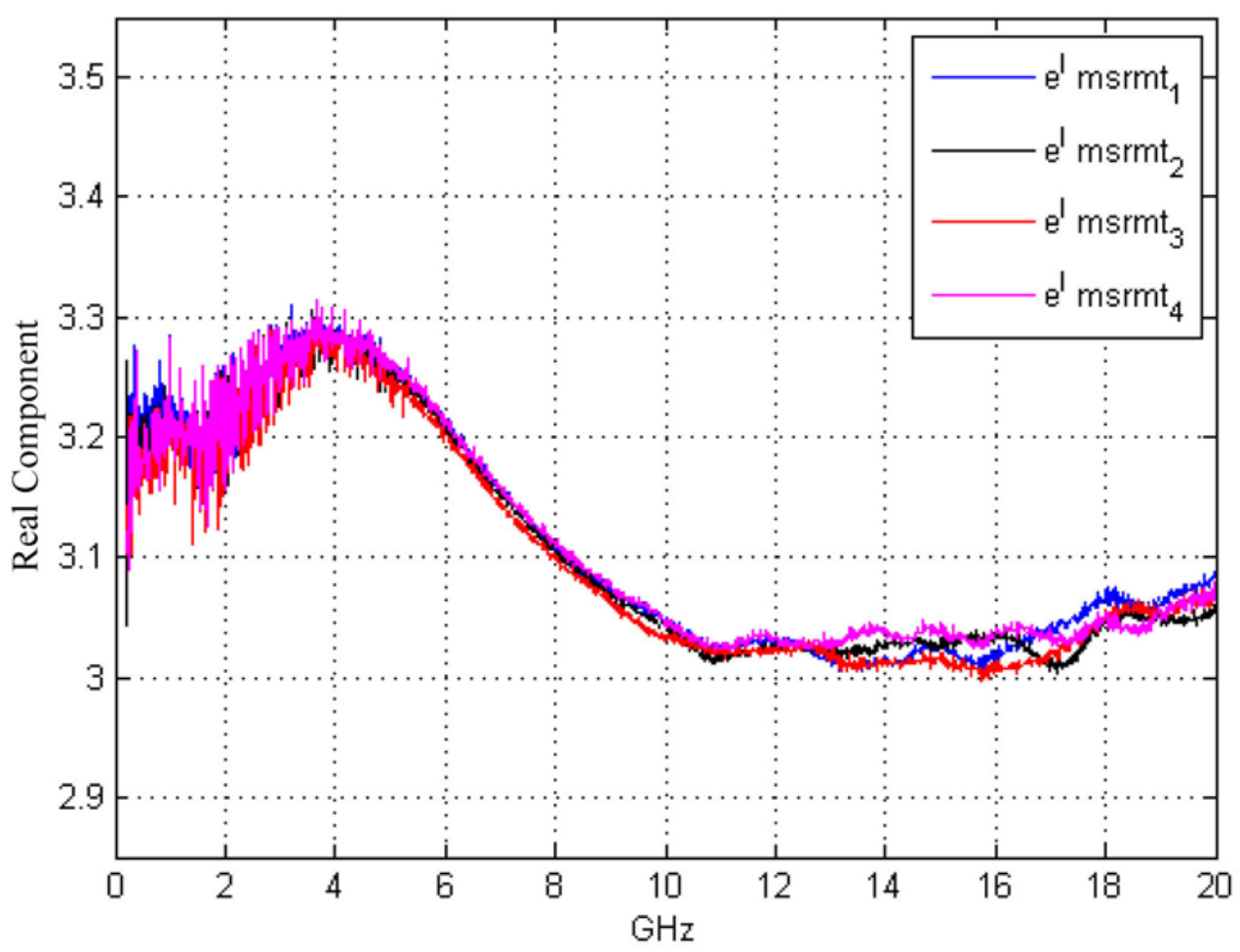

Figure A.9. Sample Exposed to $140^{\circ} \mathrm{C}$ for 1128 Hours, Real Component of the Complex Permittivity 


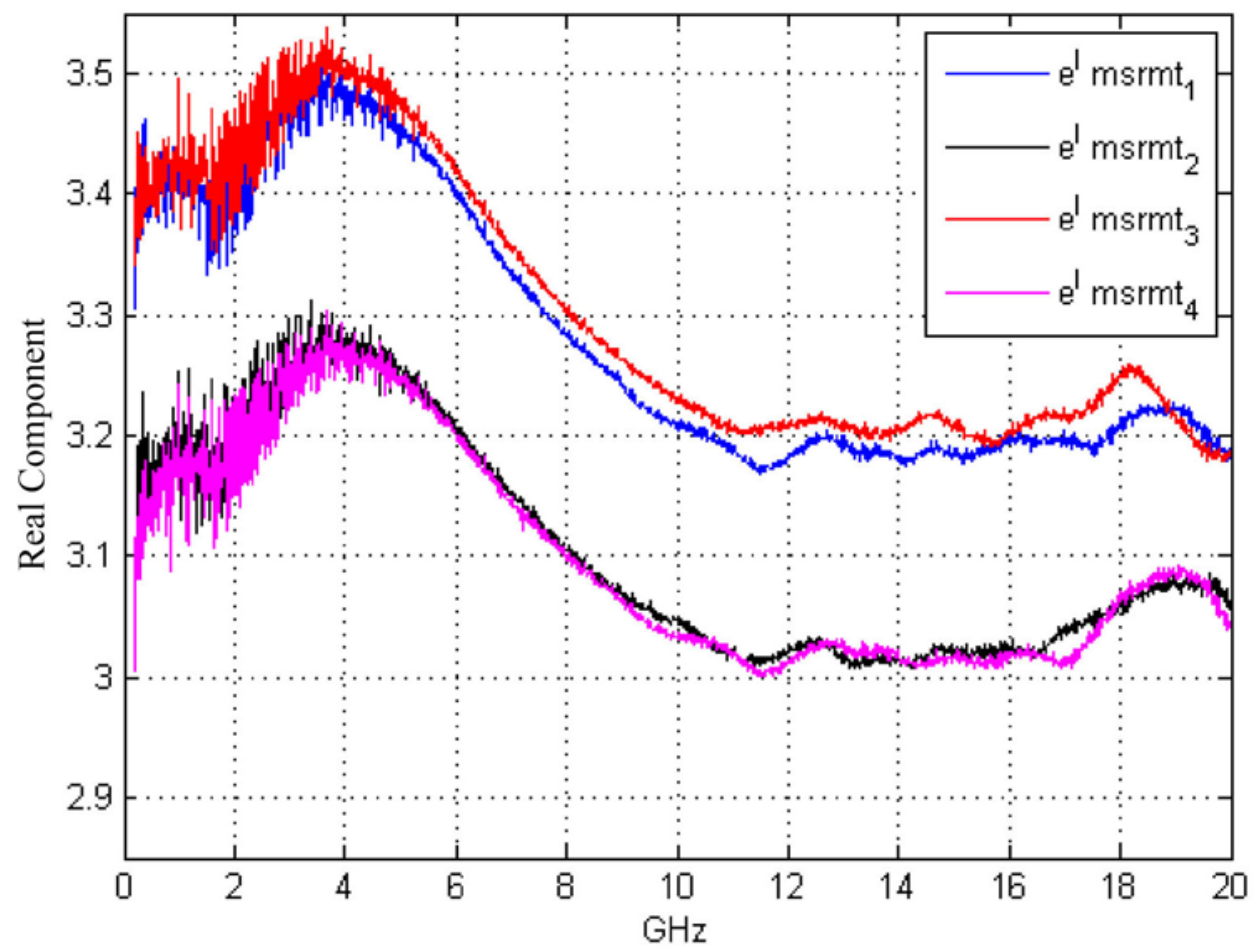

Figure A.10. Sample Exposed to $140^{\circ} \mathrm{C}$ for 1152 Hours, Real Component of the Complex Permittivity

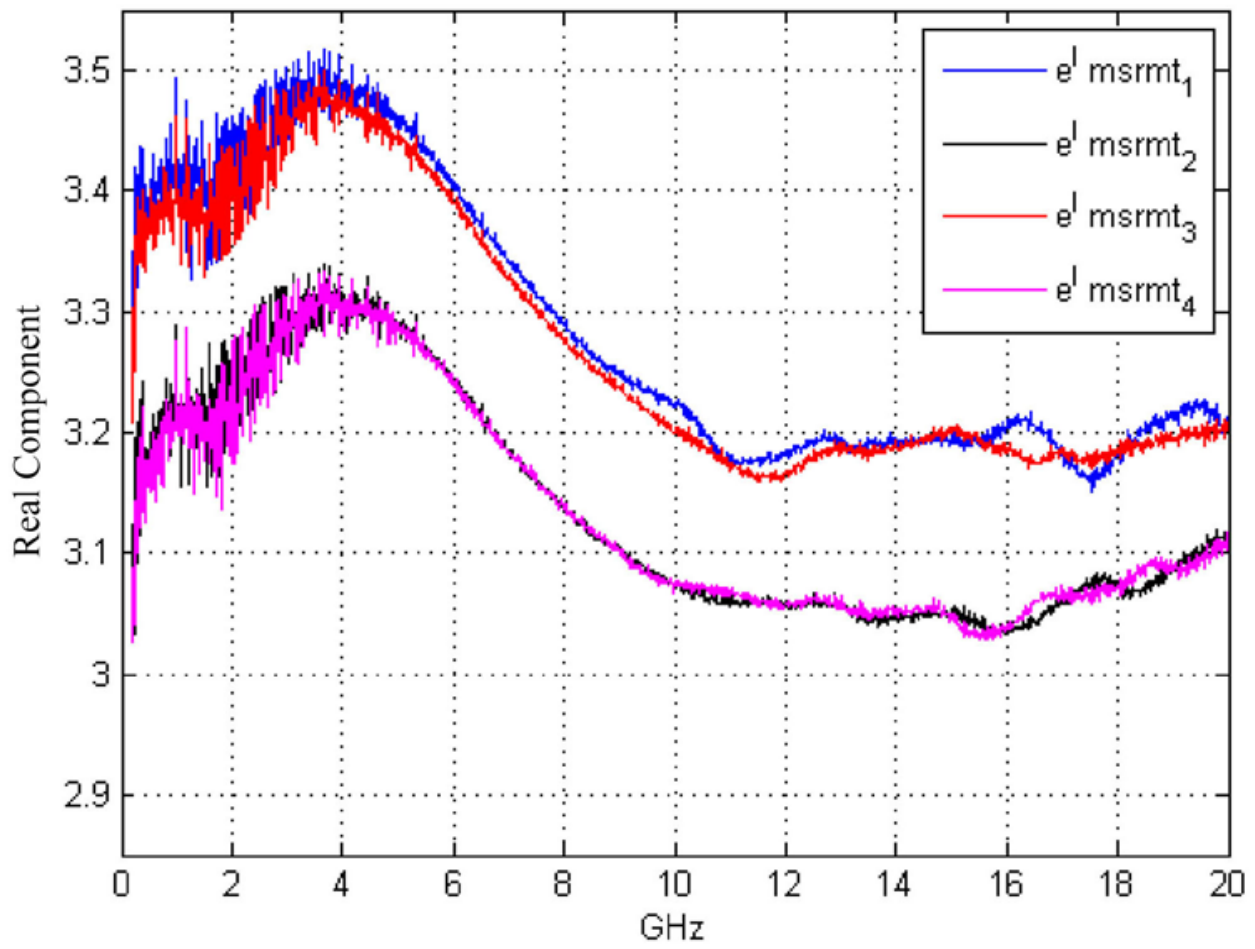

Figure A.11. Sample Exposed to $140^{\circ} \mathrm{C}$ for 1200 Hours, Real Component of the Complex Permittivity 



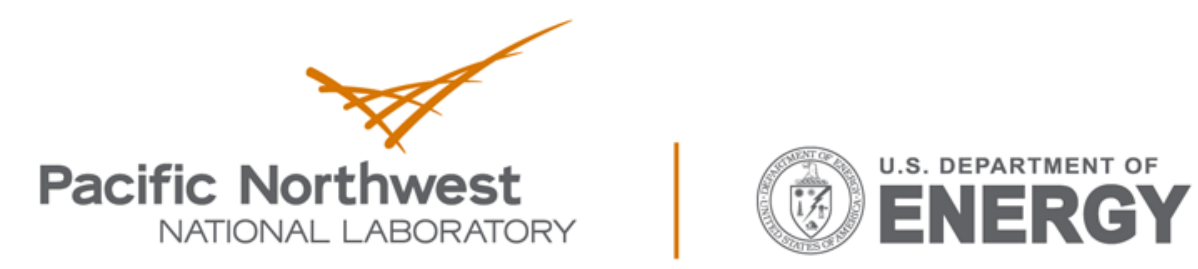

Proudly Operated by Battelle Since 1965

902 Battelle Boulevard

P.O. Box 999

Richland, WA 99352

1-888-375-PNNL (7665)

www.pnnl.gov 